\title{
LACUNAS FOR HYPERBOLIC DIFFERENTIAL OPERATORS WITH CONSTANT COEFFICIENTS I \\ BY
}

\author{
M. F. ATIYAH, R. BOTT, L: GÅRDING
}

\section{Introduction}

Ивану Георгиевичу Петровскому

с уважением и восхищением

The theory of lacunas for hyperbolic differential operators was created by I. G. Petrovsky who published the basic paper of the subject in $1945\left({ }^{1}\right)$. Although its results are very clear, the paper is difficult reading and has so far not lead to studies of the same scope. We shall clarify and generalize Petrovsky's theory.

The various kinds of linear free wave propagation that occur in the mathematical models of classical physics are of the following general type. There is an elastic $(n-1)$ dimensional medium whose deviation from rest position is described by a function $u(x)$ with values in some $R^{N}$ and defined in some open subset $O$ of $R^{n}$, one of the coordinates being time. When there are no exterior forces, $u$ satisfies a system of $N$ linear partial differential equations $P(x, \partial / \partial x) u(x)=0$ where $P$ is a linear partial differential operator with smooth matrix-valued coefficients. Further, a unit impulse applied at some point $y$ produces close to $y$ a movement that propagates with a locally bounded velocity in all directions. This movement is smooth outside a system of possibly criss-crossing wave fronts and vanishes outside the cone of propagation $K(P, y)$, a conical region with its vertex at $y$ and bounded by the fastest fronts. Mathematically, the movement is described by a distribution $E=E(P, x, y)$ which is defined when $x$ is close to $y$, vanishes when $x$ is outside $K(P, y)$ and satisfies

$$
P(x, \partial / \partial x) E(P, x, y)=\delta(x-y)
$$

so that $E$ is a (right) fundamental solution of $P$. Under these circumstances we say that $P$ is a hyperbolic operator. Briefly, $P$ is hyperbolic if it has a fundamental solution with

(1) See the references. 
conical support as described above. When $P=P(\partial / \partial x)$ has constant coefficients, its fundamental solution $E$ is defined for all $x, y$, it is unique and depends only on the difference $x-y$. It will then be denoted by $E(P, x)$ and we have

$$
P(\partial / \partial x) E(P, x)=E(P, x) P(\partial / \partial x)=\delta(x) .
$$

The support of $E$ is contained in a closed cone with its vertex at the origin which is proper in the sense that, apart from its vertex, it is contained in an open half-space $x \vartheta=x_{1} \vartheta_{1}+$ $\ldots+x_{n} \vartheta_{n}>0$ where $0 \neq \vartheta \in R^{n}$. We let hyp $p_{N}(\vartheta)$ be the class of these operators and write it as hyp $(\vartheta)$ when $N=1$ so that the operators are scalar. An easy argument (Lemma 3.2) shows that $P \in \operatorname{hyp}_{N}(\vartheta)$ if and only if $\operatorname{det} P \in$ hyp $(\vartheta)$. The scalar operators are basic. Let hyp $(\vartheta, m)$ be all $P \in$ hyp $(\vartheta)$ of order $m$ and let Hyp $(\vartheta)$ and Hyp $(\vartheta, m)$ be the homogeneous operators in hyp $(\vartheta)$ and hyp $(\vartheta, m)$ respectively. The operators $P$ in hyp $(\vartheta)$ can be characterized algebraically. Writing $P=P(D)$ where $D=\partial / i \partial x$ is the imaginary gradient, we attach to $P$ and its principal part $a$ their characteristic polynomials $P(\xi)$ and $a(\xi), \xi \in \mathbf{C}^{n}$. Then $P \in$ hyp $(\vartheta)$ if and only if $a(\vartheta) \neq 0$ and $P(\xi+t \vartheta) \neq 0$ for all real $\xi$ when $|\operatorname{Im} t|$ is large enough. The elements of hyp $(\vartheta)$ will also be considered as polynomials with this property. It follows that hyp $(\vartheta)=$ hyp $(-\vartheta)$. When $P=a \in \operatorname{Hyp}(\vartheta, m)$, the condition means that the equation $a(\xi+t \vartheta)=0$ has $m$ real roots $t$ for every real $\xi$. In particular, apart from a complex constant factor, such an $a(\xi)$ is real. If the roots are all different except when $\xi$ is proportional to $\vartheta, a$ is said to be strongly hyperbolic and the corresponding class of operators is denoted by $\operatorname{Hyp}^{0}(\vartheta)$. When $P \in$ hyp $(\vartheta)$ then $a \in$ Hyp $(\vartheta)$ but if $a \in \operatorname{Hyp}(\vartheta)$ then every $P$ with principal part $a$ is in hyp $(\vartheta)$ only if $a \in \mathrm{Hyp}^{0}(\vartheta)$. The class of $P$ with $a$ in $\mathrm{Hyp}^{0}(\vartheta)$ will be denoted by $\operatorname{hyp}^{0}(\vartheta)$ and its elements are also called strongly hyperbolic. A scalar operator $P(x, \partial / \partial x)$ with variable coefficients turns out to be hyperbolic if the operators $x \rightarrow P(y, \partial / \partial x)$ are strongly hyperbolic and their order is constant. We shall only treat operators with constant coefficients and, apart from some occasional remarks, also restrict ourselves to scalar operators. The determinants of the non-scalar hyperbolic operators (systems) that occur in mathematical physics are as a rule not strongly hyperbolic.

When $P \in$ hyp $(\vartheta)$, write $P=a+b$ where $a \in$ Hyp $(\vartheta)$ is the principal part of $P$ so that the degree of $b$ is less than that of $P$. It turns out that $b$ cannot depend on more variables than $a$. More precisely, if $Q(\xi), \xi \in Z=\mathbf{C}^{n}$, is a polynomial, let $L(Q)$, the lineality of $Q$, be the maximal linear space $L$ such that $Q$ is a polynomial on the quotient $Z / L$. When $L(Q)=0$, $Q$ is said to be complete. When $P \in$ hyp $(\vartheta), P$ and $a$ have the same lineality $L(P)=L(a)$. Its codimension $n(P)=n(a)$ is said to be the reduced dimension of $P$ and $a$.

The class hyp $(\vartheta, 0)$ consists of all non-vanishing constants. For them, $L(P)=Z$. The class hyp $(\vartheta, 1)$ consists of all $P$ of degree 1 such that $a(\vartheta) \neq 0$ and $a(\xi)$ is real apart 
from a constant factor. In that case $L(P)$ is the hyperplane $a(\xi)=0$ and has dimension $n-1$. With a suitable choice of coordinates, every complete $a \in \operatorname{Hyp}(\vartheta, 2), n \geqslant 2$, is a multiple of the wave polynomial $\Delta(\xi)=\xi_{1}^{2}-\xi_{2}^{2}-\ldots-\xi_{n}^{2}$ and then $\Delta(\vartheta)>0$. The corresponding operator $\Delta=\Delta(\partial / \partial x)$ is the wave operator. It is strongly hyperbolic. When $n=2$, every $a \in \mathrm{Hyp}(\vartheta)$ is a product of real linear factors such that $a(\vartheta) \neq 0$. When these factors are all different, $a \in \operatorname{Hyp}^{0}(\vartheta)$.

By the algebraic definition of hyperbolicity, the classes hyp $(\vartheta)$ and Hyp $(\vartheta)$ are closed under multiplication and factors of hyperbolic polynomials are hyperbolic. The process of localization also leads to hyperbolic polynomials. When $P(\xi)$ is a polynomial of degree $m$, let us develop $t^{m} P\left(t^{-1} \xi+\zeta\right)$ in ascending powers of $t$ and let $t^{p} P_{\xi}(\zeta)$ be the first nonvanishing term. The integer $p=m_{\xi}(P)$ is called the multiplicity of $\xi$ relative to $P$ and the polynomial $\zeta \rightarrow P_{\xi}(\zeta)$ the localization of $P$ at $\xi$. When $P=a$ is homogeneous, $a(\xi+t \zeta)=$ $t^{p} a_{\xi}(\zeta)+$ higher terms and $p=m_{\xi}(a)$ is simply the degree of the polynomial $\zeta \rightarrow a_{\xi}(\zeta)$. It turns out that if $P \in$ hyp $(\vartheta)$ and $\xi$ is real, then $P_{\xi} \in$ hyp $(\vartheta)$ and $a_{\xi} \in$ Hyp $(\vartheta)$ is the principal part of $P_{\xi}$. In particular, $m_{\xi}(P)=m_{\xi}(a)$. When $P \in$ hyp $^{0}(\vartheta)$ is strongly hyperbolic, all $P_{\xi}$ with $\xi \neq 0$ are constant or first degree polynomials so that, accordingly, $n\left(P_{\xi}\right)=n\left(a_{\xi}\right)=0$ or 1 .

When $a(\xi)$ is a homogeneous polynomial, let $A: a(\xi)=0$ be the associated hypersurface in $Z$ and let $\operatorname{Re} A$ be the real part of $A$. The lineality $L(a)$ depends only on $A$ and will also be denoted by $L(A)$. In particular, when $x=\left(x_{1}, \ldots, x_{n}\right)$ belongs to the dual complex space $Z$, let $X: x_{1} \xi_{1}+\ldots x_{n} \xi_{n}=0$ be the associated hyperplane and $\operatorname{Re} X$ its real part. By the algebraic definition of hyperbolicity, when $a \in \operatorname{Hyp}(\vartheta, m)$ the surface $\operatorname{Re} A$ intersects every real straight line with direction $\vartheta$ in $m$ real points. When $a \in \operatorname{Hyp}^{0}(\vartheta, m)$ and the line misses the origin, they are all different. The component $\Gamma(A, \vartheta)$ of $\operatorname{Re} Z-\operatorname{Re} A$ that contains $\vartheta$ is an open convex wedge whose edge is $\operatorname{Re} L(A)$. When $P \in$ hyp $(\vartheta)$ has principal part $a$, we also put $\Gamma(P, \vartheta)=\Gamma(A, \vartheta)$.

A basic property of hyperbolic polynomials is that $P \in$ hyp $(\vartheta)$ implies $P \in$ hyp $(\eta)$ for every $\eta \in \Gamma(P, \vartheta)=\Gamma(A, \vartheta)$. More precisely, there is a convex open subset $\Gamma_{1}=\Gamma_{1}(A, \vartheta)$ of $\Gamma=\Gamma(A, \vartheta)$ such that $s \Gamma_{1} \subset \Gamma_{1}$ when $s \geqslant 1$ and $\Gamma=\bigcup s \Gamma_{1}, s>0$, and $P(\xi \pm i \eta) \neq 0$ when $\eta \in \Gamma_{1}$. The fundamental solution $E(P, x)=E(P, \vartheta, x)$ of $P$ with support in $x \vartheta \geqslant 0$ is simply an inverse Fourier-Laplace transform of $P^{-1}$,

$$
E(P, \vartheta, x)=(2 \pi)^{-n} \int P(\xi-i \eta)^{-1} e^{i x(\xi-i \eta)} d \xi, \quad \eta \in \Gamma_{1} .
$$

Here the right side is independent of $\eta$ and, by the Paley-Wiener-Schwartz theorem, $E$ vanishes at $x$ unless $x \eta \geqslant 0$ for all $\eta \in \Gamma$. This condition defines the dual cone $K=K(P, \vartheta)=$ 
$K(A, \vartheta)$ of $\Gamma$. This dual cone is the propagation cone for $P$ relative to $\vartheta$. It is closed and convex and meets every half-space $x \eta \leqslant$ const, $\eta \in \Gamma$, in a compact set. It is also the closed convex hull of the support of $E$. It is contained in and spans the orthogonal complement of $\operatorname{Re} L(A)$ whose dimension is the reduced dimension $n(a)=n(P)$. More precisely, put $\xi=\left(\xi^{\prime}, \xi^{\prime \prime}\right)$ where $\xi^{\prime}, \xi^{\prime \prime}$ are coordinates in $Z / L(A)$ and $L(A)$ and let $x^{\prime}, x^{\prime \prime}$ be the dual coordinates in $Z^{\prime}$. Then $P^{\prime}\left(\xi^{\prime}\right)=P(\xi)$ is complete, $P(D)=P^{\prime}\left(D^{\prime}\right) \otimes 1$ where 1 is the identity operator on $L(A)$, and $(1)$ shows that $E(P, \vartheta, x)=E\left(P^{\prime}, \vartheta^{\prime}, x^{\prime}\right) \otimes \delta\left(x^{\prime \prime}\right)$. This reduces the study of fundamental solutions to those of complete operators. Putting $P=a+b$ we also have

$$
E(P, \vartheta, x)=\sum_{0}^{\infty}(-1)^{k} b(D)^{k} E\left(a^{k+1}, \vartheta, x\right)
$$

so that there is a further reduction to the homogeneous case. (2) follows from (1) and the fact that $b a^{-1}(\xi-i s \vartheta)$ tends to zero uniformly in $\xi$ when $s \rightarrow \infty$ (Leif Svensson (1969)).

There is a simple connection between the fundamental solution $E(x)=E(P, \vartheta, x)$ of $P \in$ hyp $(\vartheta)$ and the fundamental solution $E_{\xi}(x)=E\left(P_{\xi}, \vartheta, x\right)$ of a localization of $P$ pointed out to us by L. Hörmander. If $p=m_{\xi}(P)$ is the multiplicity of $\xi \in \operatorname{Re} Z$ and $t \rightarrow \infty$, then $t^{m-p} e^{i t x \xi} E(x)$ tends to $E_{\xi}(x)$ in the distribution sense. As a consequence we have the important fact that

$$
0 \neq \xi \in \operatorname{Re} Z \Rightarrow \mathrm{S} E_{\xi} \subset \mathrm{SS} E,
$$

where S denotes support and SS singular support.

Let us now consider the local cones $\Gamma_{\xi}=\Gamma\left(P_{\xi}, \vartheta\right)=\Gamma\left(A_{\xi}, \vartheta\right)$ and local propagation cones $K_{\xi}=K\left(P_{\xi}, \vartheta\right)=K\left(A_{\xi}, \vartheta\right)$ where $a_{\xi}$ is the localization of the principal part of $P$. It is easy to show that $\Gamma_{\xi} \supset \Gamma(A, \vartheta), K_{\xi} \subset K(A, \vartheta)$ with equality if and only if $\xi \in \operatorname{Re} L(A)$. When $a(\xi) \neq 0, \Gamma_{\xi}$ is the whole space and $K_{\xi}=0$. When $a(\xi)=0$ but $\operatorname{grad} a(\xi) \neq 0$, then $\Gamma_{\xi}$ is a half-space and $K_{\xi}$ is a half-ray normal to $\operatorname{Re} A$. When $a$ is strongly hyperbolic, these exhaust all possiblities. At singular points of $\operatorname{Re} A$ outside the origin, $\Gamma_{\xi}$ may be smaller than a half-space and $K_{\xi}$ bigger than a half-ray. The local cones have important continuity properties. The functions $\xi, a \rightarrow \Gamma\left(A_{\xi}, \vartheta\right)$ and $\xi, a \rightarrow K\left(A_{\xi}, \vartheta\right)$ are inner and outer continuous respectively. Here inner (outer) continuity of a map $\sigma$ from a space $\{\tau\}$ to subsets of another space means that $\sigma\left(\tau^{\prime}\right) \cap \sigma(\tau),\left(\sigma\left(\tau^{\prime}\right) \cup \sigma(\tau)\right)$, is close to $\sigma(\tau)$ when $\tau^{\prime}$ is close to $\tau$.

Let ${ }^{0} \operatorname{Re} A$ be the real dual of the hypersurface $\operatorname{Re} A$, i.e. the set of $x \in \operatorname{Re} Z^{\prime}$ such that $\operatorname{Re} X$ is tangent to $\operatorname{Re} A$. It has been known for a long time, in particular through the work of Herglotz (1926-28) that if $a \in \mathrm{Hyp}(\vartheta), E=E(a, \vartheta, x)$ is holomorphic in $\stackrel{\circ}{K}(A, \vartheta)$ outside ${ }^{0} \operatorname{Re} A$ in significant special cases. For strongly hyperbolic $a$, the proof is due to Petrovsky. There is also no better result. In fact, if $a \in \operatorname{Hyp}^{0}(\vartheta)$, then, by (3), the singular support of 
$E$ contains the support of every $E_{\xi}$ with $\xi \neq 0$. Since the corresponding $a_{\xi}$ is constant or linear this support is all of $K_{\xi}=K\left(A_{\xi}, \vartheta\right)$. Now $K(A, \vartheta) \cap{ }^{0} \operatorname{Re} A$ is the union of these $K_{\xi}$ and this shows that $E$ is nowhere smooth across ${ }^{0} \operatorname{Re} A$ in $K(A, \vartheta)$. The same proof works, also in the inhomogeneous case, when $\operatorname{Re} A$ is non-singular outside the origin. When $\operatorname{Re} A$ is singular, ${ }^{0} \operatorname{Re} A$ is not the proper object to consider and we replace it by the wave front surface $W=W(A, \vartheta)$ defined as the union of all $K\left(A_{\xi}, \vartheta\right)$ for $0 \neq \xi$ real. If $a$ is not complete, $W=K=K(A, \vartheta)$ but apart from that case, $W$ has codimension 1 , is contained in ${ }^{\circ} \operatorname{Re} A \cap K$ and contains the boundary of $K$. Unlike ${ }^{\circ} \operatorname{Re} A, W$ is an outer continuous function of $a \in \operatorname{Hyp}(\vartheta)$. It also has the stronger continuity property that there are elements $b$ in $\operatorname{Hyp}^{0}(\vartheta, m)$ arbitrarily close to a given $a$ such that $W(B, \vartheta)$ meets a given conical neighbourhood of a given ray in $W(A, \vartheta)$.

We shall show that $E(P, \vartheta, \cdot)$ is holomorphic outside $W(P, \vartheta)=W(A, \vartheta)$ when $P \in$ hyp $(\vartheta)$ and $a$ is the principal part of $P$. When $P=a$, the proof is achieved by a shift of the chain of integration in (1). The new chains are images of maps $\xi \rightarrow \xi-i v(\xi)$ where $v(\xi)$ is a smooth vector field such that $v(\xi) \in \Gamma_{\xi}(A, \vartheta)$ for all $\xi$. By the inner continuity of the function $\xi \rightarrow \Gamma_{\xi}$, such fields exist and we show that small multiples of $v$ have the additional property that $a(\xi-i s v(\xi)) \neq 0$ when $0<s<1$. By Cauchy's theorem, the constant vector field $v_{0}(\xi)=\eta$ in (1) can be replaced by any such $v$ homotopic to $v_{0}$ provided the exponential stays bounded. When $x \bar{\epsilon} \pm W(A, \vartheta)$, we can in fact find a $v$ with these properties such that, in addition, $a(\xi-i v(\xi))^{-1}$ is bounded and $x v(\xi) \leqslant-\varepsilon(\xi)$ for some $\varepsilon>0$ and all large $\xi$. But then we have absolute convergence in (1) and the integral is a holomorphic function close to $x$. Applying this result and some simple estimates to (2) takes care of the inhomogeneous case.

When $P=a \in \operatorname{Hyp}(\vartheta, m)$ is homogeneous, we can use the homogeneity to perform a radial integration in (1). We perform this operation using our modified form of (1). The vector fields that are adapted to this process constitute a family $V=V(A, X, \vartheta)$ characterized by the properties that $v(\xi) \in C^{\infty}$ for $0 \neq \xi$ real, $v(\lambda \xi)=|\lambda| v(\xi)$ when $0 \neq \lambda \in R$, $v(\xi) \in \Gamma\left(A_{\xi}, \vartheta\right) \cap \operatorname{Re} X$ for all $\xi \neq 0, a(\xi-i s v(\xi)) \neq 0$ for all real $\xi \neq 0$ and $0<s \leqslant 1$. When $x \bar{\epsilon} \pm W(A, \vartheta)$, this family is not empty and any two elements of it are homotopic. Modulo constant factors the result (Theorem 7.16) may be presented as follows.

If $a \in \operatorname{Hyp}(\vartheta, m)$ and $x \in K(A, \vartheta)-W(A, \vartheta)$, then

$$
\begin{aligned}
& (\partial / \partial x)^{\nu} E(a, \vartheta, x) \sim \int_{\alpha^{*}}(x \xi)^{a} \xi^{\nu} a(\xi)^{-1} \omega(\xi), \quad q \geqslant 0, \\
& (\partial / \partial x)^{\nu} E(a, \vartheta, x) \sim \int_{t_{x} \partial \alpha^{*}}(x \xi)^{a} \xi^{\nu} a(\xi)^{-1} \omega(\xi), \quad q<0 .
\end{aligned}
$$

8-702909 Acta mathematica. 124. Imprimé le 8 Avril 1970. 
Here $(\partial / \partial x)^{\nu}=\left(\partial / \partial x_{1}\right)^{\nu_{1}} \ldots, \xi^{\nu}=\xi_{1}^{\nu_{1}} \ldots, \omega(\xi)=\Sigma(-1)^{j-1} \xi_{j} d \xi_{1} \ldots \widehat{d \xi}_{j} \ldots d \xi_{n}$ and (4) and (5) hold when $q=m-n-|\nu| \geqslant 0$ and $q<0$ respectively, $|\nu|=v_{1}+\ldots+v_{n}$. The integrands are rational $(n-1)$-forms of homogeneity zero on $Z$ and hence also closed forms of maximal degree in $(n-1)$-dimensional projective space $Z^{*}=\dot{Z} / \dot{\mathbf{C}}$ where a dot indicates that the origin is removed. They are holomorphic on $Z^{*}-A^{*}$ and $Z^{*}-A^{*} \cup X^{*}$ respectively. Here, if $B$ is a part of $Z, B^{*}$ denotes the image of $\dot{B}$ in $Z^{*}$. The forms are integrated over certain homology classes $\alpha^{*}=\alpha(A, x, \vartheta)^{*}$ and $t_{x} \partial \alpha^{*}$. The class $\alpha^{*}$ belongs to $H_{n-1}\left(Z^{*}-A^{*}, X^{*}\right)$ and is simply the class of $\alpha_{v}^{*}$ where $2 \alpha_{v}$ is the image of the map $\operatorname{Re} \dot{Z} \ni \xi \rightarrow \xi-i v(\xi), v \in V(A, X, \vartheta)$, and $\alpha_{v}^{*}$ is oriented by $x \xi \omega(\xi)>0$. Since $V(A, X, \vartheta)$ is one homotopy class, this defines $\alpha^{*}$ uniquely. Its boundary $\partial \alpha^{*} \in H_{n-2}\left(X^{*}-X^{*} \cap A^{*}\right)$ is an absolute class. The tube operation $t_{x}: H_{n-2}\left(X^{*}-X^{*} \cap A^{*}\right) \rightarrow H_{n-1}\left(Z^{*}-A^{*} \cup X^{*}\right)$ is generated by the boundary of a small 2-disk in the normal bundle of $X^{*}$ when its center moves on $X^{*}$. Because of the orientation $x \xi \omega(\xi)>0$, the homology class $\alpha^{*}$ depends in a very essential way on the parity of $n$. When $a \in \operatorname{Hyp}^{0}(9)$, the formulas (4), (5) are essentially due to Herglotz and Petrovsky. The formulas of Petrovsky are obtained by taking one residue onto $A^{*}$ in (4) and two successive residues onto $A^{*} \cap X^{*}$ in (5). These operations are well-defined only if $A^{*}$ and $A^{*} \cap X^{*}$ are nonsingular. The class $\alpha^{*}$ was introduced by Leray (1962).

A close study of the fundamental solutions of hyperbolic operators should involve behaviour near the wave fronts and also determination of supports and singular supports. The concept of a lacuna is basic in such a study at least for operators with constant coefficients. Before going into the details we shall consider wave propagation in general. As before, let $u(x)$ be a distribution defined in some open part $O$ of $R^{n}$ and think of it as describing the movement of an elastic $(n-1)$-dimensional medium. Let $C(u)$ be the maximal open part of $O$ where $u$ is a $C^{\infty}$-function. The complement of $C(u)$ is then the singular support of $u$, in our case the wave fronts. Let $L$ be a component of $C(u)$ and let $x$ be a point on the boundary $\partial L$ of $L$. We say that $u$ is sharp from $L$ at $x$ if $u$ has a $C^{\infty}$-extension from $L$ to $L \cap M$ where $M$ is some open neighbourhood of $x$. The physical implication of this is clear. An observer that moves at will in space-time and studies the movement $u$ in $L$ will not notice the part of the wave front at $x$ before actually crossing it. Measurements that he can make in $L$ will not indicate the singular behaviour at $x$. In the contrary situation, of a non-sharp wave front, measurements in $L$ close to $x$ indicate the presence of a front at $x$. Sharpness at all boundary points characterizes lacunas. More precisely, a component $L$ of $C(u)$ is said to be a lacuna of $u$ if $u$ has a $C^{\infty}$-extension from $L$ to $L$. When $u$ vanishes in $L, L$ is said to be a strong lacuna. Petrovsky only considers strong lacunas. The definition of lacuna given here follows a suggestion by L. Hörmander.

The lacunas for a hyperbolic operator $P$ are simply the lacunas of the distributions 
$x \rightarrow E(P, x, y)$, or, if $P$ has constant coefficients, the lacunas of the distribution $E(P)=$ $E(P, x)$. Consider the scalar case $P \in$ hyp $(\vartheta, m)$ and let $a \in$ Hyp $(\vartheta, m)$ be the principal part of $P$. We know that $E$ is holomorphic outside the wave front surface $W=W(A, \vartheta)$. Hence, if the singular support SSE of $E$ fills all of $W$, all lacunas are components of the comple. ment of $W$. This is the situation when $\operatorname{Re} \dot{A}=A-\{0\}$ in regular, when $n \leqslant 3$ and probably also when $n \leqslant 4$. However, if $n>4$, there are homogeneous operators $a$ in Hyp ( $\vartheta$ ) such that $W$ is not all of $\operatorname{SS} E(a)$. But we shall show that in all cases $W=\operatorname{SS} E\left(a^{k}\right)$ when $k$ is a large enough integer. At present, we cannot give a complete description of SS $E$ and $\mathrm{S} E$ for all $P$. In any case it is convenient to extend the lacuna definition by allowing as lacunas for $P$ components $L$ of the complement of $W$ having the property that $E(P)$ has a $C^{\infty}$-extension from $L$ to $L$. Such lacunas are said to be regular. In particular, the complement of the propagation cone $K=K(P)$ is a regular strong lacuna, called the trivial lacuna. With this definition, if $x$ belongs to a regular lacuna $L$ for $P$, then $E(a)$ is a polynomial of homogeneity $m-n$ in $L$ so that $L$ is a (regular) lacuna for $a$. In fact, it is immediate from (1) that $t^{n-m}(\partial / \partial x)^{\nu} E(P, \vartheta, t x) \rightarrow(\partial / \partial x)^{\nu} E(\alpha, \vartheta, x)$ in the distribution sense as $t \rightarrow 0$. Keeping $x$ in $L$, the derivatives $(\partial / \partial t x)^{\nu} E(P, \vartheta, t x)$ are supposed to have limits as $t \rightarrow 0$ and hence $(\partial / \partial x)^{\nu} E(a, \vartheta, x)=0$ in $L$ when $|v|>m-n$. By (1), $E(a, \vartheta, x)$ has homogeneity $m-n$ and this finishes the argument. A similar reasoning gives the same result when $P=P(x, \partial / \partial x)$ is hyperbolic with variable coefficients but as we shall stay with constant coefficients, we do not give the details. In any case we have seen that a component of $\operatorname{Re} Z-W(a, \vartheta)$ is a lacuna for $a \in \operatorname{Hyp}(\vartheta)$ if and only if $E(a, \vartheta, \cdot)$ is a polynomial there. Note that if $m<n$, then $E(a, \vartheta, \cdot)$ has to vanish $L$ so that $L$ is a strong lacuna. If $L$ is a regular lacuna not only for $a$ but for all powers of $a$ and $P \in$ hyp $(\vartheta)$ has principal part $a$, then (2) can be used to show that $E(P, \vartheta, \cdot)$ is an entire function in $L$ so that $L$ is a lacuna for $P$.

The Herglotz-Petrovsky-Leray formulas (4), (5) immediately give sufficient conditions for an $a \in \operatorname{Hyp}(\vartheta, m)$ to have non-trivial regular lacunas. In fact, by $(5)$, if

$$
\partial \alpha(A, x, \vartheta)^{*}=0 \quad \text { in } H_{n-2}\left(X^{*}-X^{*} \cap A^{*}\right)
$$

then $x$ belongs to a regular lacuna $L$ for all powers $a^{k}$ of $a$. If $m k-n<0, L$ is also a strong lacuna. The condition (6) is essentially due to Petrovsky. For this reason, regular lacunas $L$ such that (6) holds for all $x$ in $L$ are said to be Petrovsky lacunas. The corresponding condition connected with (4) and $m \geqslant n$, namely $\alpha^{*}=0$ in $H_{n-1}\left(Z^{*}-A^{*}, X^{*}\right)$ would imply that $x$ belongs to a regular strong lacuna for $a$. This case is, however, illusory. In fact, we shall prove in Part II that if $x$ is outside $W$ but in the propagation cone, then $\alpha^{*}$ has non-zero intersection number with $\Gamma(A, \vartheta)^{*} \in H_{n-1}\left(Z^{*}-X^{*}, A^{*}\right)$ and hence cannot vanish.

All the classical lacunas are Petrovsky lacunas. If, e.g. $n=2$, then $a$ is a product of 
linear factors and $\partial \alpha^{*}=0$ when $x \bar{\epsilon} \pm W$ so that the entire complement of $W$ consists of Petrovsky lacunas. Only the trivial lacuna is strong. Here it is of course quite elementary that $E(a, \vartheta, \cdot)$ is a polynomial in each component of $\operatorname{Re} Z-W$. If $n>2$ and $a=\Delta$ is the wave operator and $\vartheta=(1,0, \ldots, 0)$ then the propagation cone $K=K(A, \vartheta)$ is the forward light-cone $x_{1} \geqslant 0, x_{1}^{2}-x_{2}^{2}-\ldots-x_{n}^{2} \geqslant 0$ and $W=W(A, \vartheta)$ is its boundary. Here, if $x \in \stackrel{\circ}{K}, \partial \alpha^{*}$ vanishes or not according as $n$ is even or odd. This reflects the well-known fact that inside $K, E\left(\Delta^{k}, \vartheta, x\right)=c_{n, k}\left(x_{1}^{2}-x_{2}^{2}-\ldots-x_{n}^{2}\right)^{(2 k-n) / 2}$ where $c_{n, k}=0$ if $n$ is even and $2 k<n$ and $c_{n, k} \neq 0$ otherwise. Hence $\stackrel{\circ}{K}$ is a (regular) lacuna or not according as $n$ is even or odd. $\left({ }^{1}\right)$ This fact, sometimes called Huygens' principle, was the starting point of the theory of lacunas. There are examples of Petrorsky lacunas for every $m$ and $n$. These lacunas are also stable in the sense that they are not destroyed by small perturbations of $a$ within the class Hyp $(\vartheta, m)$. In fact, the Petrovsky condition (6) is stable under such perturbations. When $A^{*}$ is regular, the proof is immediate. In the general case we have to use the continuity properties of the local cones $\Gamma\left(A_{\xi}, \vartheta\right)$. The continuity properties of the wave front surface $W=W(A, \vartheta)$ show that no lacuna $L$ containing a piece of $W$ can be stable under all hyperbolic perturbations. In fact, there are operators $b \in \operatorname{Hyp}^{0}(\vartheta, m)$ such that $W(B, \vartheta)$ and hence also the singular support of $E(b, \vartheta, \cdot)$ comes arbitrarily close to any given ray in $W$.

It seems fruitful to try to prove or perhaps disprove some simple but strong statements about supports, singular supports and lacunas which are in agreement with known facts. Let $a \in \operatorname{Hyp}(\vartheta, m), E(a)=E(a, \vartheta, \cdot), K(A)=K(A, \vartheta)$ and let $a_{\xi} \in \operatorname{Hyp}(\vartheta)$ be the localizations. Our statements are

(i) all regular lacunas are Petrovsky lacunas

(ii) $\operatorname{SS} E(a)=\bigcup \mathrm{S} E\left(a_{\xi}\right)$ for $0 \neq \xi$ real

(iii) $E(a)$ is holomorphic outside $\operatorname{SS} E(a)$.

The statement

(iv) $m \geqslant n \Rightarrow \mathrm{S} E(a)=K(A)$

turns out not to be true for certain operators $a$ of the form $b\left(\xi^{\prime}\right) c\left(\xi^{\prime \prime}\right)$ where $\xi^{\prime}, \xi^{\prime \prime}$ is a partition of the coordinates $\xi$, but it would be enlightening to know precisely when it holds.

Petrovsky proved a weak form of (i) that can be paraphrased as follows: all stable lacunas for a strongly hyperbolic $a$ with non-singular $A^{*}$ are Petrovsky lacunas. Doing this, he used the topological machinery available at the time, in particular the results of Severi and Lefschetz on the topology of algebraic manifolds. At first, requiring stability under all perturbations of $a$ and using the fact that $E(a, \vartheta, x)$ is a holomorphic function also of $a$ when $x$ is outside the wave front surface, he can work in a generic situation where all

(1) The explicit formulas of Hadamard (1932) and M. Riesz (1948) show that this statement also holds for wave operators with variable coefficients. 
deformations of $A$ are permitted. One of his general results is the following one. Let $h(\xi)$ be a homogeneous non-zero polynomial of degree $m-n \geqslant 0$ and let $\varphi=\varphi_{h}$ be the corresponding holomorphic $(n-2)$-form on $A^{*}$. Assume that for some $\beta \in H_{n-2}\left(A^{*}\right)$ the integral $\int_{\beta} \varphi$ vanishes considered as a function of $a$. Then $\beta=0$. To prove this, Petrovsky analyses $H_{n-2}\left(A^{*}\right)$ explicitly. Assuming $\beta \neq 0$ he is able to find an $a$ with the property that $\int_{\beta} \varphi \neq 0$. The details involve a substantial amount of intuitive topology.

Working with the cohomology of $Z^{*}-A^{*}$ and $Z^{*}-A^{*} \cup X^{*}$ instead of the homology of $A^{*}$ and $A^{*} \cap X^{*}$ as Petrovsky does, we shall come much closer to proving (i). This will be done in Part II of our paper, but some of its results will be presented here. Our main tool is a well-known theorem by Grothendieck (1966) generalizing an earlier result by Atiyah and Hodge (1955). This theorem shows in particular that the cohomology of the complement of an algebraic hypersurface in projective space is spanned by all rational forms with poles on that hypersurface. It has an immediate application to our case. In fact, the forms that appear in (4), (5) applied to all powers of $a$ constitute a basis for all rational $(n-1)$ forms on $Z^{*}$ with poles on $A^{*}$ and $A^{*} \cup X^{*}$ respectively. Since the kernel of $t_{x}$ is zero, this shows that every regular lacuna for all powers of $a$ is a Petrovsky lacuna and that the only strong regular lacuna for all powers of $a$ is the trivial one. We shall also see that Grothendieck's theorem holds with a bound on the order of the poles depending only on $m$ and $n$, so that our statements are true for all sufficiently high powers of $a$ and hence (i) holds in a weak form regardless of the singularities of $A^{*}$. In this weak form and in combination with (3) it implies that the wave front surface $W(A, \vartheta)$ is the singular support of $E\left(a^{k}, \vartheta, \cdot\right)$ for large enough $k$. All statements (i) to (iii) hold when $n \leqslant 3$ or if $A^{*}$ is only mildly singular. The statement (iv) is probably true when $n \leqslant 3$ and it holds in the following weak form: when $m \geqslant n, a$ has no stable strong lacunas inside $K$.

In physics, hyperbolic systems are more important than the scalar operators which have occupied us here. However, every component of the fundamental solution $E(P, \vartheta, \cdot)$ where $P \in \operatorname{Hyp}_{N}(\vartheta)$ is a sum of derivatives of $E(\operatorname{det} P, \vartheta, \cdot)$. Hence it suffices to study supports, singular supports and lacunas for scalar distributions of this form. For instance, it seems desirable to show that if $a \in \mathrm{Hyp}(\vartheta)$ and $Q$ is relatively prime to $a$, then every regular lacuna for $Q(D) E(a, \vartheta, \cdot)$ is a Petrovsky lacuna for $a$. This situation leads to problems about the cohomology of rational forms that will be treated in part II.

This introduction combined with the table of contents should give a fair idea of the problems we pose and our main results. A preliminary outline has been published earlier (Gårding 1969). Finally, we should like to thank Lars Hörmander for his decisive contributions to our paper. We are also grateful to St Catherine's College, Oxford, under whose aegis this study was initiated. 


\section{Contents}

Introduction ..............................109

Chapter 1. Distributions and the Fourier-Laplace transform

1. Generalities about distributions . . . . . . . . . . . . . . . . . 118

2. Inverse Fourier-Laplace transforms of functions holmorphic in tubes . . . . . 124

Chapter 2. Fundamental solutions of hyperbolic operators with constant coefficients

3. Hyperbolic differential operators and hyperbolic polynomials . . . . . . . . . 126

4. Fundamental solutions of scalar hyperbolic operators. Localization. Analytic continuation . . . . . . . . . . . . . . . . . . . 142

5. The geometry of hyperbolic surfaces. Semi-continuity of the local cones. The wave front surface . . . . . . . . . . . . . . . . . . . . . 148

6. Vector fields and cycles . . . . . . . . . . . . . . . . . . . . 160

7. Fundamental solutions expressed as rational integrals . . . . . . . . . . 169 Chapter 3. Sharp fronts and lacunas

8. Supports and singular supports of fundamental solutions . . . . . . . . . . . . 179

9. Sharp fronts, lacunas, regular lacunas . . . . . . . . . . . . . . . . 182

10. Stability, Petrovsky lacunas . . . . . . . . . . . . . . . . . 184

11. Remarks about systems . . . . . . . . . . . . . . . . . . 188

References ......................... . . 188

\section{Chapter 1. Distributions and the Fourier-Laplace transform}

This chapter will present some general material to be used later on.

\section{Generalities about distributions}

Distributions on a manifold. Let $X$ be a paracompact $C^{\infty}$-manifold of dimension $n$. Let $x$ be a point on $X,\left(x_{1}, \ldots, x_{n}\right)$ the coordinates of $x$ and put

$$
(\partial / \partial x)^{\nu}=\left(\partial / \partial x_{1}\right)^{\nu_{1}} \ldots\left(\partial / \partial x_{n}\right)^{v_{n}}
$$

where $v_{1}, \ldots$ are integers $\geqslant 0$. The order of this derivative is $|v|=v_{1}+\ldots+v_{n}$. Let $C(X)=$ $C^{\infty}(X)$ be the space of all complex $C^{\infty}$-functions on $X$ and $C_{0}(K)$ the subspace of $C(X)$ whose elements have supports in a given compact subset $K$ of $X$. Let $C_{0}(X)$ be the union of all $C_{0}(K)$. Topologize $C(X)$ by locally uniform convergence of all derivatives and topo- 
logize $C_{0}(X)$ as the inductive limit of all $C_{0}(K)$ with the topology induced by $C(X)$ as $K$ runs through a sequence of compacts tending to $X$. Consider also $n$-forms on $X$,

$$
\varphi(x)=g(x) d x, \quad d x=d x_{1} \wedge \ldots \wedge d x_{n},
$$

with densities $g \in C(X)$. Assume $X$ to be oriented by some $\varrho(x)>0$. Let $\Omega(X), \Omega_{0}(X), \Omega_{0}(K)$ be the spaces of forms $\varphi$ such that $\varphi / \varrho$ belongs to $C(X), C_{0}(X), C_{0}(K)$ respectively, topologized in the obvious way. If $X$ is an open subset of $R^{n}$, we always take $\varrho(x)=d x=$ $d x_{1} \wedge \ldots \wedge d x_{n}$. The distributions on $X$ are by definition the elements of the dual $C^{\prime}(X)$ of $\Omega_{0}(X)$. The support of a distribution $f$ will be denoted by $\mathrm{S}(f)$. We let $C_{0}^{\prime}(X)$ be the space of distributions with compact supports. Every locally integrable function $f(x)$ gives rise to a distribution

$$
\varphi \rightarrow \int_{X} f(x) \varphi(x), \quad \varphi \in \Omega_{0}(X)
$$

We identify $f$ and this distribution. Also in the general case, distributions are written as $f(x), g(x), \ldots$ and we employ one or other of the two notations

$$
(f, \varphi)=\int_{X} f(x) \varphi(x)
$$

for the value of $f$ at $\varphi$. If $X$ is an open subset of $R^{n}$, we identify $\varphi$ with $g(x) d x$ and write

$$
(f, g)=\int_{x} f(x) g(x) d x
$$

Note that $C_{0}(X)$ and hence also $C(X)$ is dense in $C^{\prime}(X)$. The derivatives $(\partial / \partial x)^{p} f(x)$ of a distribution are defined in the domain $V$ of the coordinates $x_{1}, \ldots, x_{n}$ by

$$
\int(\partial / \partial x)^{v} f(x) g(x) d x=(-1)^{|v|} \int f(x)(\partial / \partial x)^{v} g(x) d x
$$

where $g(x) d x \in \Omega_{0}(V)$. Locally, a distribution is a finite sum of derivatives of continuous functions. Let $\left(x_{1}, \ldots, x_{n}\right)$ and $\left(y_{1}, \ldots, y_{n}\right)$ be coordinates with a common domain. The chain rule of differentiation for functions,

$$
\partial f / \partial y_{i}=\sum_{k}\left(\partial x_{k} / \partial y_{i}\right) \partial f / \partial x_{k}
$$

extends by continuity to distributions.

The singular support of a distribution of $f$ is the complement of the maximal open subset $U$ of $X$ where $f$ equals a $C^{\infty}$-function. It will be denoted by $\operatorname{SS}(f)$. 
Temperate distributions. The Fourier transform. When $g \in C\left(R^{n}\right)$, put

$$
|g|_{N}=\sup (1+|x|)^{N}\left|\partial^{\prime} g(x)\right|, x \in R^{n},|v| \leqslant N
$$

where $|x|$ denotes some norm on $R^{n}$, and let $S=S\left(R^{n}\right)$ be all $g \in C\left(R^{n}\right)$ for which all $|g|_{N}$ are finite. Topologized by the norms $|g|_{N}, S$ is a Fréchet space, the space of temperate test-functions, and its dual $\boldsymbol{S}^{\prime}=\boldsymbol{S}^{\prime}\left(R^{n}\right)$ is the space of temperate distributions (Schwartz 1950).

Let $Z=\{\xi, \eta, \ldots\}$ and $Z^{\prime}=\{x, y, \ldots\}$ be two complex vector spaces of dimension $n$. We shall always choose biorthogonal coordinates $\left(\xi_{1}, \ldots\right)$ and $\left(x_{1}, \ldots\right)$ so that $x \xi=x_{1} \xi_{1}+\ldots+$ $x_{n} \xi_{n}$ and $|x|,|\xi|$ will denote unspecified norms. The Fourier transform

$$
\mathcal{I} f(\xi)=\int e^{-i x \xi} f(x) d x, \quad x, \xi \text { real }
$$

is a linear homeomorphism $S \rightarrow S$ with inverse

$$
\mathcal{F}^{-1} f(x)=(2 \pi)^{-n} \int e^{i x \xi} f(\xi) d \xi
$$

that extends to a linear homeomorphism $S^{\prime} \rightarrow S^{\prime}$, also denoted by $\mathcal{F}$.

When $g \in C_{0}\left(\operatorname{Re} Z^{\prime}\right)$, its Fourier transform extends to the Fourier-Laplace transform

$$
(\mathcal{F} g)(\xi+i \eta)=\int e^{-i x(\xi+i \eta)} g(x) d x, \quad \xi, \eta \text { real, }
$$

which is an entire holomorphic function. In connection with Fourier transforms it is often convenient to use the imaginary gradient

$$
D=i^{-1} \partial / \partial x
$$

which has the property that $\xi \mathfrak{F}=\mathfrak{I} D$.

Distributions in one variable. Let $X=R^{n}$ and let $f \in C^{\prime}(R)$. Then $f\left(x_{1}\right)$ can be considered as an element of $C^{\prime}(X)$ defined by

$$
(f, g)=\int\left(\int f\left(x_{1}\right) g\left(x_{1}, \ldots, x_{n}\right) d x_{1}\right) d x_{2} \wedge \ldots \wedge d x_{n}
$$

where all forms are positive and $g \in C_{0}(X)$. The injection $C^{\prime}(R) \rightarrow C^{\prime}(X)$ obtained in this way is continuous. When $X$ is a manifold, there is a similar construction. Let $s(x) \in C(X)$ be real and such that grad $s(x) \neq 0$ everywhere. The distribution $f(s(x)) \in C^{\prime}(X), f \in C^{\prime}(R)$, is defined by 


$$
\int f(s(x)) \varphi(x)=\int_{V} f(t) h(t) d t,
$$

where $\varphi \in \Omega_{0}(X), V \subset R$ is the (open) range of $s$ and

$$
h(t)=\int_{s(x)=t} \varphi_{t}(x), \quad \varphi(x)=\varphi_{t}(x) \wedge d t
$$

with $V$ and $s(x)=t$ oriented so that $d t>0$ and $\varrho_{t}(x)>0$ respectively, $\varrho(x)>0$ being the orientation of $X$. In particular, if $V \ni 0$,

$$
\int \delta^{(j)}(s(x)) \varphi(x)=(-1)^{j} h^{(j)}(0),
$$

where $\delta$ is the Dirac measure on $R$. We note that also

$$
h(t)=\int_{X} \delta(s(x)-t) \varphi(x)
$$

For reference we state the following lemma whose proof is left to the reader.

1.2. Lемма. The injection $C^{\prime}(R) \rightarrow C^{\prime}(X)$ defined by $(1.1)$ is continuous.

Distributions which are boundary values of holomorphic functions. Let $A$ be an open connected part of the upper half-plane $y_{1}>0$ of the complex $\left(x_{1}+i y_{1}\right)$-plane whose boundary contains an interval $I$ of the real axis and let $B \subset R^{n-1}$ be open. Let

$$
f(x+i y)=f\left(x_{1}+i y_{1}, x_{2}, \ldots, x_{n}\right)
$$

be continuous in $A \times B$ and holomorphic in its first variable and assume that

$$
f(x+i y)=O\left(y_{1}^{-N}\right), \quad y_{1} \downarrow 0
$$

for some integer $N>0$, locally uniformly when $x \in I \times B$. Then

$$
f(x)=\lim f(x+i y), \quad y_{1} \downarrow 0
$$

exists as a distribution, the limit being taken in the sense that

$$
\lim \int f(x+i y) g(x) d x, \quad y_{1} \downarrow 0,
$$

exists for every $g \in C_{0}(I \times B)$. Moreover, if the limit vanishes, so does $f$. In fact, let $f_{1}, f_{2}, \ldots$ be successive integrals of $f$ with respect to the first variable taken from a fixed point in $A$. Then, using (1.3) one has $f_{N}(x+i y)=O\left(\log y_{1}^{-1}\right)$ and still another integration shows that, 
as $y_{1} \rightarrow 0, f_{N+1}(x+i y)$ tends locally uniformly to a continuous function $f_{N+1}(x)$ on $I \times B$. Now, by integrations by parts,

$$
\int f(x+i y) g(x) d x=(-1)^{N+1} \int f_{N+1}(x+i y)\left(\partial / \partial x_{1}\right)^{N+1} g(x) d x
$$

and hence the limit (1.4) exists and defines a distribution $f(x)=\left(\partial / \partial x_{1}\right)^{N+1} f_{N+1}(x)$ on $I \times B$. If this distribution vanishes, $f_{N+1}(x)$ is a polynomial of degree $<N+1$ in $x_{1}$ when $x_{2}, \ldots$ are fixed so that, by elementary function theory, $f_{N+1}(x+i y)$ is the same polynomial with argument $x_{1}+i y_{1} \in A$. Hence $f$ vanishes in $A \times B$.

In case $A$ is a band $0<y_{1}<$ const, $B=R^{n-1}$ and (1.3) is replaced by

$$
f(x+i y)=O\left(y_{1}^{-N}(1+|x|)^{N}\right)
$$

then

$$
f_{N+1}(x+i y)=O\left((1+|x|)^{2 N+1}\right)
$$

so that

$$
f(x)=\left(\partial / \partial x_{1}\right)^{N+1} f_{N+1}(x)
$$

is a temperate distribution. Note that in this case, (1.5) holds when $g \in S$ and that the limit (1.4) exists also when $g(x)=g_{y}(x)$ depends on $y$ provided $g_{y} \rightarrow g$ in $S$ when $y_{1} \rightarrow 0$.

Later on, in section 7 , we shall employ certain explicit distributions on $R$ which are boundary values of functions analytic in the upper halfplane. We define them here and state their main properties.

Let $s, z$ be complex numbers and put

$$
\chi_{s}(z)=\Gamma(-s) e^{-\pi i s} z^{s}, \quad s \neq 0,1,2, \ldots, \quad 0<\arg z<\pi
$$

Note that

$$
\operatorname{Re} s<0 \Rightarrow \chi_{s}(z)=i^{-s} \int_{0}^{\infty} \varrho^{-s-1} e^{i \ell z} d \varrho, \quad \arg i=\pi / 2
$$

Hence, when Re $s<0, \chi_{s}$ is a constant times the Fourier-Laplace transform of a function that vanishes on the negative axis and equals $\varrho^{-s-1}$ on the positive axis. By the properties of the gamma function,

$$
\chi_{s}^{\prime}(z)=\chi_{s-1}(z)
$$

Considered as a function of $s, \chi_{s}(z)$ has simple poles at $s=q=0,1,2, \ldots$. We shall need the first and second coefficients of the Laurent expansion of $\chi_{s}$, viz

$$
\chi_{s+t}(z)=-t^{-1} \chi_{s}^{0}(z)+\chi_{s}(z)+O(t)
$$


where the first term vanishes unless $s=q=0,1,2, \ldots$ Since (1.5) does not define $\chi_{s}$ when $s=q$, we are free to denote the constant term by $\chi_{a}$ in that case and it turns out to be convenient to do so. Both coefficients can be computed explicitly. By (1.6) and the properties of the gamma function,

$$
\chi_{a+t}(z)=-\prod_{0}^{a}(q-k+t)^{-1} \Gamma(1-t) e^{-\pi i t} z^{a+t} .
$$

Hence

$$
\begin{aligned}
& \chi_{q}^{0}(z)=z^{q} / q ! ; \quad q=0,1,2, \ldots, \\
& \chi_{s}^{0}(s)=0 \text { when } s \neq 0,1,2, \ldots,
\end{aligned}
$$

and, multiplying (1.9) by $t$ and taking the derivative at $t=0$, we get

$$
\chi_{q}(z)=\left.\frac{d}{d t} t \chi_{q+t}(z)\right|_{t=0}=(q !)^{-1} z^{Q}\left(\log z^{-1}+c_{q}+\pi i\right)
$$

where

$$
c_{q}=\Gamma^{\prime}(1)+\sum_{1}^{q} k^{-1}, c_{0}=\Gamma^{\prime}(1)
$$

It follows from (1.12) that (1.8) holds also when $s=0,1, \ldots$ and hence for all $s$. We note in passing that (1.9) implies that

$$
\left.\frac{d}{d t} t \chi_{s+t}(z) f(t)\right|_{t=0}=\chi_{s}(z) f(0)-\chi_{s}^{0}(z) f^{\prime}(0)
$$

when $f$ is holomorphic at the origin. In fact, in view of (1.9), both sides equal the constant term in the Laurent expansion of $t_{\chi_{s+t}}(z) f(t)$ at $t=0$.

In view of our earlier statements about boundary values of analytic functions, the distributions

$$
\chi_{s}(x)=\lim \chi_{s}(x+i y), \quad y \downarrow 0, \quad x \in R
$$

exist for all $s$. As functions of $s$, they have the same properties as before, in particular the property (1.8). Passing to the limit in (1.12) we get

$$
q ! \chi_{q}(x)=x^{q}\left(\log |x|^{-1}+c_{q}\right)+2^{-1} \pi i(1+\operatorname{sgn} x) x^{q} .
$$

Hence, if $\sigma_{q} \in C^{\prime}(R)$ is defined by

$$
2 \pi i \sigma_{q}(x)=\chi_{q}(x)-(-1)^{q} \chi_{q}(-x), \quad q=0, \pm 1, \ldots,
$$

the logarithm gets eliminated and we have 


$$
\begin{aligned}
& \sigma_{q}(x)=2^{-1}(\operatorname{sgn} x) x^{q} / q !=2^{-1}(\operatorname{sgn} x) \chi_{q}^{0}(x), \quad q=0,1, \ldots \\
& \sigma_{q}(x)=\delta^{(-q-1)}(x), \quad q=-1,-2, \ldots .
\end{aligned}
$$

In fact, the first formula (1.17) follows from (1.15) and (1.16) and the second from the first if we observe that (1.8) and (1.16) imply that $\sigma_{q}^{\prime}=\sigma_{q-1}$.

\section{Inverse Fourier-Laplace transforms of functions holomorphic in tubes}

Duality of cones. Let $\Gamma \subset \operatorname{Re} Z$ be a cone with its vertex at the origin, i.e. such that $\lambda \Gamma=\Gamma$ when $\lambda>0$. Define the dual cone $\Gamma^{\prime} \subset \operatorname{Re} Z^{\prime}$ by

$$
\Gamma^{\prime}=\{x ; x \xi \geqslant 0, \forall \xi \in \Gamma\} \text {. }
$$

Then $\Gamma^{\prime}=\overline{\Gamma^{\prime}}$ is a closed convex cone. In fact $\Gamma^{\prime}=H\left(\Gamma^{\prime}\right)$ where $H$ denotes the closed convex hull. Let

$$
\Gamma^{\prime \prime}=\left\{\xi ; \xi x \geqslant 0, x \in \Gamma^{\prime}\right\} \subset \operatorname{Re} Z
$$

be the dual of $\Gamma^{\prime}$. Since $H(\Gamma)$ is the intersection of all closed halfspaces containing $\Gamma$, we have $\Gamma^{\prime \prime}=H(\Gamma)$. Now, obviously,

$$
\left(\Gamma_{2}^{\prime}+\Gamma_{2}^{\prime}\right)^{\prime}=\Gamma_{1}^{\prime \prime} \cap \Gamma_{2}^{\prime \prime}
$$

so that, taking the dual of both sides,

$$
\left(\Gamma_{1} \cap \Gamma_{2}\right)^{\prime}=H\left(\Gamma_{1}^{\prime}+\Gamma_{2}^{\prime}\right) \text {. }
$$

It is clear that $\Gamma$ is contained in a hyperplane $y \xi=0, y$ fixed in $\operatorname{Re} Z^{\prime}$, if and only if $\Gamma^{\prime} \ni x$ implies $x+t y \in \Gamma^{\prime}$ for all real $t$. Hence if $\Gamma$ is convex, $\Gamma$ is open if and only if $\Gamma^{\prime}$ is proper in the sense that $\Gamma^{\prime}$ does not contain any straight lines. Let $\Gamma$ be open and convex so that $\bar{\Gamma}=H(\Gamma)$. Its dual $K=\Gamma^{\prime}$ is then closed, convex and proper. Clearly, $\xi \in \Gamma$ if and only if $\xi x>0$ when $x \in \overleftarrow{K}=K-0$. All $\eta$ such that $\Gamma+t \xi \subset \Gamma$ for all real $t$ constitute a linear space $E$, the edge of $\Gamma$. Obviously, $K$ is contained in its orthogonal complement $E^{0}=\{y ; y E=0\}$. It also spans this complement. In fact, if $\eta K=0$ and $\xi \in \Gamma$, then $(\xi+t \eta) \dot{K}=\xi \dot{K}>0$ for all $t$ so that $\eta \in E$. If $E=0, \Gamma$ is proper and $K$ has a non-empty interior $\stackrel{\circ}{K}$. One verifies that $x \in \dot{K}$ if and only if, for every real $c$, the half-spaces $x \xi \leqslant c$ have compact intersections with $\bar{\Gamma}$.

Inverse Fourier-Laplace transforms. When $g \in C_{0}\left(\operatorname{Re} Z^{\prime}\right)$, its Fourier-Laplace transform

$$
\mathcal{F}(\xi+i \eta)=\int e^{-i x(\xi+i \eta)} g(x) d x
$$

is an entire function and obvious estimates of 


$$
(\xi+i \eta)^{\nu} \mathcal{F} g(\xi+i \eta)=\int e^{-i x(\xi+i \eta)} D^{\nu} g(x) d x
$$

show that to every $M>0$ there is a $c(M)$ such that

$$
|\mathfrak{I} g(\xi+i \eta)| \leqslant c(M)(1+|\xi+i \eta|)^{-M} e^{h(\eta)}
$$

where

$$
h(\eta)=\max x \eta, \quad \eta \in \mathbb{S}(g)
$$

is the so-called support function of $\mathbf{S}(g)$.

The estimate (2.3) leads naturally to a definition of an inverse Fourier-Laplace transform by duality. Suppose that $F(\xi+i \eta)$ is holomorphic in $\operatorname{Re} Z+i \Delta$ where $\Delta \subset \operatorname{Re} Z$ is open and connected and suppose that

$$
|F(\xi+i \eta)| \leqslant c(\eta)(1+|\xi+i \eta|)^{N}, \quad \eta \in \Delta,
$$

for some $N$ and some $c(\eta)$ locally bounded on $\Delta$. Then

$$
f(x)=(2 \pi)^{-n} \int F(\xi+i \eta) e^{i x(\xi+i \eta)} d \xi, \quad \eta \in \Delta,
$$

defines a distribution $f$, called the inverse Fourier-Laplace transform of $F^{\prime}$ and denoted by $\mathcal{I}^{-1} f$. Here $(2.5)$ has to be interpreted as

$$
(f, \check{g})=(2 \pi)^{-n} \int F(\xi+i \eta) \mathcal{F}(\xi+i \eta) d \xi, \quad \eta \in \Delta,
$$

where $\breve{g}(x)=g(-x)$ and $g \in C_{0}\left(\operatorname{Re} Z^{\prime}\right)$. The estimates (2.3), (2.4) show that the integral is absolutely convergent; by Cauchy's theorem, it is independent of $\eta$.

The construction of fundamental solutions of hyperbolic operators relies on the following well-known variant of the Paley-Wiener theorem.

2.5. THЕ ОR EM. Let F satisfy (2.4) and suppose in addition that $\Delta$ is convex and

$$
t \Delta \subset \Delta, \quad c(\eta)=c(\eta)
$$

when $t \geqslant 1$ and let $\Gamma=\bigcup s \Delta$ for $s>0$. Then the support of $f=\mathcal{F}^{-1} F$ is contained in the cone $K$ dual to $-\Gamma$. If $\Delta=\Gamma$ and (2.4) is replaced by

$$
|F(\xi+i \eta)| \leqslant c(\eta)(1+|\xi+i \eta|)^{N}\left(1+|\eta|^{-N}\right),
$$

where now $c(t \eta)=c(\eta)$ for all $t>0$, then 


$$
F_{-}(\xi)=\lim F(\xi+i t \eta), \quad t \downarrow 0, \quad \eta \in-\Gamma
$$

is a temperate distribution and

$$
f=\mathfrak{F}^{-1} \boldsymbol{F}_{-}
$$

Note. It is easy to see that $\left(2.4^{\prime}\right)$ completely characterizes Fourier-Laplace transforms of temperate distributions with support in $K=-\Gamma^{\prime}$.

Proof. We know the right side of $\left(2.5^{\prime}\right)$ to be independent of $\eta$ and we can majorize the integrand using (2.3) and (2.4). If $\mathrm{S}(\breve{g}) \cap K$ is empty and $\mathrm{S}(\breve{g})$ is convex, there is an $\eta \in \Gamma$ such that $x \eta \geqslant 0$ on $\mathrm{S}(\check{g})$, i.e. $x \eta \leqslant 0$ on $\mathbf{S}(g)=-\mathrm{S}(\check{g})$ and then (2.3) holds with no exponential factor on the right. Moreover, $t \eta \in \Delta$ when $t$ is large enough. Hence, replacing $\eta$ by $t \eta$ in $\left(2.5^{\prime}\right)$ and letting $t \rightarrow \infty$, the integral vanishes. Hence $\mathrm{S}(f) \subset K=-\Gamma^{\prime}$. When $\left(2.4^{\prime}\right)$ holds, then, fixing $\eta \in \Gamma$, replacing $\eta$ by $t \eta$ and letting $t \downarrow 0$, our earlier remarks on distributions which are boundary values of analytic functions show that the limit (2.8) exists, that it is a temperate distribution and that $(f, \breve{g})=(F, \mathfrak{I} g)$. This proves $(2.9)$.

\section{Chapter 2. Fundamental solutions of hyperbolic operators with constant coefficients}

Section 3 defines hyperbolic operators and states their main properties. In section 4 , we shall get fundamental solutions expressed as inverse Fourier-Laplace transforms. This section ends with an outline of how to get the Herglotz-Petrovsky-Leray formulas. This will be carried out in detail in section 7 , which relies on a close analysis of the geometry of hyperbolic surfaces including the semi-continuity of the local cones and a study of the wave front surface (section 5). The vector fields and cycles used in section 7 are presented in section 6 .

\section{Hyperbolic differential operators and hyperbolic polynomials}

Let $X$ be an open part of $R^{n}$ and

$$
P(x, D)=\sum P_{v}(x) D^{\nu}, \quad D=D_{x}=i^{-1} \partial / \partial x,
$$

a differential operator on $X$ with smooth complex coefficients. A (right) fundamental solution of $P$ is, by definition, a distribution $E(P, x, y)$ on $X \times X$ such that

$$
P\left(x, D_{x}\right) E(P, x, y)=\delta(x-y) .
$$

$P$ is said to be hyperbolic in $X$ if $E$ can be chosen so that it vanishes when $x$ is close to $y$ 
except when $x$ belongs to some proper cone with its vertex at $y$. The same definition applies to differential operators whose coefficients are square matrices of some fixed order.

We shall only deal with the case when $P$ has constant coefficients. We say that $P$ is hyperbolic with respect to $\vartheta=\left(\vartheta_{1}, \ldots, \vartheta_{n}\right) \in \operatorname{Re} Z$ if $P$ has a fundamental solution $E=$ $E(P, \vartheta, x)$ now satisfying $P E=\delta(x)$ and having support in some closed cone $K$ with its vertex at the origin such that $x \vartheta>0$ on $\dot{K}=K-\{0\}$. Here the coefficients of $P$ are supposed to be $r \times r$ matrices and $I$ is the unit $r \times r$ matrix. By a translation, this is equivalent to hyperbolicity in the sense above.

3.1. Lемм А. When $P$ is hyperbolic with respect to $\vartheta$, the fundamental solution $E(P, \vartheta, x)$ is unique.

Proof. It is well known that the space of distributions with support in a proper cone $K$ with its vertex at the origin forms a convolution algebra and that the space of distributions with support in a closed half-space whose interior contains $\dot{K}$, forms a module under convolution with this algebra. (Schwartz 1950, II, p. 32.) The same holds for distributions whose values are square matrices. Put $\tilde{P}(x)=P(D) \delta(x) I$ so that $P(\partial / \partial x) f(x)=(\tilde{P} * f)(x)$. Then $\tilde{P} * E=\delta I$ is the unit in the convolution algebra of such distributions with support in $K$. Since the corresponding scalar convolution algebra is commutative, this shows that also $E * \widetilde{P}=\delta I$. Now let $F$ be another fundamental solution of $P$ with support in $x \vartheta \geqslant 0$. Then $F=\delta * F=E * \widetilde{P} * F=E$. This finishes the proof.

The convolution algebra also gives a proof of the following lemma that reduces the study of hyperbolic operators with constant coefficients to the scalar case.

3.2. Limma. A differential operator $P(D)$ whose coefficients are square matrices is hyperbolic with respect to $\vartheta$ if and only if its determinant $(\operatorname{det} P)(D)$ has that property.

Proof. Suppose first that $P$ is hyperbolic with respect to $\vartheta$ and let $E$ be the corresponding fundamental solution. Then $\tilde{P} * E=\delta I$ and, taking determinants in the sense of convolution, this gives $\operatorname{det} \widetilde{P} * \operatorname{det} E=\delta$. Since $\mathrm{S}(\operatorname{det} E) \subset K$ and $\operatorname{det} \widetilde{P}=(\operatorname{det} P)^{\sim}$, this shows that $\operatorname{det} P$ is hyperbolic with respect to $\vartheta$. On the other hand, suppose this to be true and let $E(\operatorname{det} P, \vartheta, x)$ be the corresponding fundamental solution. Let $Q(D)$ be the matrix of cofactors in $P(D)$ so that $P Q=(\operatorname{det} P) I$. Then

$$
E(P, \vartheta, x)=Q(D) E(\operatorname{det} P, \vartheta, x) I .
$$

In fact, $\mathrm{S}(E) \subset K$ and

$$
P(D) E(P, \vartheta, x)=(\operatorname{det} P)(D) E(\operatorname{det} P, \vartheta, x) I=\delta(x) I .
$$


For scalar operators $P(D)$, hyperbolicity is equivalent to an algebraic property of its characteristic polynomial

$$
P(\xi)=\sum P_{\nu} \xi^{\nu}
$$

of $P$. Assume $P$ to be of degree $m$ and let

$$
P_{m}(\xi)=\sum P_{\nu} \xi^{\nu}, \quad|\nu|=m,
$$

be the characteristic polynomial of the principal part $P_{m}(\partial / \partial x)$ of $P(\partial / \partial x)$.

3.4. Definition. The polynomial $P(\xi)$ is said to be hyperbolic with respect to $\vartheta \in \operatorname{Re} Z$ if $P_{m}(\vartheta) \neq 0$ and $P(\xi+t \vartheta) \neq 0$ when $\xi$ is real and $\operatorname{Im} t$ is less than some fixed number. The space of such polynomials will be denoted by hyp $(\vartheta, m)$. When $m$ is not specified we write hyp $(\vartheta)$. A polynomial $P(\xi)$ whose coefficients are $r \times r$ matrices is said to be hyperbolic with respect to $\vartheta$ if $\operatorname{det} P(\xi)$ has that property. The space of such polynomials will be denoted by hyp $\operatorname{hy}_{r}(\vartheta)$.

We now have

3.5. ThEOREM. A differential operator $P(D)$ whose coefficients are $r \times r$ matrices is hyperbolic with respect to $\vartheta$ if and only if its characteristic polynomial belongs to $\operatorname{hyp}_{r}(\vartheta)$.

In view of Lemma 3.2, it suffices to consider the scalar case $r=1$. The theorem is due to Gårding (1950). It results from Theorem 5.6.1 and 5.6.2 in Hörmander's book Linear Partial Differential Operators (1963), hereafter referred to as $H$. The book also gives short proofs of the most important properties of hyperbolic polynomials. For the reader's convenience, we shall repeat some of them.

We are going to sketch a proof of the necessity part of Theorem 3.5. The sufficiency will follow from the construction of fundamental solutions in the next section. We have to show that if the operator $P(D)$ is hyperbolic with respect to $\vartheta$ then $P \in$ hyp $(\vartheta)$. In fact, let $E(x)$ be a fundamental solution of $P$ with support in a proper cone $K$ such that $x \vartheta>0$ on $\dot{K}$. Let $\varphi \in C_{0}^{\infty}\left(\operatorname{Re} Z^{\prime}\right)$ be 1 in a neighborhood of the origin. Then

$$
P(D) f(x)=\delta(x)+g(x)
$$

where $f=\varphi E$ and $g=P(D)(\varphi-1) E$ are distributions with compact supports and

$$
h(\eta)=\max x \eta, \quad x \in \mathbf{S}(g)
$$

is positive homogeneous in $\eta, h(\lambda \eta)=\lambda h(\eta)$ when $\lambda \geqslant 0$, and, since $\mathbf{S}(g)$ is a compact part of $\dot{K}, h(-\vartheta)<0$. Taking Fourier-Laplace transforms,

$$
P(\xi) F(\xi)=1+G(\xi), \quad \xi \in Z,
$$

where $F=\mathcal{F}, G=\mathcal{F} g$ are entire functions and 
for some $C, N>0$. Hence

$$
|G(\xi)| \leqslant C(1+|\xi|)^{N} e^{h(\operatorname{Im} \xi)}
$$

$$
P(\xi)=0 \Rightarrow h(\operatorname{Im} \xi) \geqslant-c \log (2+|\xi|),
$$

where $c>0$ is another constant. In particular, $P \neq 0$. Let $P_{m}$ be the principal part of $P$. If $P_{m}(\vartheta)=0$, it is easy to see that the equation $P(\xi+t \vartheta)=0$ has a root $t=t(\xi)$ such that $\operatorname{Im} t \rightarrow-\infty$ as $\xi$ tends to some $\xi^{0}$ along a suitable path. But then $h(\operatorname{Im}(\xi+t \vartheta))=h(\operatorname{Im} t \vartheta)+$ $O(1)$ tends to $-\infty$ and this contradicts (3.6) with $\xi$ replaced by $\xi+t \vartheta$. Hence $P_{m}(\vartheta) \neq 0$. Further, (3.6) implies

$$
P(\xi+t \vartheta)=0 \Rightarrow h(\operatorname{Im} t \vartheta) \geqslant-c_{1} \log (2+|\xi|)
$$

where now $\xi$ is real and $c_{1}$ is a new constant. A well-known lemma by Seidenberg-Tarski (H Appendix, Lemma 2.1) shows that the logarithm in the right can be replaced by a constant and this finishes the proof.

We shall mainly be concerned with homogeneous hyperbolic polynomials $a(\xi)$. In view of the definition above and the homogeneity of $a$, we have $a \in$ hyp $(\vartheta, m)$ if and only if

$$
\xi \text { real, } \operatorname{Im} t \neq 0 \Rightarrow a(\xi+t \vartheta) \neq 0
$$

or, equivalently,

$$
\xi \text { real } \Rightarrow a(\xi+t \vartheta)=0 \text { has } m \text { real roots } t
$$

3.8. Definition. The space of homogeneous polynomials $a \in$ hyp $(\vartheta, m)$ will be denoted by Hyp $(\vartheta, m)$. An $a \in \operatorname{Hyp}(\vartheta, m)$ is said to be strongly hyperbolic if the zeros of $a(\xi+t \vartheta)$ are all different when $\xi$ is not proportional to $\vartheta$. The space of these polynomials will be denoted by $\mathrm{Hyp}^{0}(\vartheta, m)$.

When $m$ is not specified, we write Hyp $(\vartheta)$ and $\mathrm{Hyp}^{0}(\vartheta)$. Note that $\mathrm{Hyp}(\vartheta)=$ Hyp $(-\vartheta)$.

The interest of strongly (or strictly) hyperbolic polynomials is that they remain hyperbolic under addition of arbitrary lower order terms, i.e.

$$
a \in \operatorname{Hyp}^{0}(\vartheta, m) \text {, degree } b<m \Rightarrow a+b \in \operatorname{ehyp}(\vartheta, m) .
$$

This will be proved later. We shall sometimes let $\operatorname{hyp}^{0}(\vartheta, m)$ denote the space of polynomials in hyp $(\vartheta, m)$ whose principal parts are strongly hyperbolic. This class also gives rise to hyperbolic operators with variable coefficients. In fact, if $X \subset R^{n}$ is open and $P(x, \partial / \partial x)$ is a differential operator with smooth coefficients and $P(x, \xi) \in \operatorname{hyp}^{0}(\vartheta, m)$ for all $x \in X$, then, locally in $X, P$ has a fundamental solution with the properties required by the general definition of hyperbolicity (H Theorem 9.3.2).

9-702909 Acta mathematica. 124. Imprims lo 13 Avril 1970. 
Homogeneous hyperbolic polynomials. It follows from (3.7') that if $a \in \mathrm{Hyp}(\vartheta)$, then $a(\xi) / a(\vartheta)$ has real coefficients so that a restriction to real polynomials is not essential. All polynomials in the following examples are supposed to be real.

3.10. Examples. The class Hyp $(\vartheta, 0)$ consists of all non-vanishing constants. A linear polynomial $b_{1} \xi+\ldots b_{n} \xi_{n}$ belongs to $\operatorname{Hyp}_{(\vartheta, 1)}$ if and only if $b \vartheta \neq 0$. The quadratic polynomial $a(\xi)=\xi_{1}^{2}-b_{2} \xi_{2}^{2}-\ldots-b_{n} \xi_{n}^{2}$ belongs to $\operatorname{Hyp}(\vartheta, 2)$ with $\vartheta=(1,0, \ldots, 0)$ if and only if $b_{2}, \ldots, b_{n} \geqslant 0$ and it is strongly hyperbolic if and only if $b_{2}, \ldots, b_{n}>0$. More generally, it is easy to see that a quadratic polynomial $a(\xi)$ belongs to Hyp $(\vartheta, 2)$ if and only if $a(\xi) / a(\vartheta)$ has Lorentz signature $1,-1,-1, \ldots, 0, \ldots$ and $\vartheta$ is a time-like vector for $a(\xi) / a(\vartheta)$. It is important to note that Hyp $(\vartheta)$ is closed under multiplication,

$$
a_{1}, a_{2} \in \operatorname{Hyp}(\vartheta) \Rightarrow a_{1} a_{2} \in \operatorname{Hyp}(\vartheta)
$$

and that factors are hyperbolic,

$$
\text { Нyp }(\vartheta) \ni a=a_{1} a_{2} \ldots \Rightarrow a_{1}, a_{2}, \ldots \in \mathrm{Hyp}(\vartheta) .
$$

Note that (3.11) fails in general for $\operatorname{Hyp}^{0}(\vartheta)$. If, e.g. $a \in \mathrm{Hyp}^{0}(\vartheta)$, then $a^{2} \in \mathrm{Hyp}(\vartheta)$ but is not strongly hyperbolic. On the other hand, factors of a strongly hyperbolic polynomial are strongly hyperbolic. Hyperbolic polynomials can also be obtained by polarization. If $a \in \operatorname{Hyp}(\vartheta, m)$ and

$$
a(\xi+t \vartheta)=\sum_{0}^{m} t^{k} a_{k}(\xi)
$$

then $a_{k} \in \operatorname{Hyp}(\vartheta, m-k), 0 \leqslant k \leqslant m$. In fact, $a_{k}(\vartheta)=\left(\begin{array}{c}m \\ k\end{array}\right) a(\vartheta) \neq 0$ and, since the polynomial $t \rightarrow a(\xi+t \vartheta)$ has only real zeros, the same holds for all its derivatives. Since, e.g., $\xi_{1} \xi_{2} \ldots \xi_{n} \in$ Hyp $(\vartheta, n), \vartheta=(1,1, \ldots, 1)$, it follows from this that the elementary symmetric sum $\sum \xi_{1} \xi_{2} \ldots \xi_{k}$ belongs to Hyp $(\vartheta, k)$. Localizations of hyperbolic polynomials are hyperbolic (see Lemma 3.42).

It is obvious that if $a(\xi)$ is real and homogeneous and strongly hyperbolic with respect to $\vartheta$ so is $b(\xi)$ if $b$ is real and homogeneous and sufficiently close to $a$ and degree $b=\operatorname{degree} a$. More generally, we have the following result (W. Nuij 1969) where, temporarily, Hyp $(\vartheta, m)$ and $\operatorname{Hyp}^{0}(\vartheta, m)$ are restricted to real polynomials.

3.13. LЕмма. Let $H(m)$ be the space of non-zero real homogeneous polynomials of degree m. Then $\mathrm{Hyp}^{0}(\vartheta, m)$ is open in $H(m)$ and $\mathrm{Hyp}(\vartheta, m)$ is the part of the closure of $\mathrm{Hyp}^{0}(\vartheta, m)$ where $a(\vartheta) \neq 0$. Both $\mathrm{Hyp}(\vartheta, m)$ and $\mathrm{Hyp}^{0}(\vartheta, m)$ consist of two connected and simply connected pieces determined by the sign of $a(\vartheta)$.

When $a(\xi)$ is a homogeneous polynomial, let $A$ be the complex hypersurface $a(\xi)=0$, 
$\xi \in Z=\mathbf{C}^{n}$ and $\operatorname{Re} A$ its real part. The surface $A$ is said to be hyperbolic when $a \in$ Hyp $(\vartheta)$ for some $\vartheta$. The figure 3 shows the image $\operatorname{Re} A^{*}$ in real projective space of a typical $\operatorname{Re} A$. The picture also shows $\operatorname{Re} B^{*}$ when $b$ is strongly hyperbolic and close to $a$.

The cone $\Gamma(A, \vartheta)$.

3.14. Definition. When $a \in \operatorname{Hyp}(\vartheta)$, let $\Gamma=\Gamma(A, \vartheta)$ be that component of $\operatorname{Re} Z-\operatorname{Re} A$ which contains $\vartheta$ (see figure 3 ).

By the homogeneity of $a, \Gamma(A,-\vartheta)=-\Gamma(A, \vartheta)$. It is clear that $\Gamma$ is an open cone, that $\Gamma=\operatorname{Re} Z$ when $a$ is a constant and that

$$
\Gamma(A, \vartheta)=\bigcap \Gamma\left(A_{k}, \vartheta\right)
$$

when $a=a_{1} a_{2} \ldots$ is a product.

When $a(\eta) \neq 0$, let us factorize

$$
a(\xi+t \eta)=a(\eta) \prod\left(t+\lambda_{k}(\eta, \xi)\right) .
$$

Then $a$ is hyperbolic with respect to $\vartheta$ if and only if all $\lambda_{k}(\vartheta, \xi)$ are real when $\xi$ is real and strongly hyperbolic if and only if these numbers are real and different when $\xi$ is not proportional to $\vartheta$. Further, a real $\xi$ belongs to $\Gamma(A, \vartheta)$ if and only if all $\lambda_{k}(\vartheta, \xi)$ are positive.

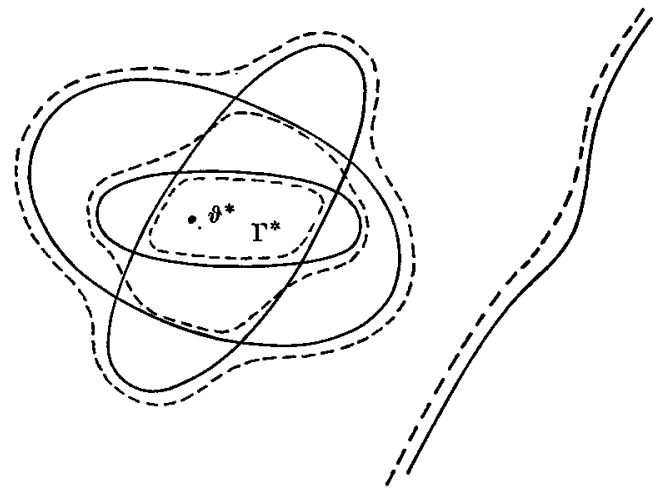

Fig. 3. Picture of $\operatorname{Re} A^{*}, a \in \mathrm{Hyp}_{\mathrm{y}}(\vartheta, 7), n=3$. Every straight line through $\vartheta^{*}$ meets $\operatorname{Re} A^{*}$ in seven real points. The dotted lines indicate $\operatorname{Re} B^{*}$ when $b$ is strongly hyperbolic and close to $a$. $\Gamma^{*}$ is the image in real projective space of $\Gamma(A, \vartheta)$.

Algebraic properties of hyperbolic polynomials. It is obvious that Нyp $(-\vartheta, m)=$ Hyp $(\vartheta, m)$. The following result is less obvious but easy to prove.

3.17. Lemma. hyp $(-\vartheta, m)=\operatorname{hyp}(\vartheta, m)$.

Proof. Let $P \in \operatorname{hyp}(\vartheta, m)$ and let $a=P_{m}$ be the principal part of $P$. Then $a(\vartheta) \neq 0$ and there is an $s_{0}$ such that 


$$
\xi \text { real, } \operatorname{Im} s<s_{0} \Rightarrow P(\xi+s \vartheta) \neq 0 .
$$

Hence, if we factorize

$$
P(\xi+s \vartheta)=a(\vartheta) \Pi\left(s+\mu_{k}(\vartheta, \xi)\right)
$$

(3.18) means that

$$
\xi \text { real } \Rightarrow \operatorname{Im} \mu_{k}(\vartheta, \xi) \geqslant-s_{0}, \forall k
$$

Since $a(-\vartheta)=(-1)^{m} a(\vartheta) \neq 0$, it suffices to show that there is an upper bound to the imaginary parts in $\left(3.18^{\prime}\right)$. Now $\mu(\xi)=\sum \mu_{k}(\vartheta, \xi)$ and hence also $\operatorname{Im} \mu(\xi)$ is a polynomial in $\xi$ of degree one. According to $\left(3.18^{\prime}\right), \operatorname{Im} \mu(\xi)$ is a constant $c$ and also a sum of $m$ terms which are bounded from below by $-s_{0}$. But then no term exceeds $c+(m-1) s_{0}$ and this finishes the proof.

3.20. Lемма. If $P$ belongs to hyp $(\vartheta, m)$, its principal part a belongs to Hyp $(\vartheta, m)$.

Proof. We have, with $\xi$ and $\tau$ real,

$$
a(\xi+t \vartheta)=\lim \tau^{-m} P(\tau \xi+\tau t \vartheta), \quad \tau \rightarrow+\infty,
$$

where all the imaginary parts of the zeros of the polynomials to the right are bounded by $c \tau^{-1}$ where $c$ is a constant. Hence the polynomial $t \rightarrow a(\xi+t \vartheta)$ has only real zeros and, since $a(\vartheta) \neq 0$, there are $m$ of them.

The preceding lemma motivates

3.21. Defintion. When $P \in$ hyp $(\vartheta)$, put $\Gamma(P, \vartheta)=\Gamma(A, \vartheta)$ where $a$ is the principal part of $P$.

Note that $\Gamma(P, \vartheta)$ and $\Gamma(P,-\vartheta)$ are opposite cones. The following simple lemma is basic (Gårding 1950, $\mathrm{H}$ Theorem 5.5.4).

\subsection{Lemma. Let $P \in$ hyp $(\vartheta, m)$ so that $(3.18)$ holds and let $\eta \in \Gamma(P, \vartheta)$. Then}

$$
\xi \text { real, } \operatorname{Im} t \leqslant 0, \operatorname{Im} s<s_{0} \Rightarrow P(\xi+t \eta+s \vartheta) \neq 0 \text {. }
$$

Proof. Since (3.18) holds, the polynomials

$$
t \rightarrow u^{-m} P(\xi+t u \eta+(s+i(1-u)) \vartheta), \quad \operatorname{Im} s<s_{0}, \xi \text { real, } u \geqslant 1
$$

have no real zeros. In particular, they have a constant number of zeros in the lower halfplane. To compute this number note that, as $u \rightarrow \infty$, the polynomials (3.24) tend to 


$$
t \rightarrow a(t \eta-i \vartheta)=a(\vartheta) \prod\left(-i+t \lambda_{k}(\vartheta, \eta)\right),
$$

where $a$ is the principal part of $P$ and (3.16) is used. Now since $\eta \in \Gamma(A, \vartheta)$, all $\lambda_{k}(\vartheta, \eta)$ are positive so that all the zeros of this last polynomial lie in the upper half-plane. Hence all polynomials (3.24) share that property and, putting $u=1$, we have the assertion of the lemma.

3.23. Corollary. If $P \in$ hyp $(\vartheta)$ and $\eta \in \Gamma(P, \vartheta)$, then $P \in$ hyp $(\eta)$. The cone $\Gamma(P, \vartheta)$ is convex.

Proof. If $\eta \in \Gamma(P, \vartheta)$, then $\eta-\varepsilon \vartheta \in \Gamma(P, \vartheta)$ if $\varepsilon>0$ is small enough. Hence, by Lemma 3.22,

$$
P(\xi+t \eta)=P(\xi+t(\eta-\varepsilon \vartheta)+t \varepsilon \vartheta) \neq 0
$$

when $\operatorname{Im} t<\min \left(-s_{0} / \varepsilon, 0\right)$. Also, specializing Lemma 3.22 to the principal part $a$ of $P$, we have

$$
s \geqslant 0, t \geqslant 0, s+t>0 \Rightarrow a(\xi+i t \eta+i s \vartheta) \neq 0,
$$

when $\eta \in \Gamma(A, \vartheta)=\Gamma(P, \vartheta)$. Since $a \in \mathrm{Hyp}(\zeta)$, for any $\zeta \in \Gamma(A, \xi)$, we may here replace $\vartheta$ by such a $\zeta$ and this shows that $\Gamma(P, \xi)$ is convex.

When $P \in$ hyp $(\vartheta)$, the fundamental solution $E(P, \vartheta, x)$ will be constructed as the inverse Fourier-Laplace transform of $P(\xi+i \eta)^{-1}$ where $\eta$ belongs to a certain subset of $-\Gamma(A, \vartheta)$.

The following corollary contains all the necessary information for the construction of such fundamental solutions.

3.24. Corollary. Let $P \in \operatorname{hyp}(\vartheta)$. Then there is an $s_{1}>0$ such that every function

$$
\xi+i n \rightarrow P(\xi+i \eta+i s \vartheta)^{-1}, \quad \varepsilon\left(\operatorname{Re} s-\varepsilon s_{1}\right)>0, \varepsilon= \pm 1,
$$

is holomorphic and bounded when $\xi+i \eta \in \operatorname{Re} Z+\varepsilon i \Gamma(P, \vartheta)$. When $P=a$ is homogeneous, the functions

$$
\xi+i \eta \rightarrow a(\xi+i \eta)^{-1}
$$

are holomorphic in $\operatorname{Re} Z \pm i \Gamma(A, \vartheta)$ and satisfy the inequality

$$
\left|a(s+i \eta)^{-1}\right| \leqslant|a(\eta)|^{-1}
$$

Proof. The functions (3.25) are of course holomorphic where $P$ does not vanish. Replacing $\xi, s$ by $\xi+i \eta$, is in (3.19) gives

$$
P(\xi+i \eta+i s \vartheta)=a(\vartheta) \prod\left(i s+\mu_{k}(\vartheta, \xi+i \eta)\right) .
$$

By Lemma 3.22, $\operatorname{Im} \mu_{k}(\vartheta, \xi+i \eta)$ is bounded from below when $\xi$ is real and $\eta \epsilon-\Gamma=-\Gamma(P, \vartheta)$. 
Hence there is an $s_{0}$ such that the functions (3.25) are bounded when $\xi+i \eta \in \operatorname{Re} Z-i \Gamma$ and Re $s<s_{0}$. Changing $\vartheta$ to $-\vartheta$ and noting that, by Lemma 3.17, $P \in$ hyp $(-\vartheta)$ and that $\Gamma(P,-\vartheta)=-\Gamma(P, \vartheta)$, the first part of the corollary follows. The second part is obvious.

To understand the role of this corollary, the reader should now study Theorem 4.1 before proceeding further. The fundamental solution $E(P, \vartheta, x)$ constructed there has its support in the cone $K=K(P, \vartheta) \subset \operatorname{Re} Z^{\prime}$ dual to $\Gamma(P, \vartheta)$.

Puiseux series. Some of the deeper properties of hyperbolic polynomials result from expanding zeros in Puiseux series. Our next lemma collects material of this kind.

3.27. Lемма. Let $P \in \operatorname{hyp}(\vartheta, m)$ and let $a \in \mathrm{Hyp}(\vartheta, m)$ be the principal part of $P$. Let $\xi, \eta \in \operatorname{Re} Z$. Then the polynomials

$$
\begin{aligned}
s, t \rightarrow a(\xi+t \eta+s \vartheta) \\
s, t \rightarrow P(\xi+t \eta+s \vartheta)
\end{aligned}
$$

can be factorized in the form

$$
\begin{aligned}
& a(\xi+t \eta+s \vartheta)=a(\vartheta) \prod_{1}^{m}\left(s+\lambda_{k}(\vartheta, \xi+t \eta)\right) \\
& P(\xi+t \eta+s \vartheta)=a(\vartheta) \prod_{1}^{m}\left(s+\mu_{k}(\vartheta, \xi+t \eta)\right),
\end{aligned}
$$

where the functions

$$
R \ni t \rightarrow \lambda_{k}(\vartheta, \xi+t \eta)
$$

are real and analytic with simple poles at $\infty$ so that

$$
\lambda_{k}(\vartheta, \xi+t \eta)=t \lambda_{k}(\vartheta, \eta)+O(1), \quad t \rightarrow \infty
$$

When $\eta \in \Gamma=\Gamma(A, \vartheta)$, their derivatives are positive, when $\eta \in \bar{\Gamma}$ they are non-negative. The functions

$$
R \ni t \rightarrow \mu_{k}(\vartheta, \xi+t \eta)
$$

can be labelled so that, for large $t$,

$$
\mu_{k}(\vartheta, \xi+t \eta)=t \lambda_{k}(\vartheta, \xi)+O(1) .
$$

Proof. Close to any real $t_{0}$, the functions (3.30) can be developed into convergent Puiseux series

$$
\lambda_{k}(\vartheta, \xi+t \eta)=\lambda_{k}\left(\vartheta, \xi+t_{0} \eta\right)+c_{k}\left(t-t_{0}\right)^{r_{k}}(1+o(1)),
$$


where $c_{k} \neq 0$ and $r_{k}>0$ is rational. All the branches of these series being real when $t$ is real implies that $r_{k}$ is an integer and also that the series are power series in $t-t_{0}$ with real coefficients. Varying $t_{0}$ and making an analytic continuation proves the first statement of the lemma. Taking $t$ large and noting that

$$
t \rightarrow \infty \Rightarrow t^{-m} P(\xi+t \eta+t s \vartheta) \rightarrow a(\eta+s \vartheta),
$$

where the convergence refers to polynomials in $s$, it follows that

$$
\mu_{k}(\vartheta, \xi+t \eta)=t \lambda_{k}(\vartheta, \eta)+o(t), \quad 1 \leqslant k \leqslant m
$$

if the $\mu$ 's are labelled suitably. Now the left sides can be developed in Puiseux series in descending powers of $t^{1 / m}$ starting with $t$. Since by the hyperbolicity of $P$, their imaginary parts are bounded, no series can contain a positive power of $t^{1 / m}$ except the first term of (3.35) and hence (3.31) follows. When this reasoning is applied to the real functions (3.32), (3.33) follows. Finally, if $\eta \in \Gamma=\Gamma(A, \vartheta)$, then, since $\Gamma$ is convex, $\operatorname{Im}(t \eta+s \vartheta)$ belongs to $\Gamma$ when $\operatorname{Im} t \geqslant 0, \operatorname{Im} s \geqslant 0, \operatorname{Im}(t+s)>0$ and hence, by Lemma 3.22,

$$
\operatorname{Im} t>0 \Rightarrow \operatorname{Im} \lambda_{k}(\vartheta, \xi+t \eta)>0 .
$$

This is possible only if $r_{k}=1$ and $c_{k}>0$ in (3.34) and hence the functions (3.30) have positive derivatives. A slight modification of this argument shows the derivatives are $\geqslant 0$ when $\eta \in \bar{\Gamma}$.

\section{Multiplicities and localization}

3.36. Definition. Let $P(\xi)$ be a polynomial of degree $m \geqslant 0$ and develop $t^{m} P\left(t^{-1} \xi+\zeta\right)$ in ascending powers of $t$,

$$
t^{m} P\left(t^{-1} \xi+\zeta\right)=t^{p} P_{\xi}(\zeta)+O\left(t^{p+1}\right)
$$

where $P_{\xi}(\zeta)$ is the first coefficient that does not vanish identically in $\zeta$. The number $p=m_{\xi}(P)$ is called the multiplicity of $\xi$ relative to $P$, the polynomial $\zeta \rightarrow P_{\xi}(\zeta)$, the localization of $P$ at $\xi$.

Note that if $P=P_{0}+\ldots+P_{m}$ is a decomposition of $P$ into homogeneous parts, $P_{k}(\lambda \xi)=$ $\lambda^{k} P_{k}(\xi)$, then (3.37) reads

$$
\sum t^{m-k} P_{k}(\xi+t \zeta)=t^{p} P_{\xi}(\zeta)+O\left(t^{p+1}\right)
$$

In particular, when $P=a$ is homogeneous,

$$
a(\xi+t \zeta)=t^{p} a_{\xi}(\zeta)+O\left(t^{p+1}\right) .
$$

It is obvious that 


$$
\begin{gathered}
m_{\xi}(P Q)=m_{\xi}(P)+m_{\xi}(Q) \\
(P Q)_{\xi}(\zeta)=P_{\xi}(\zeta) Q_{\xi}(\zeta)
\end{gathered}
$$

when $P$ and $Q$ are (non-vanishing) polynomials.

The concept of localization extends to non-zero rational functions $f=Q / P$,

$$
t^{m} f\left(t^{-1} \xi+\zeta\right)=t^{p} f_{\xi}(\zeta)+O\left(t^{p+1}\right)
$$

Here the right side is a formal power series in $t$ whose coefficients are rational functions of $\zeta$, the localization $f_{\xi}$ is the first non-vanishing coefficient, $m=m(f)=m(Q)-m(P)$ is the degree of $f$ and $p=m_{\xi}(f)$ is the multiplicity of $f$ at $\xi$. One has $f_{\xi}=Q_{\xi} / P_{\xi}$ and $m_{\xi}(f)=$ $m_{\xi}(Q)-m_{\xi}(P)$.

Examples. Let $a$ be the principal part of $P$. If $\xi=0$, then $P_{\xi}(\zeta)=P(\zeta)$. If $a(\xi) \neq 0$, then $P_{\xi}(\zeta)=a(\xi)$ is a non-zero constant. If $a(\xi)=0$ but $\operatorname{grad} a(\xi) \neq 0$, then

$$
P_{\xi}(\zeta)=\operatorname{grad} a(\xi) \zeta+\text { const }
$$

is a polynomial of degree 1 . When $P$ is strongly hyperbolic, these examples exhaust the possibilities for $P_{\xi}$ with $\xi \neq 0$. When $P$ is not strongly hyperbolic, the degree of such a $P_{\xi}$ may of course exceed I. If $P=a$ is homogeneous, then $a_{\xi}(\zeta)=0$ defines the tangent cone $A_{\xi}$ of $A: a(\xi)=0$ at $\xi$.

Localizations of non-homogeneous polynomials in the sense given above have been used by Hörmander (1969) in a study of singularities of fundamental solutions for arbitrary differential operators.

Our next lemma shows that localizations at real points of hyperbolic polynomials are hyperbolic.

3.42. Lem м. Let $P \in$ hyp $(\vartheta)$ with principal part $a$ and let $\xi \in \operatorname{Re} Z$. Then

$$
\begin{gathered}
m_{\xi}(P)=m_{\xi}(a) \\
a_{\xi} \text { is the principal part of } P_{\xi} \\
P_{\xi} \in \text { hyp }(\vartheta), a_{\xi} \in \operatorname{Hyp}(\vartheta) \\
A_{\xi} \text { depends only on the double ray } \dot{R}_{\xi} \\
\Gamma\left(A_{\xi}, \vartheta\right) \supset \Gamma(A, \vartheta) .
\end{gathered}
$$

Proof. Let us first consider $a$. Putting $s=0$ in (3.28) and using the differentiability in (3.30) we get 


$$
a(\xi+t \eta)=a(\vartheta) \prod_{1}^{m}\left(\lambda_{k}(\vartheta, \xi)+t c_{k}+O\left(t^{2}\right)\right)
$$

where $t$ is small and all $c_{k}>0$ when $\eta \in \Gamma=\Gamma(A, \vartheta)$. Since $\Gamma$ is open, a comparison with (3.39) shows that

$$
\eta \in \Gamma \Rightarrow a_{\xi}(\eta) \neq 0
$$

and that precisely $p=m_{\xi}(a)$ of the numbers $\lambda_{k}(\vartheta, \xi)$ vanish. Now, by the preceding lemma,

$$
t^{m} P\left(t^{-1} \xi+\eta\right)=a(\vartheta) \prod t \mu_{k}\left(t^{-1} \xi+\eta\right)=a(\vartheta) \Pi\left(\lambda_{k}(\vartheta, \xi)+O(t)\right)
$$

when $\eta$ is real. Hence

$$
t^{m} P\left(t^{-1} \xi+\eta\right)=O\left(t^{p}\right)
$$

and this shows that $m_{\xi}(P) \geqslant p$. Hence, developing the left side of (3.37) in a Taylor series around $t^{-1} \xi$, we get

$$
t^{m} P\left(t^{-1} \xi+\eta\right)=\sum_{p}^{m} t^{k} f_{k}(\eta)
$$

where the $f_{k}$ are polynomials in $\eta$ of degree $\leqslant k$ depending on $\xi$ as a parameter. Replacing $t$ by $t s^{-1}$ and $\eta$ by $s \eta$ and letting $s \rightarrow \infty$ this gives

$$
t^{m} a\left(t^{-1} \xi+\eta\right)=\sum_{p}^{m} t^{k} f_{k}^{(k)}(\eta)
$$

where

$$
f_{k}^{(k)}(\eta)=\lim s^{-k} f_{k}(s \eta), \quad s \rightarrow \infty,
$$

is the homogeneous part of $f_{k}$ of degree $k$. A comparison with (3.37) now shows that $f_{p}^{(p)}(\eta)=$ $a_{\xi}(\eta)$. Hence $f_{p}(\eta)$ does not vanish identically and this means that $P_{\xi}(\eta)=f_{p}(\eta)$ has the principal part $a_{\xi}(\eta)$. This proves (3.43) and (3.44). By the definition of $P_{\xi}$ and $p=m_{\xi}(P)$,

$$
R \ni t \rightarrow 0 \Rightarrow t^{m-p} P\left(t^{-1} \xi+\eta+s \vartheta\right)=P_{\xi}(\eta+s \vartheta) .
$$

Since $P \in$ hyp $(\vartheta)$, there is an $s_{0}$ such that the polynomials

$$
s \rightarrow t^{m-p} P\left(t^{-1} \xi+\eta+s \vartheta\right)
$$

have no zeros $s$ with $\operatorname{Im} s<s_{0}$ when $\eta$ is real. According to (3.50), the polynomials $s \rightarrow$ $P_{\xi}(\eta+s \vartheta)$ have the same property. Since (3.44) holds and, by $(3.48), a_{\xi}(\vartheta) \neq 0$, this proves (3.45). Finally, (3.47) is a consequence of (3.48) and the definition of $\Gamma\left(A_{\xi}, \vartheta\right)$ and (3.46) is a simple consequence of (3.39) and the homogeneity of $a$, one has

when $0 \neq \lambda \in R$.

$$
a_{\lambda \xi}(\eta)=\lambda^{m-\eta} a_{\xi}(\eta)
$$

In passing we shall now prove a quantitative result, to be used later.

3.51. Lemma. Let $P \in \operatorname{hyp}(\vartheta), p=m_{\xi}(P), \xi \in \operatorname{Re} Z$ and choose $s_{0}$ such that $P(\eta+s \vartheta) \neq 0$ 
when $|\operatorname{Im} s|>s_{0}$. Then there exist positive numbers $c$ and $N$ depending only on $s$ and $\xi$ such that, if $\zeta$ is real,

$$
\begin{aligned}
0 \leqslant t \leqslant 1,|\operatorname{Im} s| & >s_{0} \Rightarrow\left|t^{m-p} P\left(t^{-1} \xi+\zeta+s \vartheta\right)\right| \geqq c(1+|\zeta|)^{-N} \\
t & \rightarrow 0 \Rightarrow t^{m-p} P\left(t^{-1} \xi+\zeta+s \vartheta\right) \rightarrow P_{\xi}(\zeta+s \vartheta)
\end{aligned}
$$

Proof. Fixing $s$ and $\xi$, let us put

$$
R(t, \zeta)=\left|t^{m-p} P\left(t^{-1} \xi+\zeta+s \vartheta\right)\right|^{2}
$$

Then $R$ is a polynomial in $t$ and $\eta$ and, since $R(0, \zeta)=\left|P_{\xi}(\zeta+s \vartheta)\right|^{2}>0$, then

$$
\varphi(\tau)=\inf _{t^{2}+|\zeta| \leqslant \tau^{2}+1} R(t, \zeta) \leqslant \inf _{0<t \leqslant 1,|\zeta|<\tau} R(t, \zeta)
$$

is positive for all $\tau$. Now by the well-known Seidenberg-Tarski theorem (see Lemma 2.1 of the Appendix in $\mathrm{H}$ ), for large $\tau, \varphi(\tau)$ is not less than a positive constant times some negative power $\tau$. This proves the lemma.

Lineality and reduced dimension. A hyperbolic polynomial $P(\xi), \xi \in Z$, may depend on less than $\operatorname{dim} Z$ variables. The lineality $L(P)$ of $P$ in $Z$ is, by definition, the space of $\xi \in Z$ such that $P(t \xi+\zeta)=P(\zeta)$ identically in $\zeta, t$ or, equivalently, $P_{\xi}(\zeta)=P(\zeta)$ for all $\zeta$, i.e. $m_{\xi}(P)=$ degree $P$. It is clear that $L(P)$ is a linear space and that

$$
L(P Q)=L(P) \cap L(Q)
$$

when $P, Q$ are not identically zero. Hence, writing $P$ as a product of irreducible factors, we see that if $P \neq 0$, then $L(P)$ depends only on the complex surface $P(\xi)=0$. When $P=a$ is homogeneous we shall therefore write $L(A)=L(a)$ where now $A$ is the complex surface $a(\xi)=0$. It is obvious that $P$ is a polynomial $P^{\prime}$ on the quotient $Z / L(P)$ and that the lineality of $P^{\prime}$ in the quotient vanishes. The dimension of the quotient, i.e. the codimension of $L(P)$ in $Z$, will be called the reduced dimension of $P$ and denoted by $n(P)$. When $P=a$ is homogeneous we also write $n(A)=n(a)$. A polynomial whose lineality vanishes is said to be complete. We have

3.52. Lемма. Let $P \in$ hyp $(\vartheta)$ and let a be the principal part of $P$. Then $L(P)=L(A)$, $n(P)=n(A)$ and $\operatorname{Re} L(A)$ equals the edge of $\Gamma=\Gamma(A, \vartheta)$, i.e. $\operatorname{Re} L(A)$ consists of all $\xi \in \operatorname{Re} Z$ such that

$$
\Gamma+R \xi=\Gamma
$$

If $a_{\xi}$ is the localization of a at $\xi \in Z$, then $\xi \in L\left(A_{\xi}\right)$. 
Proof. By Lemma 3.42, $m_{\xi}(P)=m_{\xi}(a)$ when $\xi$ is real. In particular, $\operatorname{Re} L(P)=$ $\operatorname{Re} L(A)$ so that, clearly, $L(P)=L(A)$. Let us factorize

$$
a\left(s \eta+\eta^{\prime}+t \xi\right)=a(\eta) \prod_{1}^{m}\left(s+\lambda_{k}\left(\eta, \eta^{\prime}+t \xi\right)\right), \quad \eta \in \Gamma .
$$

By the definition of $\Gamma, \xi$ satisfies (3.53) if and only if

$$
\lambda_{k}\left(\eta, \eta^{\prime}+t \xi\right)>0, \forall \eta, \eta^{\prime} \in \Gamma, \quad \forall t \in R .
$$

If $\xi \in \operatorname{Re} L(A)$, then $\lambda_{k}\left(\eta, \eta^{\prime}+t \xi\right)=\lambda_{k}\left(\eta, \eta^{\prime}\right)>0$ and the condition holds. Conversely, if (3.54) holds, dividing by $t$ and letting $t \rightarrow \infty$, we get $\lambda_{k}(\eta, \xi)=0$ for all $\eta \in \Gamma$ so that also $a(\eta+t \xi)=a(\eta)$ for all $t$ and $\eta \in \Gamma$. But then, since $\Gamma$ is open, $\xi \in \operatorname{Re} L(A)$. By the definition of $a_{\xi}$,

$$
a(\xi+t(\zeta+s \xi))=a_{\xi}(\zeta+s \xi) t^{p}+O\left(t^{p+1}\right),
$$

where $p=m_{\xi}(a)$. But the left side also equals

$$
(1+t s)^{m} a\left(\xi+(1+t s)^{-1} t \zeta\right)=a_{\xi}(\zeta) t^{p}(1+t s)^{m-p}+O\left(t^{p+1}\right)
$$

Identifying the coefficients of $t^{p}$ of the right sides proves the last statement of the lemma.

The propagation cone. When $P \in$ hyp $(\vartheta)$, we know that the convex hull of the support of the fundamental solution $E(P, \vartheta, \cdot)$ is the cone dual to $\Gamma(P, \vartheta)=\Gamma(A, \vartheta)$ where $a$ is the principal part of $P$. This cone will be called the propagation cone, its formal definition being

$$
K(P, \vartheta)=K(A, \vartheta)=\left\{x ; x \in \operatorname{Re} Z^{\prime}, x \Gamma(A, \vartheta) \geqslant 0\right\}
$$

We state some of its properties

3.56. Lemma. Let $a \in \operatorname{Hyp}(\vartheta)$. Then

a) $K=K(A, \vartheta)$ is a proper closed convex cone with its vertex at the origin and

$$
\Gamma=\Gamma(A, \vartheta)=\{\xi ; \xi \in \operatorname{Re} Z, \xi \dot{K}>0\}, \dot{K}=K-\{0\} .
$$

b) $K$ is contained in and spans the orthogonal complement

$$
L^{0}(A)=\left\{x ; x \in \operatorname{Re} Z^{\prime}, x L(A)=0\right\}
$$

of the lineality of $a$. Hence $\operatorname{dim} K=n(A)$.

c) The interior of $K$ relative to $L^{0}(A)$ consists of all

$$
\nabla a(\xi), \quad \xi \in \Gamma
$$


and also of all

$$
\nabla \nabla_{\eta} \log a(\xi)^{-1}, \quad \xi \in \Gamma
$$

Here $\nabla=\operatorname{grad}, \nabla_{\eta}=(\eta, \mathrm{grad}), \eta$ is any vector in $\Gamma$ and $a$ is normalized so that $a(\vartheta)>0$.

Proof. a) and b) follow from the preceding lemma and our earlier remarks about the duality of cones. In view of b), it suffices to prove c) when $a$ is complete. We know then that if $x \in \stackrel{\circ}{K}$, the intersection of $\bar{\Gamma}$ with any half-space $x \xi \leqslant c$ is compact. In particular, if $B$ is a closed part of $\Gamma$, the infimum $c$ of $x \xi$ on $B$ is positive and the hyperplane $x \xi=c$ touches $B$ at some point $\eta=\eta(x) \in B$. Taking $B$ to be the hypersurface $a(\xi)=1, \xi \in \Gamma$ and noting that, since $a$ has non-zero homogeneity, $\nabla a(\xi)$ does not vanish in $\Gamma$, we see that every $x$ in $\stackrel{\circ}{K}$ is proportional to some $\nabla a(\eta), \eta \in B$. Clearly, the proportionality constant is positive and this proves that every $x$ in $\dot{K}$ has the form (3.57). Actually, $B$ is strictly convex (see Gårding (1959)) so that the parametrization is unique. Similarly, letting $B$ be the hypersurface $F(\xi)=\nabla_{\eta} \log a(\xi)^{-1}=-1, \xi \in \Gamma$, it follows that every $x$ in $\dot{K}$ has the form (3.58). In fact, factorizing

$$
a(\xi+t \eta)=a(\eta) \prod_{1}^{m}\left(t+\lambda_{k}(\eta, \xi)\right)
$$

all $\lambda_{k}$ are positive and

$$
F(\xi)=-\sum_{1}^{m} \lambda_{k}(\eta, \xi)^{-1}
$$

so that $B$ is not empty. Since $F$ has homogeneity $-1, \nabla F \neq 0$ in $\Gamma$. It remains to show that all vectors (3.57) and (3.58) are in $\dot{K}$. Writing (3.59) in the form

$$
a(\xi+t \eta)=a(\xi) \Pi\left(1+t \lambda_{k}(\xi, \eta)\right)
$$

we have, if $\xi \in \Gamma$ and $\eta \in \operatorname{Re} Z$

$$
\nabla_{\eta} a(\xi)=a(\xi)\left(\lambda_{1}(\xi, \eta)+\ldots+\lambda_{m}(\xi, \eta)\right)
$$

Now, if $0 \neq \eta \in \bar{\Gamma}$, all $\lambda_{k}$ are non-negative and at least one is positive. This shows that the linear form $\eta \rightarrow \nabla_{\eta} a(\xi)$ is positive on $\bar{\Gamma}-\{0\}$ and hence $\nabla a(\xi) \in \dot{K}$. Next, let $\eta, \xi \in \Gamma$ and $\zeta \in \operatorname{Re} Z$. Replacing $\xi$ in (3.60) by $\xi+t \zeta$ and taking the derivative with respect to $t$ at $t=0$, we have

$$
\nabla_{\zeta} F(\xi)=\sum \lambda_{k}(\eta, \xi)^{-2} c_{k}(\eta, \xi, \zeta)
$$

where

$$
\lambda_{k}(\eta, \xi+t \zeta)=\lambda_{k}(\eta, \xi)+t c_{k}(\eta, \xi, \zeta)+O\left(t^{2}\right) \text {. }
$$

By Lemma 3.27, all $c_{k}$ are $\geqslant 0$ when $\zeta \in \bar{\Gamma}$ and hence also $\nabla_{\zeta} F(\xi) \geqslant 0$. If all $c_{k}$ vanish, then 


$$
a(\xi+t \zeta)=a(\eta) \prod_{1}^{m} \lambda_{k}(\eta, \xi+t \zeta)=a(\eta) \prod_{1}^{m}\left(\lambda_{k}(\eta, \xi)+O\left(t^{2}\right)\right)
$$

so that $\nabla_{\zeta} a(\xi)=0$. But then, by a previous argument, $\zeta$ has to vanish. Hence the linear form $\zeta \rightarrow \nabla_{\zeta} F(\xi)$ is positive on $\bar{\Gamma}-\{0\}$ and this shows that all gradients (3.58) are in $\dot{K}$. The proof is finished.

Local cones. The wave front surface. We shall introduce the cones and linealities associated with the localizations of a hyperbolic polynomial.

3.61. Definition. When $P \in$ hyp $(\vartheta), \xi \in \operatorname{Re} Z$, put

$$
\Gamma_{\xi}(P, \vartheta)=\Gamma\left(P_{\xi}, \vartheta\right), \quad K_{\xi}(P, \vartheta)=K\left(P_{\xi}, \vartheta\right), \quad L_{\xi}(P)=L\left(P_{\xi}\right), \quad n_{\xi}(P)=\operatorname{codim} L_{\xi}(P)
$$

Examples. Let $a$ be the principal part of $P$. When $\xi=0$ we have the uninteresting case $P_{\xi}=P$. When $a(\xi) \neq 0$, then $P_{\xi}(\zeta)=a(\xi)$ is a constant and $\Gamma_{\xi}=\operatorname{Re} Z, K_{\xi}=\{0\}, n_{\xi}=0$. When $a(\xi)=0$ but $\nabla a(\xi) \neq 0$, then $a_{\xi}(\zeta)=\nabla_{\xi} a(\xi)=\sum \zeta_{k} \partial a(\xi) / \partial \xi_{k}$ so that $\Gamma_{\xi}$ is the half-space $a_{\xi}(\vartheta)^{-1} a_{\xi}(\zeta)>0, n_{\xi}=1$ and $K_{\xi}$ is the half-ray spanned by $a_{\xi}(\vartheta)^{-1} \nabla a(\xi)$. When $P$ is strongly hyperbolic and $\xi \neq 0$, these examples are exhaustive. When $P$ is not strongly hyperbolic, $\Gamma_{\xi}$ may be smaller than a half-space and $K_{\xi}$ larger than a half-ray and $n_{\xi}>1$. Note that $\Gamma_{\xi}=\Gamma, K_{\xi}=K$ when $\xi \in L(P)$.

By Lemma 3.42, $\Gamma_{\xi}(P, \vartheta) \supset \Gamma(P, \vartheta)$ and hence also $K_{\xi} \subset K(P, \vartheta)$ for all real $\xi$. The local propagation cones $K_{\xi}$ generate the wave front surface

$$
W=W(P, \vartheta)=W(A, \vartheta)=\bigcup K_{\xi}(P, \vartheta), \quad \xi \neq 0,
$$

which will be dealt with in detail later. Its role as the carrier of the singularities of the fundamental solution $E=E(P, \vartheta, x)$ is explained in the introduction. In particular, it will turn out that the restriction of $E$ to $K-W$ is holomorphic. When $L(P) \neq 0$, then $W=K$ so that $W$ is not an interesting object in that case. When $L(P)=0$, i.e. $P$ is a complete polynomial, then, as we shall see later, $W$ is a closed subset of $K$ containing the boundary of $K$ and contained in an algebraic hypersurface. The same would be true in general with a different definition of $W$, viz. as $\bigcup K_{\xi}(P, \vartheta)$ for $\xi \bar{\epsilon} L(P)$.

Hyperbolic polynomials with a given principal part. Suppose that $a \in \mathrm{Hyp}(\vartheta, m)$ is strongly hyperbolic and that the degree of $b$ is less than $m$. Then

$$
\sup _{\xi}\left|\left(b a^{-1}\right)(\xi+s \vartheta)\right|=O\left(|\operatorname{Im} s|^{-1}\right), \quad \xi \text { real, } \operatorname{Im} s \rightarrow \infty
$$

In fact, it suffices to prove this when $\xi$ is restricted to some complement $Y$ of $R \vartheta$ in $\operatorname{Re} Z$. By partial fractions, 


$$
\left(b a^{-1}\right)(\xi+s \vartheta)=a(\vartheta)^{-1} \sum B_{k}(\xi)\left(s+\lambda_{k}\right)^{-1},
$$

where the $\lambda_{k}=\lambda_{k}(\vartheta, \xi)$ as defined by $(3.16)$ are real and

$$
B_{k}(\xi)=b\left(\xi-\lambda_{k} \vartheta\right) / \prod_{j \neq k}\left(\lambda_{k}-\lambda_{j}\right)
$$

Since $a$ is strongly hyperbolic, $\lambda_{k}(\vartheta, \xi) \neq \lambda_{j}(\vartheta, \xi)$ when $j \neq k$ and $0 \neq \xi \in Y$ and an easy argument shows that the $B_{k}$ are bounded for large $\xi$ so that (3.62) follows.

It is clear that (3.62) implies that $(a+b)(\xi+s \vartheta) \neq 0$ when $\xi$ is real and $\operatorname{Im} s$ is large enough and hence $a+b \in$ hyp $(\vartheta)$. In the general case, (3.62) characterizes every $b$ of degree less than $a$ such that $a+b \in$ hyp $(\vartheta)$. We have the following result due to Leif Svensson (1970).

3.63. Тнаов $a+b \in$ hyp ( $(9)$ if and only if (3.62) holds.

Later we shall use this theorem, but the proof is rather long and will not be reproduced here.

\section{Fundamental solutions of scalar hyperbolic operators. Localization. Analytic continuation}

We shall first construct fundamental solutions of scalar hyperbolic operators and state their simplest properties.

\subsection{Thв OR EM. Let $P \in$ hyp $(\vartheta)$. Then}

$$
E(P, x)=E(P, \vartheta, x)=(2 \pi)^{-n} \int_{R_{\theta} Z} P(\xi+i \eta)^{-1} e^{i x(\xi+i \eta)} d \xi,
$$

where $\eta \epsilon-s \vartheta-\Gamma(P, \vartheta)$ with s large enough, is the fundamental solution of $P$ with support in $K=K(P, \vartheta)$. No smaller convex cone than $K$ contains the support of $E(P, x)$. When $P=a$ is homogeneous, then also

$$
E(a, \vartheta, x)=(2 \pi)^{-n} \int_{\mathrm{Re} Z} a_{-}(\xi)^{-1} e^{i x \xi} d \xi
$$

where

$$
a_{-}(\xi)^{-1}=\lim a(\xi+i t \eta)^{-1}, \quad t \downarrow 0, \quad \eta \in-\Gamma(A, \vartheta) .
$$

When $P=a+b$ where $a$ is the principal part of $P$, then 


$$
E(P, \vartheta, x)=\sum_{0}^{\infty}(-1)^{k} b(D)^{k} E\left(a^{k+1}, \vartheta, x\right)
$$

where the series converges in the distribution sense.

If $t>0$, then

$$
E(a, \vartheta, t x)=t^{m-n} E(a, \vartheta, x)
$$

while, if $t \downarrow 0$,

$$
t^{n-m} E(P, \vartheta, t x) \rightarrow E(a, \vartheta, x)
$$

in the distribution sense. If the coordinates $x_{1}, \ldots$ are chosen so that the first $q=n(P)$ axes span the orthogonal complement of $L(P)$, then

$$
E(P, \vartheta, x)=E\left(P^{\prime}, \vartheta^{\prime}, x^{\prime}\right) \delta\left(x^{\prime \prime}\right),
$$

where $x^{\prime}=\left(x_{1}, \ldots, x_{q}\right), x^{\prime \prime}=\left(x_{q+1}, \ldots\right), P^{\prime}=P\left(D^{\prime}\right)$.

Note. $P^{\prime}\left(\xi^{\prime}\right)$ is the restriction of $P(\xi)$ to a complement of $L(P)$ and it is a complete polynomial. The formula (4.8) shows that it suffices to compute fundamental solutions for complete hyperbolic operators in order to have them for all hyperbolic operators.

Proof. Note that (4.2) has to be understood as the corresponding equality when both sides are applied to a test function $g \in C_{0}(\operatorname{Re} Z)$,

$$
(E, \breve{g})=(2 \pi)^{-n} \int_{R \in Z} P(\xi+i \eta)^{-1} \mathcal{F} g(\xi+i \eta) d \xi .
$$

With this understanding, the first part of the theorem is a consequence of Theorem 2.6 and Corollary 3.24. The same applies to (4.3). In fact, we only have to verify that $E$ is a fundamental solution, but this follows from (4.2') if we replace $E$ by $P(D) E$. Then, by (2.2),

$$
(P(D) E, \check{g})=\left(E,(P(D) g)^{\nu}\right)=(2 \pi)^{-n} \int \mathcal{F} g(\xi+i \eta) d \xi=g(0) .
$$

Next, let $K_{0} \subset K$ be a closed convex cone containing the support of $E(P, x)$ and choose an $\eta \in \operatorname{Re} Z$ such that $\eta x>0$ on $K_{0}$. Then, by the first part of Theorem 3.5, $P \in$ hyp $(\eta)$ so that, by Lemma 3.20, $a \in \mathrm{Hyp}(\eta)$ where $a$ is the principal part of $P$. It follows that $a(\eta) \neq 0$ when $\eta$ belongs to the interior $\Gamma_{0}$ of the dual cone of $K_{0}$. Now, since $K_{0} \subset K$, we have $\Gamma_{0} \supset \Gamma=\Gamma(P, \vartheta)$. But $a(\eta)$ vanishes on the boundary of $\Gamma$ and hence $\Gamma_{0}=K$ so that $K_{0}=K$. To prove (4.5) note that, formally, (4.5) results from $\left(4.2^{\prime}\right)$ by an expansion in a geometric series 


$$
P^{-1}(\xi+i \eta)=\sum_{0}^{\infty}(-1)^{k} b(\xi+i \eta)^{k} a(\xi+i \eta)^{-k-1}
$$

The convergence of this series when $\eta=s \vartheta$ with $s$ large negative results from Theorem 3.63. Moreover, the convergence is uniform in $\xi$. Hence (4.5) follows. (4.6) is an obvious consequence of (4.2) when $P=a$ is homogeneous of degree $m$. To prove (4.7) observe that $t^{n-m} E(P, \vartheta, t x)$ is the fundamental solution of $P_{t}(D)=t^{m} P\left(t^{-1} D\right)$ and that $P_{t}(D)=a(D)+$ $b_{t}(D)$ where $b_{t}(D)=t^{m} b\left(t^{-1} D\right)$. By Theorem 3.60,

$$
t^{m} b\left(t^{-1} \xi-t^{-1} i s \vartheta\right) a(\xi-i s \vartheta)=b\left(t^{-1} \xi-t^{-1} i s \vartheta\right) a\left(t^{-1} \xi-i s t^{-1} \vartheta\right)=O\left(\operatorname{Re} s t^{-1}\right)
$$

when $s t^{-1}$ is large. Hence, fixing $s$ and letting $t \downarrow 0$, the series (4.5) for $P_{t}$ converges in the distribution sense to its first term. Finally, (4.8) is an immediate consequence of (4.2').

Localization of fundamental solutions. When $P \in$ hyp $(\vartheta)$ and $P_{\xi} \in$ hyp $(\vartheta)$ is the localization of $P$ at $\xi \in \operatorname{Re} Z$, consider the corresponding fundamental solution

$$
E_{\xi}=E_{\xi}(P, \vartheta, x)=E\left(P_{\xi}, \vartheta, x\right)
$$

According to what we have just proved,

$$
\mathrm{S}\left(E_{\xi}\right) \subset K_{\xi}=K\left(P_{\xi}, \vartheta\right) \subset K=K(P, \vartheta)
$$

Let $a$ be the principal part of $P$. As remarked before, $K_{\xi}=\{0\}$ when $a(\xi) \neq 0$ and, when $a(\xi)=0, \operatorname{grad} a_{\xi} \neq 0$, then $P_{\xi}$ is a polynomial of degree 1 and $K_{\xi}$ is just the positive half of the normal (grad $a(\xi) \cdot \vartheta)^{-1} \operatorname{grad} a(\xi)$ to $A: a(\zeta)=0$, at $\xi$. The following theorem, where SS denotes 'the singular support of' shows that in a sense $E_{\xi}$ is a localization of $E=E(P, \vartheta, x)$. The idea of localizing fundamental solutions by taking limits like (4.11) is due to Lars Hörmander; our next theorem is just a special case of a general theory (Hörmander (1969)).

4.10. Looalization Theorem. Let $P \in \operatorname{hyp}(\vartheta, m)$, let $E, \xi, E_{\xi}$ be as above and let $p=m_{\xi}(P)$ be the multiplicity of $\xi$ relative to $P$. Then

$$
R \ni t \rightarrow \infty \Rightarrow t^{m-p} e^{-i t x \xi} E(x) \rightarrow E_{\xi}(x)
$$

in the distribution sense and

$$
\xi \neq 0 \Rightarrow \mathrm{S}\left(E_{\xi}\right) \subset \mathrm{SS}(E) .
$$

Proof. Letting $\zeta$ be a new variable of integration in (4.2) we have

$$
E_{t}(x)=e^{-i t x \xi} E(P, x)=(2 \pi)^{-n} \int_{\mathrm{R} \theta Z} P(t \xi+\zeta+i \eta)^{-1} e^{i x(\zeta+i \eta)} d \zeta .
$$

Hence, by $\left(4.2^{\prime}\right)$, 


$$
\left(t^{m-p} E_{t}, \breve{g}\right)=(2 \pi)^{-n} \int_{\mathrm{Re} Z}\left(t^{p-m} P(t \xi+\zeta+i \eta)\right)^{-1} \mathcal{F} g(\zeta+i \eta) d \zeta .
$$

Putting $\eta=-s \vartheta$ with $s$ large negative, Lemma 3.51 shows that the right side tends to

$$
(2 \pi)^{-n} \int P_{\xi}(\zeta+i \eta)^{-1} \mathcal{F} g(\zeta+i \eta) d \zeta=\left(E_{\xi}, \check{g}\right) .
$$

This proves (4.11). Next, let $V$ be the complement of $\operatorname{SS}(E)$. If $g \in C_{0}(V)$ and $\xi \neq 0$, then, obviously,

$$
\int t^{m-p} e^{-i t t \xi} E(P, x) g(x) d x
$$

tends to zero as $t \rightarrow \infty$. Hence $\mathrm{S}\left(E_{\xi}\right)$ is contained in the complement of $V$ and this finishes the proof.

It follows from $(4,10)$ that

$$
\mathrm{SS}(E) \supset \bigcup_{\xi \neq 0} \mathrm{~S}\left(E_{\xi}\right)
$$

So far we have been unable to find a case where this inclusion is proper.

The localization theorem extends to derivatives of the fundamental solution. The precise statement is as follows, the proof is the same as before.

4.10'. Localization Theorma. Let $E(P)=E(P, \vartheta, x)$ be the fundamental solution of $P \in$ hyp $(\vartheta)$ let $Q$ be a polynomial and put $F=Q(D) E, F_{\xi}=Q_{\xi}(D) E\left(P_{\xi}\right)$, $\xi$ real. Let $m(f)=m(Q)-m(P)$ be the degree of $f=Q / P$ and $m_{\xi}(f)=m_{\xi}(Q)-m_{\xi}(P)$ the multiplicity of $f$ relative to $\xi$. Then

$$
R \ni t \rightarrow \infty \Rightarrow t^{m_{\xi}(f)-m(f)} e^{-i t x \xi} F(x) \rightarrow F_{\xi}(x)
$$

in the distribution sense and

$$
\xi \neq 0 \Rightarrow \mathrm{S}\left(F_{\xi}\right) \subset \mathrm{SS}(F) .
$$

The homogeneous case. Analytic continuation. Our further analysis of fundamental solutions rests upon certain explicit formulas which show them to be holomorphic outside the wave front surface (Theorem 7.5). In order to prepare for the proofs, it is convenient to interpolate between all $E\left(a^{k}, \vartheta, x\right),(k=1,2, \ldots)$, using a complex parameter $s$. We put

$$
E_{s}(a, x)=(2 \pi)^{-n} \int a(\xi+i \eta)^{-s} e^{i x(\xi+i \eta)} d \xi, \quad \eta \in-\Gamma(A, \vartheta),
$$

10-702909 Acta mathematica. 124. Imprimé le 8 Avril 1970. 
where $s$ is a complex number. The definition of $a^{-s}$ offers no difficulties. In fact, by (3.16) and Corollary 3.18

$$
a(\xi+i \eta)=a(\eta) \prod_{1}^{m}\left(i+\lambda_{k}(\eta, \xi)\right)
$$

where $\lambda_{k}(\eta, \xi)$ is real when $\xi$ is real and $\pm \eta \in \Gamma=\Gamma(A, \vartheta)$. Hence

$$
\arg a(\xi+i \eta)=\arg a(\eta)+\sum_{1}^{m} \arg \left(i+\lambda_{k}(\eta, \xi)\right),
$$

(where $\arg i=\pi / 2$ ), is continuous and single-valued on $\operatorname{Re} Z \pm i \Gamma$ once $\arg a( \pm \vartheta)$ is fixed. The following estimates are immediate

$$
\begin{aligned}
& \operatorname{Re} s \geqslant 0 \Rightarrow\left|a(\xi+i \eta)^{-s}\right| \leqslant e^{c|s|}|a(\eta)|^{- \text {Ros }} \\
& \operatorname{Re} s \leqslant 0 \Rightarrow\left|a(\xi+i \eta)^{-s}\right| \leqslant e^{c|s|}|a(\xi+i \eta)|^{-\operatorname{Re} s}
\end{aligned}
$$

where $c$ is a constant.

4.15. Lумма. Let $a \in \operatorname{Hyp}(\vartheta)$. Then the distributions $E_{s}(x)=E_{s}(a, \vartheta, x)$ defined by (4.13) are entire analytic in s and

$$
\begin{gathered}
\lambda>0 \Rightarrow E_{s}(\lambda x)=\lambda^{m s-n} E_{s}(x) \\
\mathrm{S}\left(E_{s}\right) \subset K(A, \vartheta) \\
a(D) E_{s}(x)=E_{s-1}(x) \\
s=0,-1,-2, \ldots \Rightarrow E_{s}(x)=a(D)^{-s} \delta(x) .
\end{gathered}
$$

Note. (4.13) should be taken in the sense that

$$
\left(E_{s}, \check{g}\right)=(2 \pi)^{-n} \int a(\xi+i \eta)^{-s} \mathcal{F} g(\xi+i \eta) d \xi,
$$

where $g \in C_{0}\left(\operatorname{Re} Z^{\prime}\right)$. That the distributions (4.13) are analytic in $s$ means that all $\left(E_{s}, \check{g}\right)$ have that property.

Proof. Theorem 2.4 and simple verifications using (4.14).

Note. The idea of imbedding the fundamental solution $E=E(a, x)$ in an analytic family $E_{s}$ is due to M. Riesz (1949) who gives explicit formulas for $E_{s}$ in case $a(D)$ is the wave operator $\Delta(\partial \mid \partial x)=\left(\partial \mid \partial x_{1}\right)^{2}-\left(\partial \mid \partial x_{2}\right)^{2}-\ldots-\left(\partial \mid \partial x_{n}\right)^{2}$. The construction is also used in Gårding (1947), Schwartz (1950) and Gelfand-Shilov (1955). For later reference we state here the formulas of M. Riesz. With $a=\Delta$ as above, we have $a \in \operatorname{Hyp}(\vartheta, 2), \vartheta=(1,0, \ldots, 0)$, $\Gamma(A, \vartheta)=\left\{\xi ; \xi_{1}>0, \xi_{1}^{2}-\xi_{2}^{2}-\ldots-\xi_{n}^{2}>0\right\}, K(A, \vartheta)=\left\{x ; x_{1} \geqslant 0, x_{1}^{2}-x_{2}^{2}-\ldots \geqslant 0\right\}$ and, if $x \in$ $\stackrel{\circ}{K}(A, \vartheta)$, 


$$
E_{s}(\Delta, \vartheta, x)=\left(x_{1}^{2}-x_{2}^{2}-\ldots-x_{n}^{2}\right)^{s-\frac{1}{2} n} / \pi^{(n-2) / 2} 2^{2 s-1} \Gamma(s) \Gamma\left(s+2-\frac{1}{2} n\right) .
$$

When $\operatorname{Re} s>-1-\frac{1}{2} n$, this defines $E_{s}$ as an integrable function; when $\operatorname{Re} s \leqslant-1-\frac{1}{2} n$, we define $E_{s}$ by analytical continuation. Note in particular that if $x$ is inside the light-cone $K(A, \vartheta)$ then

$$
\begin{aligned}
2 p<n \text { even } & \Rightarrow E\left(\Delta^{p}, \vartheta, x\right)=0 \\
2 p \geqslant n \text { even } & \Rightarrow E\left(\Delta^{p}, \vartheta, x\right)=\text { const }\left(x_{1}^{2}-x_{2}^{2}-\ldots-x_{n}^{2}\right)^{p-\frac{1}{2} n} \\
n \text { odd } & \Rightarrow E\left(\Delta^{p}, \vartheta, x\right)=\text { const }\left(x_{1}^{2}-x_{2}^{2}-\ldots-x_{n}^{2}\right)^{p-\frac{1}{2} n}
\end{aligned}
$$

where $p=1,2, \ldots$.

In order to analyse the fundamental solution $E(x)=E(a, \vartheta, x)$ outside the wave front surface $W=W(A, \vartheta)$, we shall change the chain of integration in (4.13). The following is a heuristic outline of our procedure.

We are going to replace the constant vector field $\xi \rightarrow \eta$ in (4.13) by certain real smooth vector fields $\xi \rightarrow v(\xi)$ homotopic to it. By Cauchy's, or rather Stokes', theorem, this does not change the integral provided we replace $d \xi$ by $d(\xi+i v(\xi)), \xi+i v(\xi)$ stays away from the complex hypersurface $A: a(\zeta)=0$, and the exponential stays bounded, i.e. $x v(\xi)$ stays bounded from below.

We then have

$$
E_{s}(x)=(2 \pi)^{-n} \int_{\mathrm{Re} Z} a(\xi+i v(\xi))^{-s} e^{i x(\xi+i v(\xi))} d(\xi+i v(\xi)) .
$$

One way to satisfy these requirements is to choose $v(\xi)$ in $-\Gamma(A, \vartheta)$ and to keep it bounded, but there are wider possibilities. Localizing $a$ at $\xi$ we have

$$
a(\xi+i v(\xi)) \sim a_{\xi}(i v(\xi)), \quad \xi \text { large }
$$

and, keeping $v(\xi)$ bounded, it seems possible to allow

$$
v(\xi) \epsilon-\Gamma_{\xi}(A, \vartheta), \quad \xi \text { large. }
$$

As we shall see in Section 6 , all such choices of $v$ are in fact permitted. The proof uses a semi-continuity property of the cones $\Gamma_{\xi}$ proved in Section 5: the intersection of a $\Gamma_{\xi}$ with neighbouring cones is never much smaller than $\Gamma_{\xi}$ itself.

Our next step is to produce vector fields $v(\xi)$ such that the real part $-x v(\xi)$ of the exponential in (4.22) makes the integral convergent. To this end, let $X$ be the complex hyperplane $x \zeta=0$ and suppose that $0 \neq x \in \operatorname{Re} Z^{\prime}$ is such that

$$
\operatorname{Re} X \cap \Gamma_{\xi} \neq \varnothing, \quad \forall \xi \neq 0 .
$$

This condition actually characterizes the complement of $\pm W$, it means that $x \bar{\epsilon} \pm W$. We may then strengthen $(4.23)$ to 


$$
v(\xi) \epsilon-\operatorname{Re} X \cap \Gamma_{\xi}(A, \vartheta), \quad \xi \text { large }
$$

and a small perturbation $v_{\varepsilon}(\xi)$ of $v$ respecting (4.23) makes $v_{\varepsilon}(\xi) x>0$ for large $\xi$. But then we are free to let $v_{\varepsilon}(\xi)$ become large for large $\xi$, so that

$$
v_{\varepsilon}(\xi) x>c|\xi|, \quad c>0 \text {, large } \xi .
$$

With such a choice, there is absolute convergence in (4.22) also when $x$ is replaced by some near-by complex argument. This shows that $E_{s}(a, x)$ is holomorphic outside $W$.

We also want to get the explicit formulas for $E(x)=E(a, \vartheta, x)$ given in the introduction. To do this we exploit the homogeneity of $a$ by making $v_{\varepsilon}(\xi)$ positive homogeneous. Then we have convergence difficulties at the origin, but they can be avoided by restricting $s$ to the half-plane $\operatorname{Re} s<0$. Performing a radial integration and using formula (1.7) we then obtain a formula for $E_{s}(x)$ when $\operatorname{Re} s<0$ that extends by analytic continuation to $s=1$. The formula for $E(x)=E_{1}(x)$ at this step still involves the small parameter $\varepsilon$. Using the boundary values introduced in Section 1 we now let $\varepsilon \rightarrow 0$. The formula for $E(x)$ that we get unfortunately still involves a logarithm which we eliminate by the following device. Restricting $x$ to be outside $-K$, we have $E(-x)=0$. Subtracting the integral formula for $E(-x)$ from that for $E(x)$ and using (1.15) we get rid of the logarithmic term and end up, finally, with the rational integrals (4) and (5) described in the introduction. The nonhomogeneous case is taken care of by (4.5).

To carry out this programme, we have to construct vector fields satisfying (4.23) and (4.24) and study their homotopy properties. This will be done in Section 6 while Section 5 is devoted to the semi-continuity of the local cones $\Gamma_{\xi}$. The actual computations sketched above will be done in Section 7 .

\section{The geometry of hyperbolic surfaces. Semi-continuity of the local cones}

Let $a \in \operatorname{Hyp}(\vartheta)$ and $A: a(\zeta)=0$ the associated hyperbolic surface. Lemma 3.27 shows that the real singularities of a hyperbolic surface (considered in real projective space) are of a specially simple type. Any twodimensional plane through $\vartheta$ cuts the surface in an algebraic curve whose singularities are multiple crossings (not necessarily transversal) of simple branches (see figure $5 a$ ).

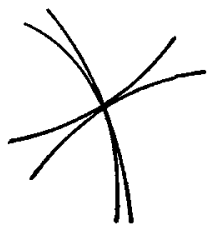

Fig. $5 a$ 
Consider the localization $a_{\xi}$ of $a$ at $\xi \in \operatorname{Re} Z$. The associated hypersurface $A_{\xi}: a_{\xi}(\zeta)=0$, in other words the tangent cone of $A$ at $\xi$, is then also hyperbolic. By the definition of $\Gamma\left(A_{\xi}, \vartheta\right)$, for any $\eta \in \Gamma_{\xi}$ and any real point $\zeta$, the equation $a_{\xi}(\zeta+t \eta)=0$ has $p$ real roots, $p=m_{\xi}(a)$ being the degree of $a_{\xi}$. Since, near the point $\xi$, the surface $A$ is approximated by its tangent cone $A_{\xi}$, it is plausible that the equation $a(\xi+\zeta+t \eta)=0$ will have $p$ small real roots for small real $\zeta$ and that this should continue to hold for every hyperbolic $b$ close to $a$. This is proved in the following lemma.

5.1. Lemma. Suppose that $a, b \in \operatorname{Hyp}(\vartheta, m), \xi \in \operatorname{Re} Z$ and let $M$ be a compact part of $\Gamma_{\xi}(A, \vartheta)$. Then

$$
\eta \in M, \zeta \in \operatorname{Re} Z, \operatorname{Im} t \neq 0 \Rightarrow b(\xi+\zeta+t \eta) \neq 0
$$

provided $t, \zeta$ are sufficiently small and $b$ is sufficiently close to a.

Proof. We normalize $b$ and $a$ so that $b(\vartheta)=a(\vartheta)=1$ and measure the distance from $b$ to $a$ by the maximal difference $|a-b|$ of corresponding coefficients. Let us factor $b$ as follows, not exhibiting the dependence on $\xi$ and $\vartheta$,

$$
b(\xi+\zeta+s \vartheta)=\prod_{1}^{m}\left(s+\mu_{k}(\zeta, b)\right)
$$

The first $p=m_{\xi}(a)$ coefficients of this polynomial,

$$
b(\xi+\zeta+s \vartheta)=\sum_{0}^{p-1} f_{k}(\zeta, b) s^{k}+f_{p}(\zeta, b) s^{p}+\ldots,
$$

vanish when $b=a$ and $\zeta=0$ while $f_{p}(0, a)=a_{\xi}(\vartheta) \neq 0$. It follows that if $|\zeta|$ and $|b-a|$ are sufficiently small, then $p$ of the numbers $\mu_{k}$ in (5.3) are small while the others are bounded away from the origin. In the sequel let $|\zeta|$ and $|b-a|$ be small and let $-\mu_{1}, \ldots,-\mu_{p}$ be the small zeros of (5.3). We shall relate $\mu_{1}, \ldots, \mu_{p}$ to the numbers $\mu_{1}^{0}, \ldots, \mu_{p}^{0}$ defined by factorizing $a_{\xi}$,

$$
a_{\xi}(\zeta+s \vartheta)=a_{\xi}(\vartheta) \prod_{1}^{p}\left(s+\mu_{k}^{0}(\zeta)\right)
$$

In the first place, since the zeros of a polynomial are (multivalued) continuous functions of the coefficients, with a suitable labelling we have

$$
\mu_{k}(\zeta, b)-\mu_{k}(\zeta, a)=o_{b}(1), \quad 1 \leqslant k \leqslant m,
$$

where $o_{b}(1)$ tends to zero as $|b-a| \rightarrow 0$, uniformly for small $\zeta$. For the same reason, since

$$
\tau^{-p} a(\xi+\tau \xi+\tau s \vartheta)=\prod_{1}^{p}\left(s+\tau^{-1} \mu_{k}(\tau \zeta, a)\right) \prod_{p+1}^{m}\left(\tau s+\mu_{k}(\tau \zeta, a)\right)
$$


and also

$$
\tau^{-p} a(\xi+\tau \zeta+\tau s \vartheta)=a_{\xi}(\zeta+s \vartheta)+O(\tau)
$$

it follows that, with a suitable labelling,

$$
\tau \rightarrow 0,1 \leqslant k \leqslant p \Rightarrow \tau^{-1} \mu_{k}(\tau \zeta, a) \rightarrow \mu_{k}^{0}(\zeta)
$$

uniformly when $|\zeta|=1$. Hence, since the $\mu_{k}^{0}(\zeta)$ are homogeneous of degree 1,

$$
\mu_{k}(\zeta, a)=\mu_{k}^{0}(\zeta)+o(|\zeta|)
$$

Combinining this with (5.4) we get

$$
\mu_{k}(\zeta, b)=\mu_{k}^{0}(\zeta)+o(|\zeta|)+o_{b}(1)
$$

Now let $\eta \in M$ and replace $\zeta$ in (5.3) by $\zeta+\eta \eta$ and put $s=0$ there. The result is

$$
b(\xi+\zeta+t \eta)=\prod_{1}^{p} \mu_{k}(\zeta+t \eta, b) \prod_{p+1}^{m} \mu_{j}(\zeta+t \eta, b) .
$$

Since $b \in$ Hyp $(\vartheta)$ and $\zeta, \eta$ are real, by Lemma 3.27 we can arrange the labelling so that the functions

$$
t \rightarrow \mu_{k}(\zeta+t \eta, b), \quad 1 \leqslant k \leqslant p
$$

are differentiable for small $t$. On the other hand, it is clear that

$$
\mu_{k}^{0}(\zeta+t \eta)=t \mu_{k}^{0}(\eta)+o_{\zeta}(1), \quad 1 \leqslant k \leqslant p,
$$

where $o_{\xi}(1) \rightarrow 0$ as $\zeta \rightarrow 0$, uniformly when $\eta \in M$ and $t$ is small. Combining this with (5.5) we have

$$
\mu_{k}(\zeta+t \eta, b)=t\left(\mu_{k}^{0}(\eta)+o_{t}(1)\right)+o_{\zeta, b}(\mathbf{1}) \quad \mathbf{1} \leqslant k \leqslant p,
$$

where $o_{t}(1) \rightarrow 0$ as $t \rightarrow 0$, uniformly for small $\zeta,|b-a|$ and for $\eta \in M$ and $o_{\zeta, b}(1) \rightarrow 0$ as $\zeta$, $|b-a| \rightarrow 0$, uniformly for small $t$ and for $\eta \in M$. Now the numbers $\mu_{k}^{0}(\eta)$ have a positive lower bound on $M$ and hence (5.8) shows that to every sufficiently small $\varepsilon>0$ there is a $\delta=\delta(\varepsilon)>0$ such that every function (5.6) has at least one real zero $t=t(\zeta, \eta, b)$ with $|t|<\varepsilon$ when $\eta \in M,|\zeta|<\delta,|a-b|<\delta$. Hence, under the same conditions, the equation $b(\xi+\zeta+t \eta)=0$ has at least $p$ real roots $t$ with $|t|<\varepsilon$. But we know that the number of small roots is precisely $p$. Hence the lemma follows. We can now prove that the cones $\Gamma_{\xi}(A)$, considered as functions of $\xi$ and $a$ are semi-continuous in the sense explained below.

5.9. LеммA. Let $\xi, \zeta \in \operatorname{Re} Z$ and $a, b \in \operatorname{Hyp}(\vartheta, m)$. Then $\Gamma_{\xi+\zeta}(B, \vartheta)$ contains any preassigned compact subset $M$ of $\Gamma_{\xi}(A, \vartheta)$ provided $\zeta$ is sufficiently small and $b$ is sufficiently close to $a$. 
Proof. Let $q=m_{\xi+\xi}(b)$ be the multiplicity of $\xi+\zeta$ relative to $b$, so that

$$
b(\xi+\zeta+s \vartheta)=s^{q}\left(b_{\xi+\zeta}(\vartheta)+O(s)\right),
$$

where, the coefficient of $s^{q}$ does not vanish (since by Lemma 3.42, $b_{\xi+\xi} \in$ Hyp $(\vartheta)$ ). Taking $M$ convex and containing $\eta$, it suffices to show that $b(\xi+\zeta+t \eta)$ vanishes precisely of order $q$ at $t=0$ when $\eta \in M, \zeta$ is sufficiently small and $b$ is sufficiently close to $a$. In fact, then $b_{\xi+\xi}(\cdot)$ does not vanish on $M$ so that $\Gamma_{\xi+\zeta}(B, \vartheta) \supset M$. We shall prove this by a slight modification of the arguments of Lemma 3.42. By (5.3)

$$
b(\xi+\zeta+t \eta+s \vartheta)=\prod_{1}^{p}\left(s+\mu_{k}(\zeta+t \eta, b)\right) \prod_{p+1}^{m}(s+\ldots)
$$

and, by the preceding lemma and the convexity of $\Gamma_{\xi}(A, \vartheta)$,

$$
\operatorname{Im} s \geqslant 0, \quad \operatorname{Im} t \geqslant 0, \quad \operatorname{Im}(s+t)>0 \Rightarrow b(\xi+\zeta+t \eta+s \vartheta) \neq 0
$$

when $\zeta$ is real, $\eta$ belongs to $M, b$ is sufficiently close to $a$ and $\zeta, s, t$ are sufficiently small. Hence, under the same conditions,

$$
\operatorname{Im} t>0 \Rightarrow \operatorname{Im} \mu_{k}(\zeta+t \eta, b)>0, \quad 1 \leqslant k \leqslant p .
$$

As in the proof of Lemma 3.42, this shows that the functions $\mu_{k}$ can be labelled so that

$$
t \rightarrow \mu_{k}(\zeta+t \eta, b)
$$

are differentiable functions of $t$ with positive derivatives. Hence

$$
\mu_{k}(\zeta+t \eta, b)=\mu_{k}(\zeta, b)+c_{k} t(1+O(t))
$$

with all $c_{k}>0$. Hence, using (5.10), we see that $b(\xi+\zeta+s \vartheta)$ and $b(\xi+\zeta+t \eta)$ vanish to the same order when $s=0$ and $t=0$ respectively. But this order is $q$ and the proof is finished.

Before proceeding further, it is convenient to introduce the concepts of inner and outer continuity of functions $\tau \rightarrow C_{\tau}$ from some topological space to conical sets $C_{\tau}$ in $R^{n}$. We say that such a function is inner continuous if given any $\tau_{0}$ and a closed conical subset $N$ of $C_{\tau_{0}} \cup\{0\}$, then $C_{\tau} \supset \dot{N}=N-\{0\}$, if $\tau$ is close enough to $\tau_{0}$. By the preceding lemma, the function $a, \xi \rightarrow \Gamma_{\xi}(A, \vartheta)$ is inner continuous when $a \in \operatorname{Hyp}(\vartheta, m), \xi \in \operatorname{Re} Z$. Outer continuity is defined analogously: given any $\tau_{0}$ and any open conical set $N$ containing $\dot{C}_{\tau_{0}}$, then $\dot{C}_{\tau} \subset N$ when $\tau$ is close enough to $\tau_{0}$. Here $\dot{C}=C-\{0\}$. We now have

5.11. CoR OLL ARY. The local propagation cones $K_{\xi}(A, \vartheta)$ are outer continuous functions of $\xi \in \operatorname{Re} Z, a \in \operatorname{Hyp}(\vartheta, m)$. 
Proof. If $C$ is a closed convex proper cone and $T$ is an open conical neighbourhood of $\dot{C}$, then its dual $T^{\prime}=\left\{x ; x \in \operatorname{Re} Z^{\prime}, x T \geqslant 0\right\}$ is a closed subset of $\dot{B} \cup\{0\}$ where $B$ is the dual of $C$.

Conical algebraic hypersurfaces and their duals. To provide some background for the wave front surface we shall first deal with some generalities about conical surfaces.

When $a \neq 0$ is a homogeneous polynomial, let $A$ be the complex hypersurface $a(\zeta)=0$. Localizing $a$ at $\xi$ we get a new hypersurface $A_{\xi}: a_{\xi}(\zeta)=0$, the tangent cone of $A$ at $\xi$. The corresponding lineality $L_{\xi}=L_{\xi}(A)=L\left(A_{\xi}\right)$ is called the tangent edge of $A$ at $\xi$. Let $m$ be the degree of $a$ and let $p=m_{\xi}(a)$. Then

$$
a(\xi+s \zeta)=s^{p} a_{\xi}(\zeta)+O\left(s^{p+1}\right)
$$

so that, by the homogeneity of $a, a_{\lambda \xi}=\lambda^{m-p} a_{\xi}$ when $\lambda \neq 0$. Hence $A_{\xi}$ only depends on the ray $\dot{C} \xi$. It is obvious that $L_{\xi} \supset L=L(A)$ with equality when $\xi \in L$ so that $a_{\xi}=a$. Moreover, $\xi \in L_{\xi}$ for all $\xi$. In fact, this is the last statement of Lemma 3.52 and its proof only uses the homogeneity of $a$.

Examples. When $a(\xi) \neq 0, A_{\xi}$ is empty and $L_{\xi}=Z$. When $a(\xi)=0$, $\operatorname{grad} a(\xi) \neq 0$, then $A_{\xi}=L_{\xi}$ is the hyperplane $\operatorname{grad} a(\xi) \zeta=0$. When $a(\xi)=0$ and, locally at $\xi$,

$$
a(\zeta)=f_{1}(\zeta)^{p_{1}} \ldots f_{r}(\zeta)^{p_{r}}
$$

is a product of holomorphic factors vanishing at $\xi$ but with non-vanishing gradients there, then $A_{\xi}$ is the union of the hyperplanes $\operatorname{grad} f_{q}(\xi) \zeta=0$ while $L_{\xi}$ is their intersection.

As usual in agebraic geometry, a point $\xi \in A$ is said to be regular if, close to $\xi, A$ has an equation $f(\zeta)=0$ where $f$ is holomorphic and $\operatorname{grad} f(\xi) \neq 0$. Any part of $A$ consisting of regular points is said to be regular.

The real part of $A$, defined as the set of real $\xi$ such that $a(\xi)=0$ will be denoted by $\operatorname{Re} A$. A point $\xi \in \operatorname{Re} A$ is said to be regular if it is regular in $A$ and parts of $\operatorname{Re} A$ consisting of regular points are said to be regular.

An $x \in Z^{\prime}$ is said to be normal to $A$ at $\xi$ if $x L_{\xi}(A)=0$. The corresponding hyperplane $X: x \zeta=0$, is then tangent to $A$ at $\xi$, i.e. $X \supset L_{\xi}(A)$. All $x$ normal to $A$ at $\xi$ constitute the normal of $A$ at $\xi$, i.e. the orthogonal complement $L_{\xi}^{0}(A)$ in $Z^{\prime}$ of the lineality $L_{\xi}(A)$. The normal at $\xi=0$ is the orthogonal complement $L^{0}(A)$ of $L(A)$.

Examples. When $a(\xi) \neq 0$, the normal at $\xi$ vanishes. When $a(\xi)=0$ but $\operatorname{grad} a(\xi) \neq 0$, the normal at $\xi$ is spanned by grad $a(\xi)$. When $a$ has the factorization (5.12) at $\xi$, the normal at $\xi$ is simply the linear span of all $\operatorname{grad} f_{Q}(\xi)$. When $\xi \in A$ is a regular point, the normal 
at $\xi$ is a complex line, but this may also happen at singular points, e.g. if in (5.12) all $\operatorname{grad} f_{a}(\xi)$ are proportional.

All normals of $A$ at points $\xi \neq 0$ constitute the dual surface ${ }^{\circ} A$ of $A$. Note that ${ }^{0} A \subset L^{0}(A)$. In fact, $L_{\xi}(A) \supset L(A)$ for all $\xi$. Since $L_{\xi}(A)=L(A)$ when $\xi \in L(A),{ }^{0} A$ coincides with $L^{0}(A)$ if $L(A) \neq 0$. In general, ${ }^{\circ} A$ is a rather complicated object which we shall not analyse much further except for proving

Proposition. The dual ${ }^{0} A$ of $A$ is contained in a proper conical subvariety.

Note. With a slightly modified definition of ${ }^{0} A$, viz. as the union of normals $L_{\xi}^{0}(A)$ for $\xi \bar{\epsilon} L(A),{ }^{0} A$ is contained in a proper conical subvariety of $L^{0}(A)$. This follows from the proposition by a passage from $Z$ to the quotient $Z / L(A)$.

Proof. Let $A_{k} \subset A$ be the set of points of multiplicity $\geqslant k$. Thus

$$
\xi \in A_{k} \Leftrightarrow(\partial / \partial \xi)^{\alpha} a(\xi)=0 \text { for }|\alpha|<k,
$$

showing that $A_{k}$ is a closed subvariety of $Z$. Hence $B_{k}=A_{k}-A_{k+1}$ is a Zariski open set on $A_{k}$ and so is a locally closed subvariety of $Z$. For $\xi \in B_{k}$, the local lineality $L_{\xi}(A)$ is the space of all $\eta$ such that

$$
\left(\sum \eta_{i} \partial / \partial \zeta_{i}\right) \sum_{|\alpha|=k}(\alpha !)^{-1}(\partial / \partial \xi)^{\alpha} a(\xi) \zeta^{\alpha} \equiv 0 \text { in } \zeta
$$

This shows that the subset $B_{k, s}$ of all $\xi \in B_{k}$ such that $n_{\xi}(a)=s$ is a locally closed algebraic subvariety and the spaces $L_{\xi}(A)$ then form an algebraic vector bundle of fibre dimension $n-s$ over $B_{k, s}$ and also over the image $B_{k, s}^{*}$ of $\dot{B}_{k, s}$ in projective space $Z^{*}=\dot{Z} / \dot{C}$. The spaces $L_{\xi}^{0}(A)$ will then form an algebraic vector bundle over $B_{k, s}^{*}$ of fibre dimension $s$. The Zariski closure of the set

$$
C_{k, s}=\bigcup L_{\xi}^{0}(A), \quad \xi \in B_{k, s}^{*}
$$

is therefore an algebraic subvariety of $Z^{\prime}$ of dimension $\leqslant s+\operatorname{dim} B_{k, s}^{*}$. Now, from the definition of ${ }^{0} \mathrm{~A}$ we easily see that

$$
{ }^{0} A=\mathrm{U} C_{k, s}, \quad \mathrm{l} \leqslant k \leqslant m, \quad \mathrm{l} \leqslant s \leqslant n-\mathrm{I} .
$$

In fact, when $L_{\xi}^{0}(A) \neq 0$, i.e. $L_{\xi}(A) \neq Z$, and $\xi \neq 0$ then $a(\xi)=0$ so that $1 \leqslant m_{\xi}(a) \leqslant m$ and, since $\xi \in L_{\xi}(A), 1 \leqslant n_{\xi}(a) \leqslant n-1$. Hence, to prove the proposition it only remains to show that, for all values of $k, s$ being considered,

$$
s+\operatorname{dim} B_{k, s}^{*} \leqslant n-1 \text {. }
$$

Suppose therefore that $d=d(k, s)=\operatorname{dim} B_{k, s}$ and let $\xi$ be a non-singular point of $B_{k, s}$. 
Then we can choose local analytic coordinates $(u, v)$ for $Z$ centered at $\xi$ so that $B_{k, s}$ is given locally by the equations $v=0$; here $u=\left(u_{1}, \ldots, u_{d}\right)$ and $v=\left(v_{1}, \ldots, v_{n-d}\right)$. Let $f(u, v)$ be the analytic function which represents the polynomial $a$ in these coordinates. Then the multiplicity of $f$ at any point $\zeta$ in the domain of these coordinates is equal to $m_{\zeta}(a)$ and hence, for all $\zeta \in B_{k, s}$, it is equal to $k$. In particular, when $u$ is small and fixed, $f$ vanishes of order $k$ at $v=0$. This shows that $f$ has an expansion around $u=v=0$ of the form $f=f_{k}+f_{k+1}+\ldots$ where $f$ is homogeneous of degree $j$ and

$$
f_{k}=\sum c_{\alpha} v^{\alpha}, \quad|\alpha|=k
$$

is independent of $u$. Now $f_{k}$ corresponds to the homogeneous polynomial $a_{\xi}$ except for a linear change of variables. Hence $a_{\xi}$ depends on $\leqslant n-d$ variables. In other words $s=n_{\xi} \leqslant n-d$ and so

as required.

$$
s+\operatorname{dim} B_{k, s}^{*}=s+d-1 \leqslant n-1
$$

When $a$ is a real polynomial apart from a possible complex constant factor, and $\xi$ is real, the tangent edge $L_{\xi}(A)$ and the normal $L_{\xi}^{0}(A)$ are the complexifications of their real parts $\operatorname{Re} L_{\xi}(A)$ and $\operatorname{Re} L_{\xi}^{0}(A)$. We shall be interested in the real dual ${ }^{\circ} \operatorname{Re} A$ of $\operatorname{Re} A$ defined as the union of the real normals $\operatorname{Re} L_{\xi}^{0}(A)$ for $\xi$ real and $\neq 0$. It is clear that ${ }^{0} \operatorname{Re} A \subset \operatorname{Re}{ }^{0} A$ and hence, by the previous proposition, ${ }^{0} \mathrm{Re} A$ is contained in a proper algebraic subvariety of $Z^{\prime}$. In other words, ${ }^{0} \operatorname{Re} A$ has codimension $\geqslant 1$ everywhere.

The wave front surface. When $a \in \mathrm{Hyp}(\vartheta)$ and $\xi$ is real, we know from Lemma 3.42 that $a_{\xi} \in \mathrm{Hyp}(\vartheta)$ and we have defined the local cones $\Gamma_{\xi}=\Gamma_{\xi}(A, \vartheta)=\Gamma\left(A_{\xi}, \vartheta\right)$ and their duals $K_{\xi}=K_{\xi}(A, \vartheta)=K\left(A_{\xi}, \vartheta\right)$. We know that

$$
\Gamma_{\xi} \supset \Gamma=\Gamma(A, \vartheta), \quad K_{\xi} \subset K=K(A, \vartheta)
$$

when $\xi \in \operatorname{Re} L(A)$, there is equality. Also, by Lemma 3.52

so that

$$
x \in K_{\xi} \Rightarrow \operatorname{Re} X \supset \operatorname{Re} L_{\xi}
$$

$$
K_{\xi}(A, \vartheta) \subset{ }^{0} \operatorname{Re} A, \quad \vee \text { real } \xi .
$$

Examples. When $a(\xi) \neq 0$, then $\Gamma_{\xi}=\operatorname{Re} L_{\xi}=\operatorname{Re} Z, K_{\xi}=\{0\}$. When $a(\xi)=0$, but grad $a(\xi) \neq 0$, then $\Gamma_{\xi}$ is the half-space $(\operatorname{grad} a(\xi) \cdot \vartheta)^{-1}(\operatorname{grad} a(\xi) \cdot \zeta)>0$ while $K_{\xi}$ is the nonnegative span of $(\operatorname{grad} a(\xi) \cdot \vartheta)^{-1} \operatorname{grad} a(\xi)$, i.e. one-half of the real normal. If $a$ is strongly hyperbolic or, more generally, $\operatorname{Re} A$ has at most one-dimensional normals, these exhaust all possibilities. When $a(\zeta) / a(\vartheta)$ has the factorization (5.12) at $\xi$ with real and homogeneous 
factors, then $\Gamma_{\xi}$ is the intersection of the half-spaces $\left(\operatorname{grad} f_{s}(\xi) \cdot \vartheta\right)^{-1}\left(\operatorname{grad} f_{s}(\xi) \cdot \zeta\right)>0$ while $K_{\xi}$ is the non-negative linear span of the corresponding real normals ( $\left.\operatorname{grad} f_{s}(\xi) \cdot \vartheta\right)^{-1}$ $\operatorname{grad} f_{s}(\xi)$.

We now come to the wave front surface.

5.15. Definition. When $a \in \operatorname{Hyp}(\vartheta)$, let

$$
W(A, \vartheta)=\bigcup K_{\xi}(A, \vartheta), \quad \xi \in \operatorname{Re} \dot{Z}
$$

be the wave front surface.

It follows from (5.13) that $W(A, \vartheta) \subset K(A, \vartheta)$ with equality if $L(A) \neq 0$, i.e. if the polynomial $a$ is not complete. In view of the definition of $K_{\xi}(A, \vartheta), x \in W(A, \vartheta)$ if and only if $x \Gamma_{\xi}(A, \vartheta) \geqslant 0$ for at least one $\xi \in \operatorname{Re} \dot{Z}$. Hence

$$
x \bar{\epsilon} \pm W(A, \vartheta) \Leftrightarrow \Gamma_{\xi}(A, \vartheta) \cap \operatorname{Re} X \neq \varnothing, \quad \forall \xi \in \operatorname{Re} \dot{Z},
$$

which is a useful characterization of the complement of $\pm W(A, \vartheta)$.

5.17. Lемма. Let $a \in \operatorname{Hyp}(\vartheta, m)$.

a) The wave front surface $W=W(A, \vartheta)$ is a closed subset of $K=K(A, \vartheta)$ and

$$
\partial K \subset W \subset K \cap{ }^{0} \operatorname{Re} A
$$

with equality on the right when $\operatorname{Re} A$ has as most one-dimensional normals or when $L(A) \neq 0$ which case $W=K \subset{ }^{0} \operatorname{Re} A$.

b) The function $a \rightarrow W(A, \vartheta)$ is outer continuous. There are operators $Q$ in $\operatorname{hyp}^{0}(\vartheta, m)$ which are arbitrarily close to a given $P \in \mathrm{hyp}(\vartheta, m)$ and such that $W(Q, \vartheta)$ meets any given conical neighbourhood of a ray in $W(P, \vartheta)$.

Proof. a) That $W \subset K$ follows from (5.13). To prove that $W$ is closed, note that if $x \bar{\epsilon} K_{\xi}=K_{\xi}(A, \vartheta)$ for some $x$ and $\xi \in \operatorname{Re} \dot{Z}$, then by the outer continuity of $K_{\xi}$ as a function of $\xi, y \bar{\epsilon} K_{\eta}$ when $y, \eta$ are real and sufficiently close to $x$ and $\xi$ respectively. Hence, since the manifold $|\xi|=1$ is compact, $y \bar{\epsilon} K_{\eta}$ for all real $\eta \neq 0$ when $y$ is sufficiently close to $x$. Hence $W$ is closed. In this line of reasoning, using also the outer continuity of $K_{\xi}(A, \vartheta)$ with respect to $a$, we conclude that every $x$ outside $W=W(A, \vartheta)$ has an open conical neighbourhood which is outside $W(B, \vartheta)$ when $b \in \mathrm{Hyp}(\vartheta, m)$ is sufficiently close to $a$. Hence, if $T$ is an open conical neighbourhood of $\dot{W}$, covering $\operatorname{Re} \dot{Z}-T$ by a finite number of open cones, we conclude that $\dot{W}(B, \vartheta) \subset T$ when $b$ is close enough to $a$. Hence $W(A, \vartheta)$ is an outer continuous function of $a$. 
The right inclusion (5.18) follows from (5.14). To prove the left inclusion, let $0 \neq x \in \partial K$. Then $x \Gamma>0, \Gamma=\Gamma(A, \vartheta)$ and there exists a $0 \neq \xi \in \partial \bar{\Gamma}$ such that $x \xi=0$. Let $\eta \in \Gamma_{\xi}$. Then, since $\Gamma_{\xi}$ is convex and contains $\vartheta, s \vartheta+(1-s) \eta \in \Gamma_{\xi}$ when $0 \leqslant s \leqslant 1$. Since

$$
a(\xi+t(s \vartheta+(1-s) \eta))=t^{p}\left(a_{\xi}(s \vartheta+(1-s) \eta)+O(t)\right)
$$

where $p=m_{\xi}(a)$, it follows that there exists a $t_{0}>0$ such that $a\left(\xi+t_{0}(s \vartheta+(1-s) \eta)\right) \neq 0$ when $0 \leqslant s \leqslant 1$. Since $\xi+t_{0} \vartheta \in \Gamma$ we conclude therefore that $\xi_{0}+t_{0} \eta \in \Gamma$ so that $x\left(\xi+t_{0} \eta\right)=$ $t_{0} x \eta>0$ and, consequently, $x \in K_{\xi}$. Hence $\partial K \subset W$. Since $a_{\xi}=a$ when $\xi \in L=L(A)$, we have $W=K \subset{ }^{0} \operatorname{Re} A$ when $L \neq 0$. When $L=0$, then ${ }^{0} \operatorname{Re} A$ has codimension $\geqslant 1$ in $\operatorname{Re} Z$ and hence $W$ has the same property. When $\operatorname{Re} A$ is regular, then every $0 \neq \xi \in \operatorname{Re} A$ has precisely one real normal, half of it being $K_{\xi}$. Hence $W=K \cap{ }^{0} \operatorname{Re} A$ in that case. As shown by the examples below, $W$ may be smaller than $K \cap{ }^{\circ} \operatorname{Re} A$ when $\operatorname{Re} \cdot A$ has normals of dimension $>1$.

b) We know already that $W(a, \vartheta)$ is an outer continuous function of $a$. Let $f(t)$ be a polynomial in one variable whose zeros lie in the band $|\operatorname{Im} t|<c$. Then, if $s$ is real, the zeros of $f(t)-s f^{\prime}(t)$ lie in the same band. The proof of this remark is left to the reader. Let $a$ be the principal part of $P \in$ hyp $(\vartheta, m)$ and let $c(\xi)$ be a real linear form with $c(\vartheta)=0$ and put

$$
Q(\xi)=\left(1-\varepsilon c(\xi) \nabla_{\vartheta}\right) P(\xi)
$$

where $\nabla_{\vartheta}$ is differentiation along $\vartheta$. The principal part of $Q$ is

$$
b(\xi)=\left(1-\varepsilon c(\xi) \nabla_{\vartheta}\right) a(\xi)
$$

we have $b(\vartheta)=a(\vartheta)$ and, by the remark above, $Q \in$ hyp $(\vartheta, m)$. In the sequel, $P$ is supposed to be normalized so that $a(\vartheta)>0$. Let $P_{\xi}$ with principal part $a_{\xi}$ be the localization of $P$ at some point $\xi \in \operatorname{Re} A$, let $\eta \in \Gamma_{\xi}=\Gamma_{\xi}(A, \vartheta)$ and consider $Q(\xi+s \eta)$. Let $p=m_{\xi}(a)>0$ be the multiplicity of $\xi$. A short calculation gives

$$
b(\xi+s \eta)=s^{p-1}\left(\left(a_{\xi}(\eta)-\varepsilon c(\eta) \nabla_{\vartheta} a_{\xi}(\eta)\right) s-\varepsilon c(\xi) \nabla_{\vartheta} a_{\xi}(\eta)+O\left(s^{2}\right)\right)
$$

where $\nabla_{\vartheta}$ operates on $\eta$. Hence the polynomial $s \rightarrow b(\xi+s \eta)$ has a $(p-1)$-fold zero at the origin and one small zero

$$
s=s_{0}=\varepsilon c(\xi) a_{\xi}(\eta)^{-1} \nabla_{\vartheta} a_{\xi}(\eta)+O\left(\varepsilon^{2}\right)
$$

Here, since $\eta \in \Gamma_{\xi}, a_{\xi}(\eta)$ is positive and by Lemma 3.56, $\nabla_{\vartheta} a_{\xi}(\eta)$ is also positive. Calculating the gradient of $b$ gives 


$$
(\nabla b)(\xi+s \eta)=s^{p-2}\left(\nabla a_{\xi}(\eta) s-\varepsilon c(\xi) \nabla \nabla_{\vartheta} a_{\xi}(\eta)+O\left(\varepsilon^{2}\right)\right)
$$

where $\nabla$ operates on $\eta$ on the right. Now choose $c$ such that $c(\xi)>0$ and let $\varepsilon>0$ be small. Then the zero (5.19) is positive for small $\varepsilon$ and inserting its value in $b$ gives

$$
(\nabla b)\left(\xi+s_{0} \eta\right)=c_{0} \varepsilon^{p-1}\left(a_{\xi}(\eta)^{-1} \nabla_{\vartheta} a_{\xi}(\eta) \nabla a_{\xi}(\eta)-\nabla \nabla_{\vartheta} a_{\xi}(\eta)+O(\varepsilon)\right)
$$

where $c_{0}$ is a positive number independent of $\varepsilon$. Rewriting the right side we have

$$
(\nabla b)\left(\xi+s_{0} \eta\right)=c_{0} \varepsilon^{p-1} a_{\xi}(\eta)\left(\nabla \nabla_{\vartheta} \log a_{\xi}(\eta)^{-1}+O(\varepsilon)\right)
$$

Now let $R(\eta)=R_{\xi}(\eta)$ be the positive ray spanned by the first term of the parenthesis. By virtue of Lemma 3.56 , these rays constitute the interior of $K_{\xi}=K_{\xi}(A, \vartheta)$ relative to $L_{\xi}^{0}(A)$, Hence, as $\varepsilon \rightarrow 0$, the positive rays spanned by the left side of $(5.20)$ with $\eta \in \Gamma_{\xi}$ come as close as we want to any ray in this interior. This means in particular that, as $\varepsilon \rightarrow 0$, $W(B, \vartheta)$ comes as close as we want to any ray in $K_{\xi}$. Now $B$ may not be strongly hyperbolic but repeating the operation $P \rightarrow Q$ used above a number of times with different $\varepsilon$ and linear functions $c(\xi)$ but now starting from $Q$ we can construct a $Q^{\prime} \in \operatorname{hyp}^{0}(\vartheta, m)$ whose coefficients tend to those of $Q=Q_{\varepsilon}$ with fixed $\varepsilon>0$. (This is the procedure used by Nuij (1969).) But then, if $\xi, \eta \in \Gamma_{\xi}$ and $\varepsilon>0$ are fixed and $b^{\prime}$ denotes the principal part of $Q^{\prime}$, it is clear that $\nabla b^{\prime}\left(\xi+s_{0} \eta\right)$ tends to $\nabla b\left(\xi+s_{0} \eta\right)$ as $b^{\prime}$ tends to $b$ and this finishes the proof.

Examples. The following figures show the images in real projective two-space of some $\operatorname{Re} A$ and $W(A, \vartheta)$ when $n=3$ and $a \in \operatorname{Hyp}(\vartheta, m), m=2,3,4,6$. The polynomial $a$ is supposed to be strongly hyperbolic except in the cases $3,5,6,7,8,9$ and in the cases $3,5,6,8,9$, the dotted lines indicate $\operatorname{Re} B$ and $W(B, \vartheta)$ for a strongly hyperbolic $b \in \operatorname{Hyp}(\vartheta)$ close to $a$. In all examples, $K(A, \vartheta)$ is bounded by the outer contours to the right. The general properties of $\operatorname{Re} A$ and ${ }^{\circ} \operatorname{Re} A$ considered as algebraic curves are well known. In particular, a point of inflexion and a double point of $\operatorname{Re} A$ correspond to a cusp and a double tangent respectively in its dual ${ }^{\circ} \operatorname{Re} A$. In all cases we get the dual ${ }^{\circ} \operatorname{Re} A$ by adding to $W(A, \vartheta)$ all its multiple tangents. Segments of double tangents appear in $W(A, \vartheta)$ in the examples $3,5,7,8,9$. In example 5, $W(A, \vartheta)$ has a double tangent intersecting $W(A, \vartheta)$ in a point. The examples $3,5,6$ illustrate the fact that while $W(A, \vartheta)$ is an outer continuous function of $a,{ }^{0} \operatorname{Re} A$ does not have that property.

The examples 6 and 9 occur in two-dimensional crystal optics and magnetogasdynam. ics respectively (see Courant-Hilbert 1962, II. 599-617). The example 10 is copied from Borovikov (1961). Here Re $A$ is close to three intersecting ellipses. 


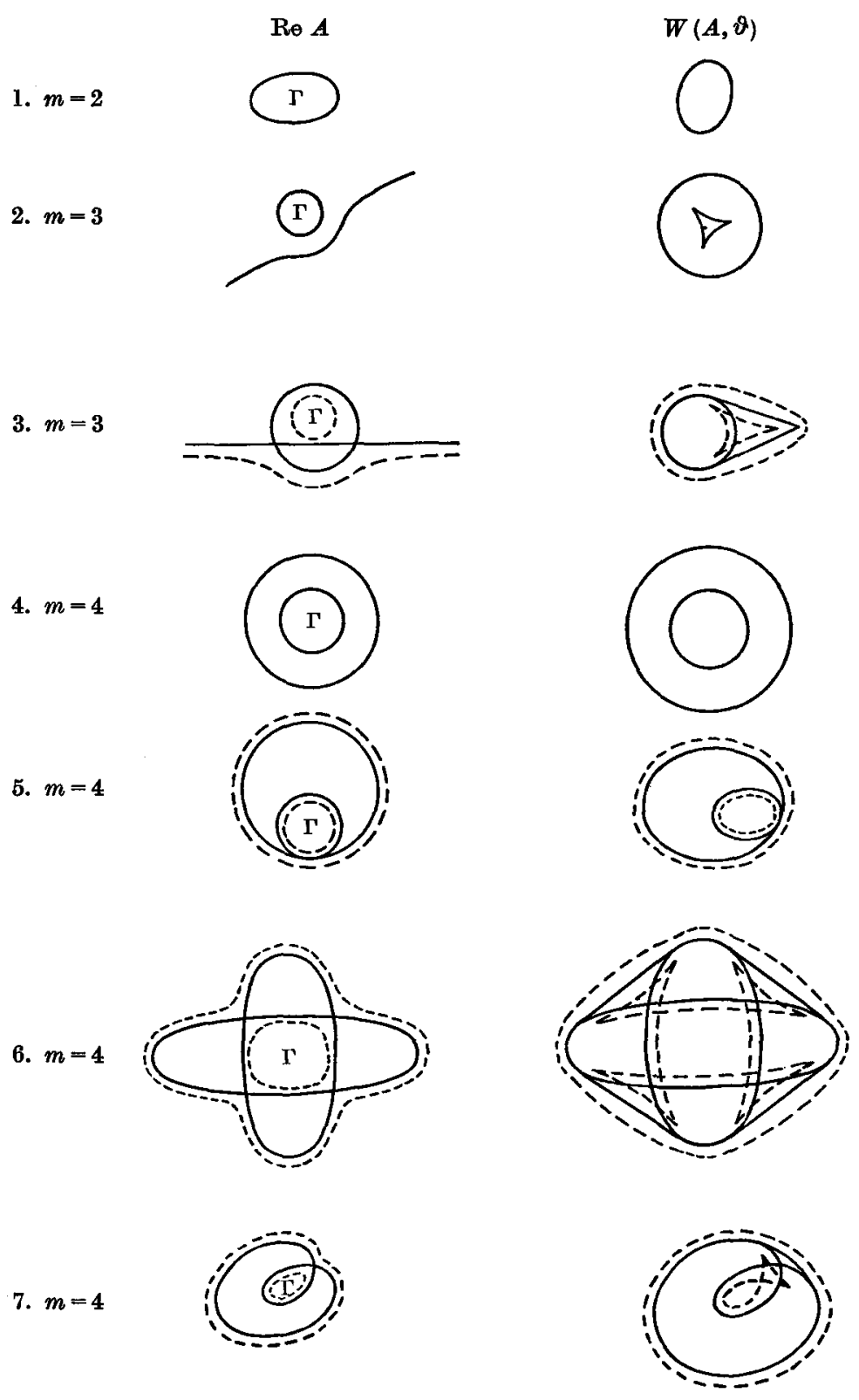

Fig. $5 b$ 

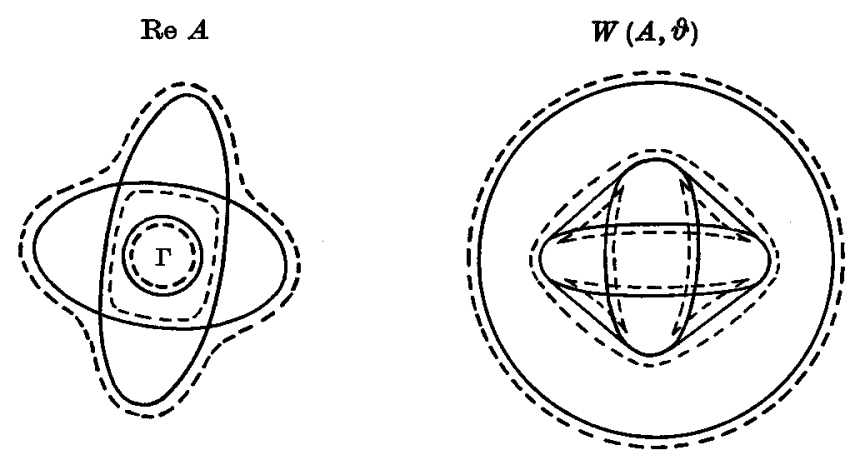

8. $m=6$
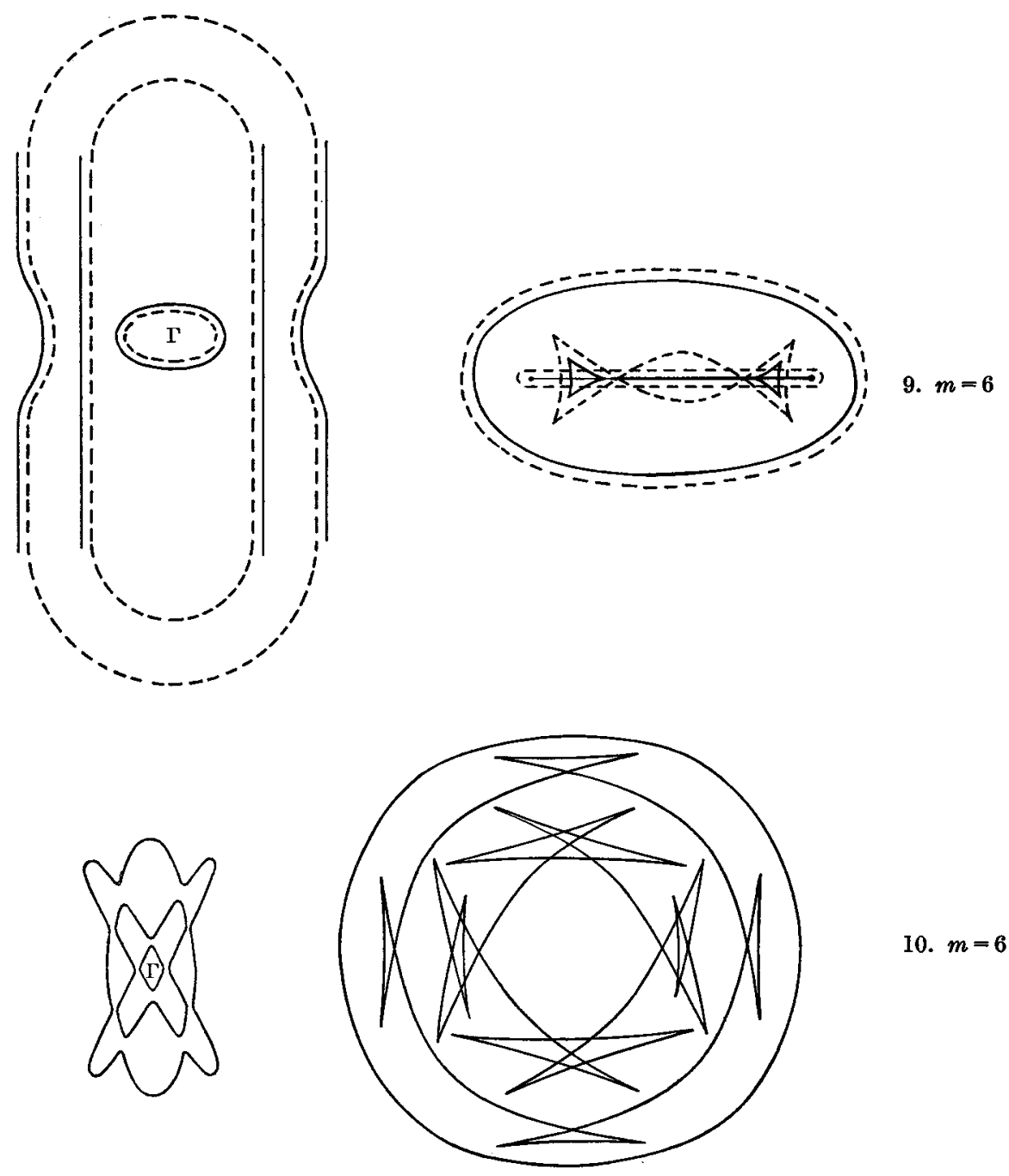

10. $m=6$

Fig. $5 b$ 


\section{Vector fields and cycles}

Let $a \in \operatorname{Hyp}(\vartheta)$. Following the heuristic outline at the end of Section 4, we shall now construct certain maps from $\operatorname{Re} \dot{Z}$ to $Z-A$ homotopic to the maps $\xi \rightarrow \xi+i \eta, \eta \in \Gamma(A, \vartheta)$. We shall only consider $C^{\infty}$ vector fields $\operatorname{Re} \dot{Z} \ni \xi \rightarrow v(\xi) \in \operatorname{Re} Z$ which are absolutely homogeneous

$$
\lambda \in \dot{R} \Rightarrow v(\lambda \xi)=|\lambda| v(\xi)
$$

Let $U$ be the family of such fields topologized by uniform convergence of $v$ and all its derivatives when $|\xi|=1$. It is convenient to define certain subfamilies of $U$.

6.2. Definition. Let $U(A, \vartheta)$ be all $v \in U$ such that

$$
v(\xi) \in \Gamma_{\xi}(A, \vartheta), \quad \forall \xi
$$

and, when $x$ is real, $U(A, X, \vartheta)$ all $v \in U$ such that

$$
v(\xi) \in \Gamma_{\xi}(A, \vartheta) \cap \operatorname{Re} X, \quad \forall \xi .
$$

Let $V(A, \vartheta) \subset U(A, \vartheta)$ and $V(A, X, \vartheta) \subset U(A, X, \vartheta)$ be subfamilies characterized by

$$
0<t \leqslant 1 \Rightarrow a(\xi \pm i t v(\xi)) \neq 0 .
$$

Note that, since $\Gamma_{\xi}(A, \vartheta)=\Gamma\left(A_{\xi}, \vartheta\right)$ only depends on the double ray $\dot{R} \xi$, (6.1) and (6.3) are consistent. By (5.16), the right side of (6.4) is never empty when $x$ is outside $\pm W(A, \vartheta)$. It is clear that $U$ is linear and, since the right sides of $(6.3),(6.4)$ are convex cones, $U(A, \vartheta)$ and $U(A, X, \vartheta)$ are also convex cones, i.e. they admit linear combinations $s u+t v, s \geqslant 0$, $t \geqslant 0, s+t>0$.

6.6. Example. Let $\gamma(\xi)$ be any positive and absolutely homogeneous $C^{\infty}$-function on $\operatorname{Re} \dot{Z}$, e.g. $\gamma(\xi)=|\xi|$. Then $v(\xi)=\gamma(\xi) \eta$ belongs to $V(A, \vartheta) \subset U(A, \vartheta)$ when $\eta \in \Gamma(A, \vartheta)$. In fact, (6.1), (6.3) hold and, since $a(\xi \pm i t \gamma(\xi) \eta)=\gamma(\xi)^{-m} a\left(\xi \gamma(\xi)^{-1} \pm i t \eta\right) \neq 0$ for all real $t \neq 0$, (6.5) is also satisfied. If $x \eta=0$, then $v(\xi) \in V(A, X, \vartheta)$.

Two elements $v_{0}$ and $v_{1}$ in any of our families, say $T$, are said to be homotopic if there is a function $[0,1] \ni s \rightarrow w_{s} \in T$ such that $s, \xi \rightarrow w_{s}(\xi)$ is infinitely differentiable and $w_{0}=v_{0}$, $w_{1}=v_{1}$. Since $\Gamma_{\xi}(A, \vartheta)=\operatorname{Re} Z$ when $\xi \bar{\epsilon} \operatorname{Re} A$, any $w \in T$ is homotopic to an element of $T$ which vanishes outside a given conical neighbourhood of $\operatorname{Re} A$. We say that $T$ is open if $w \in T$ and $v \in U$ implies that $w+\varepsilon v \in T$ when $\varepsilon$ is sufficiently small. The important families are of course $V(A, \vartheta)$ and $V(A, X, \vartheta)$. As shown by the following lemma, they consist of the small elements of $U(A, \vartheta)$ and $U(A, X, \vartheta)$. The lemma is a routine consequence of Lemma 5.1 and the inner continuity of the function $a, \xi \rightarrow \Gamma_{\xi}(A, \vartheta)$. 
6.7. Lемм. Let $a \in \mathrm{Hyp}(\vartheta)$. Then

a) $U(A, \vartheta)$ is not empty and $U(A, X, \vartheta)$ is not empty when $x \bar{\epsilon} \pm W(A, \vartheta)$. Both contain elements that vanish outside arbitrarily small conical neighbourhoods of $\operatorname{Re} A$.

b) If $U_{0}(A, \vartheta)$ and $U_{0}$ are compact parts of $U(A, \vartheta)$ and $U$ respectively, then $U_{0}(A, \vartheta)+$ ${ }_{\varepsilon} U_{0} \subset U(B, \vartheta)$ and $s U_{0}(A, \vartheta)+\varepsilon U_{0} \subset V(B, \vartheta)$ provided $s>0$ and $\varepsilon$ are small enough and $b \in \mathrm{Hyp}(\vartheta)$ is sufficiently close to $a$.

c) The families $U(A, \vartheta), V(A, \vartheta)$ are open and connected; the families $U(A, X, \vartheta)$ and $V(A, X, \vartheta)$ are connected. Any compact part of $V(A, \vartheta)$ or $V(A, X, \vartheta)$ belongs to $V(B, \vartheta)$ and $V(B, X, \vartheta)$ respectively when $b \in \mathrm{Hyp}(\vartheta)$ is sufficiently close to $a$.

d) Let $x \bar{\epsilon} \pm W(A, \vartheta)$. Any $v \in V(A, \vartheta)$ such that

$$
v(\xi) \in \Gamma_{\xi}(A, \vartheta) \cap \operatorname{Re} X
$$

when $\xi$ is in some conical neighbourhood of $\operatorname{Re} X$ is homotopic in $V(A, \vartheta)$ to a $w \in V(A, X, \vartheta)$ under a homotopy that respects (6.8). There exists $a v \in V(A, \vartheta)$ such that $x v(\xi)$ has a given constant sign for all $\xi \in \operatorname{Re} \dot{Z}$.

Proof. We give a general construction of elements in $U(A, \vartheta)$. Let $\zeta \in \operatorname{Re} \dot{Z}$ and choose an $\eta \in \Gamma_{\zeta}(A, \vartheta)$. Then, by Lemma 5.9, all positive multiples of $\eta$ belong to $\Gamma_{\xi}(A, \vartheta)$ when $\xi$ is close enough to $\zeta$ and, since $\Gamma_{\xi}(A, \vartheta)$ only depends on the double ray $\dot{R} \xi$, the same holds if $\xi$ belongs to a small enough conical neighbourhood of $\dot{R} \zeta$. Hence there is a function $0 \leqslant \varphi(\xi) \in C(\operatorname{Re} \dot{Z})$ satisfying $(6.1)$ such that $\varphi(\xi) \eta \in \Gamma_{\xi}(A, \vartheta)$ when $\xi$ is in a small enough conical neighbourhood of $\dot{R} \zeta$ while $\varphi(\xi) \eta \in \Gamma_{\xi}(A, \vartheta) \cup\{0\}$ otherwise. Covering Re $\dot{Z}$ by a finite number of such neighbourhoods and adding the corresponding vector fields, we get an element of $U(A, \vartheta)$. When $x \bar{\epsilon} \pm W(A, \vartheta)$, then, by (5.16), $\Gamma_{\xi}(A, \vartheta) \cap \operatorname{Re} X$ is never empty and, chosing $\eta$ in this set, the construction gives an element of $U(A, X, \vartheta)$. This proves the first part of a). The second follows from the fact that $\Gamma_{\xi}(A, \vartheta)=\operatorname{Re} Z$ when $\xi$ is outside Re $A$. It suffices to verify b) for vector fields restricted to, e.g., the sphere $|\xi|=1$. In fact, if (6.3), (6.4), (6.5) hold for such $\xi$, by virtue of (6.1) and the homogeneity of $a$, they hold for all $\xi$. Let $M(\xi)$ and $N(\xi)$ be the values of $v(\xi)$ and $w(\xi)$ for $|\xi|=1$ and $v \in U_{0}(A, \vartheta)$, and $w \in U_{0}$ respectively. Then $M(\xi) \subset \Gamma_{\xi}(A, \vartheta)$ and $N(\xi)$ are compact sets and the functions $\xi \rightarrow M(\xi)$ and $\xi \rightarrow N(\xi)$ are outer continuous. Normalize $a$ and $b$ such that $a(\vartheta)=b(\vartheta)=1$. Fix an $\eta$ with $|\eta|=1$ and let $M^{\prime}(\eta) \subset \Gamma_{\eta}(A, \vartheta)$ be a compact neighbourhood of $M(\xi)$. Then $M(\xi)+\varepsilon N(\xi) \subset M^{\prime}(\eta)$ if $\xi-\eta$ and $\varepsilon>0$ are small enough and, by the inner continuity of $\xi, b \rightarrow \Gamma_{\xi}(B, \vartheta), M^{\prime}(\eta) \subset \Gamma_{\xi}(B, \vartheta)$ if $\xi-\eta$ and $b-a$ are small enough. Hence $M(\xi)+\varepsilon N(\xi) \subset$ $\Gamma_{\xi}(B, \vartheta)$ if $\xi-\eta, \varepsilon>0$ and $b-a$ are small enough. Covering $|\xi|=1$ with a finite number of 11 - 702906 Aeta mathematica. 124. Imprimé le 9 Avril 1970. 
such neighbourhoods, we have $M(\xi)+\varepsilon N(\xi) \subset \Gamma_{\xi}(B, \vartheta)$ for all $\xi$ with $|\xi|=1$ when $\varepsilon>0$ and $b-a$ are small enough. This proves the first part of $\mathbf{b})$. To prove the second part, note that, by Lemma 5.1, $b\left(\xi+i s M^{\prime}(\eta)\right) \neq 0$ and hence also $b(\xi+i s(M(\xi)+\varepsilon N(\xi))) \neq 0$ when $\xi-\eta, b-a, \varepsilon>0$ and $s>0$ are small enough. A covering argument shows that the same statement holds for all $\xi$ with $|\xi|=1$ when $b-a, \varepsilon>0$ and $s>0$ are small enough and this means that under the same hypothesis $s U_{0}(A, \vartheta)+s \varepsilon U_{0} \subset V(B, \vartheta)$. This proves the second part of b). That $U(A, \vartheta)$ is open follows immediately from b). If $v \in V(A, \vartheta)$ and $U_{0}$ is a compact part of $U$ then, by b), there are positive numbers $\varepsilon_{0}, s_{0}, \delta_{0}$ such that $v(\xi)+\varepsilon N(\xi) \subset \Gamma_{\xi}(A, \vartheta)$ and $b(\xi+i s(v(\xi)+N(\xi))) \neq 0$ for all $\xi$ with $|\xi|=1$ when $0 \leqslant \varepsilon \leqslant \varepsilon_{0}, 0<s \leqslant s_{0},|b-a| \leqslant \delta_{0}$. On the other hand, since $v \in V(A, \vartheta), a(\xi+i s v(\xi)) \neq 0$ for all $\xi$ with $|\xi|=1$ when $s_{0} \leqslant s \leqslant 1$. It follows that there is an $0<\varepsilon_{1} \leqslant \varepsilon_{0}$ such that, under the same hypothesis, $a(\xi+i s(v(\xi)+$ $\varepsilon N(\xi))) \neq 0$ when $0 \leqslant \varepsilon \leqslant \varepsilon_{1}$. Here the arguments of $a$ constitute a compact set and hence there is a $0<\delta_{1} \leqslant \delta_{0}$ such that also $b(\xi+i s(v(\xi)+\varepsilon N(\xi))) \neq 0$ for all $\xi$ with $|\xi|=1$ when $s_{0}<s \leqslant 1,0 \leqslant \varepsilon \leqslant \varepsilon_{1},|b-a| \leqslant \delta_{1}$, But the inequality is true also when $0<s \leqslant s_{0}$ and this shows that $v+\varepsilon U_{0} \subset V(A, \vartheta)$ when $\varepsilon$ is small enough. Hence $V(A, \vartheta)$ is open. Since both $U(A, \vartheta)$ and $U(A, X, \vartheta)$ are positive cones, they are connected. If $t \rightarrow v_{t}$ is a homotopy in $U(A, \vartheta)$ or $U(A, X, \vartheta)$, then by $\mathrm{b}), t \rightarrow s v_{t}$ is a homotopy in $V(A, \vartheta)$ and $V(A, X, \vartheta)$ respectively when $s>0$ is small enough and hence $V(A, \vartheta)$ and $V(A, X, \vartheta)$ are connected. If $V_{0}(A, \vartheta)$ is a compact part of $V(A, \vartheta)$, then by b) there is a $0<s_{0} \leqslant 1$ such that $s_{0} V_{0}(A, \vartheta) \subset V(B, \vartheta)$ when $b$ is close enough to $a$. But $a(\xi+i s v(\xi)) \neq 0$ when $s_{0} \leqslant s \leqslant 1, v \in V_{0}(A, \vartheta)$ and $|\xi|=1$ implies the same inequality when $a$ is replaced by $b$ and $b$ is close enough to $a$. [Hence $V_{0}(A, \vartheta \subset V(B, \vartheta)$ when $b$ is close enough to $a$. The same argument shows that any compact part of $V(A, X, \vartheta)$ is contained in $V(B, X, \vartheta)$ when $b$ is close enough to $a$. This proves c). By Lemma 3.52, $L_{\xi}(A, \vartheta)+R \xi=\Gamma_{\xi}(A, \vartheta)$ when $\xi \in \operatorname{Re} \dot{Z}$. Hence, if $v \in U(A, \vartheta)$ has the property $(6.8)$, then $v_{t}(\xi)=v(\xi)-t x v(\xi)(x \xi)^{-1} \xi, 0 \leqslant t \leqslant 1$, is a homotopy in $U(A, \vartheta)$ from $v$ to $w=v_{1} \in U(A, X, \vartheta)$ that respects $(6.8)$. If $v \in V(A, \vartheta)$, then by a previous argument, the homotopy $t \rightarrow s v_{t}(\xi)$ from $s v$ to $s w$ takes place in $V(A, \vartheta)$ when $s>0$ is small enough. Hence the first part of $d$ ) follows. To prove the last part take $v_{0} \in V(A, X, \vartheta)$ and choose $u \in U$ such that $x u(\xi)$ has a constant "sign. Then, since $V(A, \vartheta)$ is open $v=v_{0}+\varepsilon u \in V(A, \vartheta)$ when' $\varepsilon>0$ is small enough and $x v(\xi)=\varepsilon x u(\xi)$ has the sign of $x u(\xi)$. This finishes the proof.

Cycles. We are going to associate to our vector fields cycles and relative cycles in complex projective space. In the following, all homology and cohomology is over the complex numbers and all homology has compact supports and all cohomology has arbitrary supports. Further, with $Z=\mathbf{C}^{n}$, let $Z^{+}=\dot{Z} / R^{+}$and $Z^{*}=\dot{Z} / \dot{\mathbf{C}}$ be the quotients of $\dot{Z}$ by the 
positive real numbers and the non-zero complex numbers respectively. In particular, $Z^{*}$ is $(n-1)$-dimensional complex projective space, while $Z^{+}$is the $(2 n-1)$-sphere. When $B \subset Z$, let $B^{+}$and $B^{*}$ be the images of $\dot{B}$ in $Z^{+}$and $Z^{*}$ respectively.

Our cycles will be oriented with the aid of the differential form on $Z$,

$$
\omega(\xi)=\sum_{1}^{n} \xi_{j} \tau_{j}(\xi)
$$

where $\tau_{f}(\xi)$ is the right cofactor of $d \xi_{j}$ in $d \xi=d \xi_{1} \wedge \ldots \wedge d \xi_{n}$ so that $d \xi=d \xi_{j} \wedge \tau_{j}(\xi)$. The corresponding form $\omega_{x}(\xi)$ on the hyperplane $X: x \xi=0,(x \neq 0)$, is defined by

$$
\omega(\xi)=d(x \xi) \wedge \omega_{x}(\xi)+O(x \xi) .
$$

Now, when $x$ is real, let $\beta(x)^{+}$be the $(n-1)$-sphere $\operatorname{Re} Z^{+}$counted with the multiplicity $\frac{1}{2}$ and oriented by

$$
x \xi \omega(\xi)>0 .
$$

This makes $\beta(x)^{+}$a cycle of $\left(\operatorname{Re} Z^{+}, \operatorname{Re} X^{+}\right)$; its boundary

$$
\partial \beta(x)^{+}=\operatorname{Re} X^{+}
$$

is an $(n-2)$-sphere with the orientation

$$
\omega_{x}(\xi)>0
$$

induced by $(6.11)$ and $d(x \xi)>0$.

6.14. Definition. When $a \in \operatorname{Hyp}(\vartheta), x \bar{\epsilon} \pm W(A, \vartheta)$ is real and $v \in V(A, X, \vartheta)$, let

$$
\alpha^{*}=\alpha(A, x, \vartheta)^{*}, \quad \bar{\alpha}^{*}=\bar{\alpha}(A, x, \vartheta)^{*} \in H_{n-1}\left(Z^{*}-A^{*}, X^{*}\right)
$$

be the homology classes of the images of $\beta(x)^{+}$in $Z^{*}$ under the maps

$$
\xi \rightarrow \xi \mp i v(\xi), \quad \xi \in \operatorname{Re} \dot{Z} .
$$

Note. In a different form and for regular $\operatorname{Re} A$, the class $\alpha^{*}$ has been used by Leray (1962) in his work on the general Laplace transform (1.c. p. 140).

The classes $\alpha^{*}, \bar{\alpha}^{*}$ are represented by the images $\alpha_{v}^{*}, \bar{\alpha}_{v}^{*}$ in $Z^{*}-A^{*}$ of $\beta(x)^{+}$under (6.16), oriented by $(6.11)$. Since $v(\xi) \in X$ for all $\xi$, these images change their orientation on $X^{*}$. The classes

$$
\partial \alpha^{*}, \partial \bar{\alpha}^{*} \in H_{n-2}\left(X^{*}-X^{*} \cap A^{*}\right)
$$

11* - 702909. Acta mathematica. 124. Imprimé le 7 Avril 1970. 
are represented by the images $\partial \alpha_{v}^{*}, \partial \bar{\alpha}_{v}^{*}$ in $X^{*}-A^{*} \cap X^{*}$ of $\operatorname{Re} X^{+}$under (6.16), oriented by (6.13).

By Lemma 6.7, $V(A, X, \vartheta)$ is one homotopy class and hence the homology classes of $\alpha_{v}^{*}, \bar{\alpha}_{v}^{*}$ do not depend on the choice of $v \in V(A, X, \vartheta)$ and this justifies the notation (6.15).

Let $\alpha_{v}^{+}, \bar{\alpha}_{v}^{+}$be the images of $\beta(x)^{+}$in $Z^{+}$under the maps (6.16) and let $\iota: \xi \rightarrow-\xi$ be the antipodal map $Z^{+} \rightarrow Z^{+}$. Then, by (6.11), (6.13) and the absolute homogeneity (6.1) of $v$,

$$
\iota\left(\bar{\alpha}^{+}\right)=(-1)^{n-1} \alpha^{+}, \quad \iota\left(\partial \bar{\alpha}^{+}\right)=(-1)^{n-1} \partial \alpha^{+}
$$

so that

$$
\begin{array}{cl}
\bar{\alpha}^{*}=(-1)^{n-1} \alpha^{*}, & \partial \bar{\alpha}^{*}=(-1)^{n-1} \partial \alpha^{*} \\
2 \alpha^{*} \ni \alpha_{v}^{*}+(-1)^{n-1} \bar{\alpha}_{v}^{*}, & 2 \partial \alpha^{*} \ni \partial \alpha_{v}^{*}+(-1)^{n-1} \partial \bar{\alpha}_{v}^{*} .
\end{array}
$$

Note also that

$$
x \bar{\epsilon} \pm K(A, \vartheta) \Rightarrow \alpha^{*}=0 \text { in } H_{n-1}\left(Z^{*}-A^{*}, X^{*}\right)
$$

In fact, then there exists an $\eta \in \Gamma(A, \vartheta)$ such that $x \eta=0$ and, putting $v(\xi)=|\xi| \eta$, the homotopy $\xi-i|\xi| \eta \rightarrow t \xi-i|\xi| \eta$ where $0 \leqslant t \leqslant 1$, contracts $\alpha_{v}^{+}$in $Z^{+}-A^{+}$to the point $(-i|\xi| \eta)^{+}$in $X^{+}$. We also note in passing that

$$
n \text { even, } \operatorname{Re} \dot{X} \cap A=\varnothing \Rightarrow \partial \alpha^{*}=0 \text { in } H_{n-2}\left(X^{*}-X^{*} \cap A^{*}\right) .
$$

This follows from (6.20) if we choose $v \in V(A, X, \vartheta)$ equal to zero in a small conical neigh. bourhood of $\operatorname{Re} X$.

The following lemma summarizes some useful continuity properties of the homology class $\alpha(A, x, \vartheta)^{*}$.

6.23. Lемм. Let $a \in \operatorname{Hyp}(\vartheta), x \vec{\epsilon} \pm W(A, \vartheta), v \in V(A, X, \vartheta)$. Then

a) the class $\alpha(A, x, \vartheta)^{*}$ contains every cycle $\alpha_{w}^{*}$ for which $w \in V(A, \vartheta)$ and $w(\xi) \in \operatorname{Re} X$ when $\xi$ belongs to an arbitrarily small conical neighbourhood of $\operatorname{Re} X$.

b) If $b \in \operatorname{Hyp}(\vartheta)$ and $y \in \operatorname{Re} Z$ are close enough to $a$ and $x$ respectively, we have a natural commutative diagram

$$
\begin{array}{cc}
H_{n-1}\left(Z^{*}-A^{*}\right) \rightarrow H_{n-1}\left(Z^{*}-A^{*}, X^{*}\right) \rightarrow H_{n-2}\left(X^{*}-X^{*} \cap A^{*}\right) \\
\downarrow \varphi \quad \downarrow \varphi \\
H_{n-1}\left(Z^{*}-B^{*}\right) \rightarrow H_{n-1}\left(Z^{*}-B^{*}, Y^{*}\right) \rightarrow H_{n-2}\left(Y^{*}-Y^{*} \cap B^{*}\right),
\end{array}
$$

where $\varphi \alpha(A, x, \vartheta)^{*}=\alpha(B, y, \vartheta)^{*}$. 
Note. As we shall see in part II, $\alpha(A, x, \vartheta)^{*} \neq 0$ in $H_{n-1}\left(Z^{*}-A^{*}, X^{*}\right)$ when $x \in K(A, \vartheta)-$ $W(A, \vartheta)$.

Proof. a) follows from Lemma 6.7d. The homomorphisms $\varphi$ and $\psi$ of the diagram of b) are obtained by using projection onto $Y^{*}$. We indicate the details. Choose an $\eta \in \operatorname{Re} Z$ such that $x \eta \neq 0$ and restrict $y$ to a ball around $x$ where $y \eta \neq 0$ and let

$$
p=p(Y): \zeta \rightarrow \zeta-(y \zeta)(y \eta)^{-1} \eta
$$

be the projection on $Y$ along $\eta$. Further, let $r=r(Y)$ be the trace of this projection, i.e. the line segment $(1-t) \zeta+t p \zeta, 0 \leqslant t \leqslant 1$, oriented by $d t>0$. The corresponding maps in projective space, defined on $Z^{*}-\eta^{*}$ will be denoted by $p^{*}$ and $r^{*}$ respectively. When $c^{*} \subset Z^{*}-\eta^{*}$ is a compact oriented chain, then $r^{*} c^{*}$, suitably oriented by the product orientation is a compact chain in $Z^{*}-\eta^{*}$ such that

$$
\partial r^{*} c^{*}=p^{*} c^{*}-c^{*}
$$

Let us also put

$$
s^{*} c^{*}=\partial c^{*}+r^{*} \partial c^{*}
$$

so that, if $\partial c^{*} \subset X^{*}$, then $\partial s^{*} c^{*} \subset Y^{*}$. The maps $\psi$ and $\varphi$ are then induced by $p^{*}$ and $s^{*}$ respectively. The verification that the diagram is well defined and commutative when $y, b$ are close to $x, a$ is left to the reader. The following figure illustrates the construction.

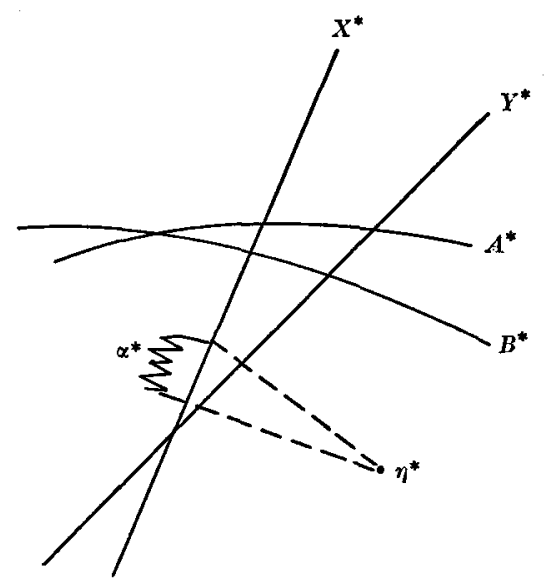

Fig. 6

It remains to verify that $\varphi \alpha(A, x, \vartheta)^{*}=\alpha(B, y, \vartheta)^{*}$. Consider the chain

$$
c(x, y)=r \operatorname{Re} \dot{X}
$$

where each point counts once and $c(x, y)^{+}$is oriented by $d t \wedge \omega_{x}(\xi)>0$. We shall see that 


$$
c(x, y)^{+}=\beta(y)^{+}-\beta(x)^{+} .
$$

In fact, the points of $c(x, y)$ are

$$
p_{t} \xi=(1-t) \xi+t p \xi=\xi-t(y \xi)(y \eta)^{-1} \eta, \quad \xi \in \operatorname{Re} \dot{X}, \quad 0 \leqslant t \leqslant 1,
$$

and its boundary consists of $\operatorname{Re} \dot{X}$ and $\operatorname{Re} \dot{Y}$. Since $\operatorname{sgn} x \xi_{t}=-t \operatorname{sgn} y \xi$ and $\operatorname{sgn} y \xi_{t}=$ $(1-t) \operatorname{sgn} y \xi$, the interior points $\zeta$ have the property that sgn $y \zeta-\operatorname{sgn} x \zeta \neq 0$. Conversely, if $\zeta$ is such a point, $\zeta$ is a positive combination of the points on the line $s \rightarrow \zeta+s \eta$ where it meets $\operatorname{Re} X$ and $\operatorname{Re} Y$ respectively. Hence $c(x, y)^{+}$and $\beta(y)^{+}-\beta(x)^{+}$have the same carrier. Now the points of $\beta(y)^{+}-\beta(x)^{+}$have the multiplicity $\frac{1}{2}(\operatorname{sgn} y \zeta-\operatorname{sgn} x \zeta)$ and hence (6.24) holds except for a possible factor \pm 1 . We leave it to the reader to verify that the orientations $d t \wedge \omega_{x}(\xi)>0,(\xi \in \operatorname{Re} \dot{X})$, and ( $\left.\operatorname{sgn} y \zeta-\operatorname{sgn} x \zeta\right) \omega(\zeta)>0$ coincide on $c(x, y)^{+}$. Next, by definition, $r^{+} \partial \alpha_{v}^{+}$has the real projection (6.24) and its points are

$$
\left\{p_{t} \xi-i p_{t} v(\xi)\right\}^{+}, \quad \xi \in c(x, y), \quad 0 \leqslant t \leqslant 1 .
$$

Further, $\alpha_{v}^{+}$has the real projection $\beta(x)^{+}$and its points are

$$
\frac{1}{2}\{\xi-i v(\xi)\}^{+} .
$$

The boundary of $s^{+} \alpha_{v}^{+}=\alpha_{v}^{+}+r^{+} \partial \alpha_{v}^{+}$has the real projection $\operatorname{Re} Y^{+}$, its points

$$
\{p \xi-i p v(\xi)\}^{+}, \quad \xi \in \operatorname{Re} \dot{X}
$$

belong to $Y^{+}$. Small continuous deformations of $s^{+} \alpha_{v}^{+}$that carry $Y^{+}$into $Y^{+}$do not change the class of $s^{+} \alpha_{v}^{+}$in $\left(Z^{+}-A^{+}, Y^{+}\right)$. Replacing the imaginary parts of (6.25), (6.25) by $p v\left(p_{t} \xi\right)$ and $p v(p \xi)$ is the result of such a deformation when $y$ is close enough to $x$. But, in view of (6.24), this changes $s^{+} \alpha_{v}^{+}$to $\alpha_{p v}^{+}$. Since $V(A, \vartheta)$ is open, it contains $p v$ when $y$ is close enough to $x$ and hence $\alpha_{p y}^{*} \in \alpha(A, y, \vartheta)^{*}$. The whole argument also permits small changes of $a$ within the class Hyp $(\vartheta)$ and this finishes the proof of $b$ ).

It follows from (6.19) that $\alpha^{*}$ depends drastically on the parity of $n$. We shall illustrate this further by some examples.

Example, $n$ even. Let us first consider the case $n=2$. Choose a basis $\eta^{\prime}, \eta^{\prime \prime}$ of $Z$ such that $\eta^{\prime} \in \Gamma(A, \vartheta)$ and $\eta^{\prime \prime} \in \operatorname{Re} X$ and let $\xi=u \eta^{\prime}+v \eta^{\prime \prime}$ define coordinates $u, v$ in $Z$. Taking $v=1$, we may represent $Z^{*}$ by the complex $u$-plane $U$ with a point at infinity and then $X^{*}$ is represented by the origin of $U$ and $A^{*}$ by a finite number of points of $\operatorname{Re} U$ disjoint from zero and infinity while $\Gamma(A, \vartheta)^{*}$ is represented by the component of infinity in $\operatorname{Re} U-A^{*}$. Choosing the vector field $v(\xi)=0$ off a small conical neighbourhood of $\operatorname{Re} A$, it follows from (6.12), (6.13), (6.20) that $2 \alpha^{*}$ is represented by the boundaries of small disks 
centered at the image of $A^{*}$ and oriented by the orientation of $U$ multiplied by the sign of $x \xi$ taken at their centers. All these orientations are the same if and only if $X^{*}$ meets $\Gamma(A, \vartheta)^{*}$, i.e. if and only if $x \bar{\epsilon} \pm K(A, \vartheta)$. This is now also a necessary condition for $\alpha^{*}$ to vanish.

Many of the features of the case $n=2$ subsist when $n>2$ is even. It follows for instance from (6.20) and the definition of $V(A, X, \vartheta)$ that $\alpha^{*}$ is represented by a tube around $\operatorname{Re} A^{*}$, i.e. the locus of the boundary of a small two-dimensional disk whose center moves on $\operatorname{Re} A^{*}$. The boundary of the disk is outside $A^{*}$ and the disk should belong to $X^{*}$ when its center has that property. By (6.13), the orientation changes when the center of the disk passes $\operatorname{Re} A^{*} \cap$ $\operatorname{Re} X^{*}$. The class $\partial \alpha^{*}$ is represented by a tube around $\operatorname{Re} A^{*} \cap X^{*}$, suitably oriented. Typically, $\operatorname{Re} A^{*} \cap X^{*}$ is a set of $(n-3)$-dimensional ovals with certain orientations. When $A^{*} \cap X^{*}$ is non-singular, then as will be shown in Part II, $\partial \alpha^{*}=0$ in $H_{n-2}\left(X^{*}-X^{*} \cap A^{*}\right)$ means that $\operatorname{Re} A^{*} \cap X^{*}$ is homologous to zero in $A^{*} \cap X^{*}$. (6.22) provides a trivial example. Petrovsky (1945) p. 349-350 gives a non-trivial example when $m=4$. When $n=4$, then $A^{*} \cap X^{*}$ is just an algebraic curve.

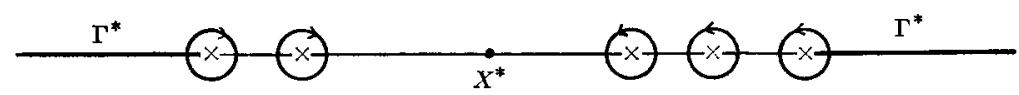

Fig. 6 a. $x=2$. Crosses indicate $A^{*}$, circles $2 \alpha^{*} . \partial \alpha^{*}$ vanishes.

Example, $n$ odd. Let us first take the case $n=3$. Let $\eta^{\prime}, \eta^{\prime \prime}$ be real and span $X$ and let $\xi=u \eta^{\prime}+v \eta^{\prime \prime}$ define coordinates in $X$. Taking $v=1$, we may represent $X^{*}$ as a complete complex plane $U$. When $x \bar{\epsilon} \pm W(A, \vartheta)$, then $a$ is not identically zero on $\operatorname{Re} X$. In fact, if this were the case, $a(\zeta)$ must have the factor $x \zeta$ so that, if $\xi \in \operatorname{Re} X, \Gamma_{\xi}(A, \vartheta)$ is contained in $\Gamma_{\xi}(X, \vartheta)$ which is a half-space not containing $\operatorname{Re} X$ and this contradicts the requirement $\Gamma_{\xi}(A, \vartheta) \cap \operatorname{Re} X \neq \varnothing$. Hence we can choose $\eta^{\prime}$ such that $a\left(\eta^{\prime}\right) \neq 0$ and then $A^{*} \cap X^{*}$ is represented by a number of finite real points in $U$ together with a number of finite complex conjugate pairs. Further, choosing a $v(\xi) \in V(A, X, \vartheta)$ which vanishes off a small neighbourhood of $\operatorname{Re} A$, by virtue of (6.14), (6.15), (6.20), 22 $\alpha^{*}$ may be represented by twice $\operatorname{Re} U$ detached from the image of $\operatorname{Re}\left(A^{*} \cap X^{*}\right)$ as shown in figure $6 \mathrm{~b}$. According to (6.15), the orientation of $\partial \alpha^{*}$ is that induced by $\operatorname{Re} X^{*}$. Note that $2 \partial \alpha^{*}$ is homologous in $X^{*}-A^{*} \cap X^{*}$ to tubes around the non-real part of $A^{*} \cap X^{*}$ oriented by the sign of $\operatorname{Im} u$. They are marked by dotted circles in the figure. Hence, if $n=3$, we have

$$
\partial \alpha^{*}=0 \Leftrightarrow A^{*} \cap X^{*} \text { real. }
$$

In the figure $5 \mathrm{~b}$, points with this property are either outside $K(A, \vartheta)$ or, except in the case 8 , inside a curved triangle. 
The main features of figure $5 \mathrm{~b}$ subsist when $n>3$ is odd. In particular, by (6.12) and (6.20), $\alpha^{*}$ and $\partial \alpha^{*}$ may be represented by $\operatorname{Re} Z^{*}$ detached from $\operatorname{Re} A^{*}$ and $2 \operatorname{Re} X^{*}$ detached from $\operatorname{Re} A^{*} \cap X^{*}$ respectively. In the first case, the orientation changes on $X^{*}$.

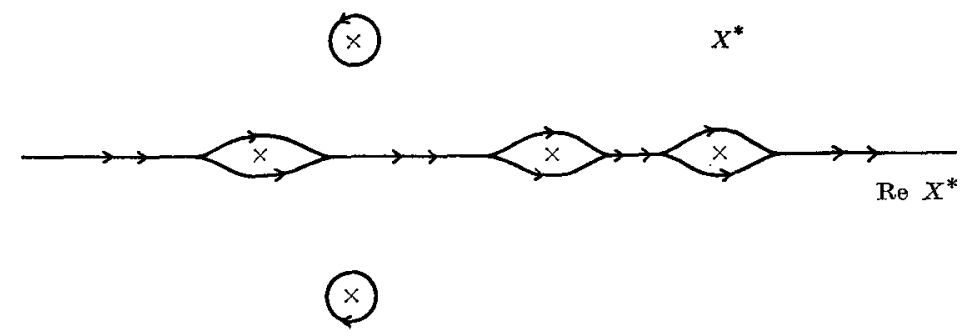

Fig. 6 b. $n=3$. Crosses indicate $A^{*} \cap X^{*}$, lines $2 \partial \alpha^{*}$.

It is easy to generalize one part of (6.26) to higher dimensions. This is done by the following theorem suggested by an example of Petrovsky (1945, p. 348).

6.27. ТнEоR см. Let $n>1$ be odd and let $a_{x}$ be the restriction of $a \in \mathrm{Hyp}(\vartheta)$ to $X$. If $x \bar{\epsilon} \pm W(A, \vartheta)$ and there is $a \vartheta_{x} \in \operatorname{Re} X$ such that $a_{x} \in \operatorname{Hyp}\left(\vartheta_{x}\right)$ and one of the sets $\pm \Gamma_{\xi}\left(A_{x}, \vartheta_{x}\right) \cap$ $\Gamma_{\xi}(A, \vartheta)$ is never empty when $\xi \in \operatorname{Re} \dot{X}$, then $\partial \alpha(A, x, \vartheta)^{*}=0$ in $H_{n-2}\left(X^{*}-X^{*} \cap A^{*}\right)$. When all $\Gamma_{\xi}(A, \vartheta) \cap \operatorname{Re} X$ are at least half-spaces, it suffices that $a_{x} \in \operatorname{Hyp}\left(\vartheta_{x}\right)$ for some $\vartheta_{x} \in \operatorname{Re} X$.

Proof. Since the function

$$
\operatorname{Re} \dot{X} \ni \xi \rightarrow\left(\Gamma_{\xi}\left(A_{x}, \vartheta_{x}\right) \cup-\Gamma_{\xi}\left(A_{x}, \vartheta_{x}\right)\right) \cap \Gamma_{\xi}(A, \vartheta)
$$

is inner continuous, the construction used in the proof of Lemma 6.7 shows that there exists a $v \in V(A, X, \vartheta)$ whose restriction $v_{x}$ to $\operatorname{Re} \dot{X}$ has the property that $v_{x}(\xi)$ belongs to one of the sets $\pm \Gamma_{\xi}\left(A_{x}, \vartheta_{x}\right) \subset \operatorname{Re} \dot{X}$. If $\xi \in \operatorname{Re} A$, the sign is well-defined. More precisely, there is a continuous absolutely homogeneous function $\varepsilon(\xi)= \pm 1$ defined in a conical neighbourhood $N \subset \operatorname{Re} \dot{X}$ of $\operatorname{Re} A \cap \operatorname{Re} \dot{X}$ such that $v_{x}(\xi) \in \varepsilon(\xi) \Gamma_{\xi}\left(A_{x}, \vartheta_{x}\right) \subset \operatorname{Re} \dot{X}$ for all $\xi \in N$. Taking $v_{x}$ to be zero outside $N$, which is no restriction, this gives us a vector field $w(\xi)=$ $\varepsilon(\xi) v_{x}(\xi)$ belonging to $V\left(A_{x}, \vartheta_{x}\right)$. Now $\partial \alpha^{*}=\partial \alpha(A, x, \vartheta)^{*}$ is represented by the image in projective space $X^{*}$ of the cycle $\gamma(x)^{+}=\left\{\xi-i v_{x}(\xi) ; \xi \in \operatorname{Re} \dot{X}\right\}^{+}$oriented by $\omega_{x}(\xi)>0$. Since $n$ is odd, $\omega_{x}(-\xi)=\omega_{x}(\xi)$, and hence $\partial \alpha^{*}$ is also represented by half the image of $\gamma(x)^{+}$plus its conjugate. This shows that the image in $X^{*}$ of $\gamma(x)^{+}$is the same as the image of the cycle $\gamma_{1}(x)^{+}=\{\xi-i w(\xi) ; \xi \in \operatorname{Re} \dot{X}\}^{+}$where $w=\varepsilon v_{x} \in V\left(A_{x}, \vartheta_{x}\right)$. Now all vector fields in $V\left(A_{x}, \vartheta_{x}\right)$ are homotopic and hence $\partial \alpha^{*}$ is also represented by, e.g., the cycle $\left\{\xi-i s|\xi| \vartheta_{x}\right.$; $\xi \in \operatorname{Re} X\}^{*}$ where $s>0$. Letting $s \rightarrow \infty$, this cycle contracts in $X^{*}-X^{*} \cap A^{*}$ to a point and 
hence $\partial \alpha^{*}=0$ in $H_{n-2}\left(X^{*}-X^{*} \cap A^{*}\right)$. If all $\Gamma_{\xi}(A, \vartheta) \cap \operatorname{Re} X$ are at least half-spaces (and this is true when $n=3)$, then, since $\Gamma_{\xi}\left(A_{x}, \vartheta_{x}\right)$ is an open convex cone in $\operatorname{Re} X$, at least one of the sets $\pm \Gamma_{\xi}\left(A_{x}, \vartheta_{x}\right) \cap \Gamma_{\xi}(A, \vartheta)$ is never empty. This finishes the proof.

\section{Fundamental solutions expressed as rational integrals}

We now have all the necessary tools for justifying our heuristic analysis at the end of Section 4 of the formula (4.13), i.e. in integrated form

$$
\left(E_{s}, \check{g}\right)=(2 \pi)^{-n} \int a(\xi+i \eta)^{-s} \exists g(\xi+i \eta) d(\xi+i \eta),
$$

where $g \in C_{0}\left(\operatorname{Re} Z^{\prime}\right), \check{g}(x)=g(-x)$. We are going to modify the chain of the integration in (7.1), replacing $\eta$ by a real vector field $w(\xi)$. Later we shall be able to choose $w$ in $V(A, \vartheta)$. To begin with, for reasons explained earlier, we have to work with bounded vector fields. For this reason we introduce a cut-off operation $v \rightarrow v_{r}$ defined for absolutely homogeneous vector fields by

$$
v_{r}(\xi)=\min (r / \gamma(\xi), 1) v(\xi), \quad r>0,
$$

where $0<\gamma(\xi) \in C(\operatorname{Re} Z)$ satisfies (6.1). We could choose $\gamma(\xi)=|\xi|$; our more general choice will be useful later on. Our next theorem uses the differential $(n-1)$-form $\omega(\xi)$ defined by (6.9). It has the properties that

$$
\begin{gathered}
d\left(g f_{1}\right) \wedge \ldots \wedge d\left(g f_{n}\right)=g^{n} d f_{1} \wedge \ldots \wedge d f_{n}+g^{n-1} d g \wedge \omega\left(f_{1}, \ldots, f_{n}\right) \\
d(h(\xi) \omega(\xi))=\left(\sum \xi_{k} \partial h / \partial \xi_{k}+n h\right) d \xi_{1} \wedge \ldots \wedge d \xi_{n},
\end{gathered}
$$

where $g, f, \ldots$ are functions and $h(\xi)$ a holomorphic function. Note that the first term on the right of (7.3) vanishes when $f_{1}, \ldots, f_{n}$ are dependent and that the right side of (7.4) vanishes when $h(\xi)$ is homogeneous of degree $-n$. The theorem also employs the holomorphic functions and distributions $\chi_{s}, \chi_{s}^{0}$, defined by (1.6), (1.9), (1.11), (1.14).

7.5. ТнEоR ем. Let $a \in \operatorname{Hyp}(\vartheta, m), v \in V(A, \vartheta), g \in C_{0}\left(\operatorname{Re} Z^{\prime}\right)$.

a) When $\operatorname{Re} s<0$, then

$$
\left(E_{s}, \check{g}\right)=(2 \pi)^{-n} \int_{\mathrm{Re} Z} a(\zeta)^{-s} \mathcal{\exists} g(\zeta) d \zeta, \quad \zeta=\xi-i v_{r}(\xi),
$$

for all $r>0$. 
b) When $x v(\xi) \leqslant 0$ for all $\xi \in \operatorname{Re} Z$ and all $x \in \mathbb{S}(\check{g})$, the same statement holds with $v_{r}$ replaced by $v$.

c) The functions $s, x \rightarrow E_{s}(a, \vartheta, x)$ are holomorphic when $x$ is outside $W(A, \vartheta)$ and, if $x$ is outside $\pm W(A, \vartheta)$, then

$$
\begin{aligned}
E_{s}(a, \vartheta, x)= & (2 \pi)^{-n} i^{m s-n} \int_{\mathcal{\gamma}(\xi)-1} \chi_{m s-n}(x \zeta) a(\zeta)^{-s} \omega(\zeta) \\
& -(2 \pi)^{-n} \int_{\mathcal{\gamma}(\xi)-1} \chi_{m s-n}^{0}(i x \zeta) a(\zeta)^{-s}\left(\frac{1}{2} \pi i-m^{-1} \log a(\zeta)\right) \omega(\zeta),
\end{aligned}
$$

where $\zeta=\xi-i v(\xi)$ with $v \in V(A, \vartheta)$ chosen so that $x v(\xi)<0$ for all $\xi$.

d) The formula (7.7) is true also when $v \in V(A, X, \vartheta)$, provided the integrations are taken in the distribution sense. Differentiation under the integration signs is permitted.

Note. The second term of (7.7) vanishes unless $m s-n=0,1,2, \ldots$ and then it is a polynomial. The formula (7.7) is useful only when $W(A, \vartheta) \neq K(A, \vartheta)$, i.e. when $a$ is a complete polynomial. In fact, $E_{s}$ vanishes outside $K(A, \vartheta)$. When $a$ is not complete we can combine (7.7) with the formula (4.8).

Proof. For simplicity of notation, introduce the differential form

$$
\theta_{s}(\xi+i \eta)=(2 \pi)^{-n} a(\xi+i \eta)^{-8} \mathcal{\xi} g(\xi+i \eta) d(\xi+i \eta) .
$$

It is holomorphic and hence closed, and entire analytic in $s$ when $\eta \in \pm \Gamma= \pm \Gamma(A, \vartheta)$. By (2.3), to every $M>0$ there is a $c(M)<\infty$ such that

$$
|\exists g(\xi-i \eta)| \leqslant c(M)(1+|\xi-i \eta|)^{-M} e^{h(\eta)}
$$

where

$$
h(\eta)=\max x \eta, \quad x \in \mathbb{S}(\check{g}) .
$$

Also, by (4.12)

$$
\operatorname{Re} s \leqslant 0, \eta \in \Gamma \Rightarrow\left|a(\xi-i \eta)^{-s}\right| \leqslant c(s)|\xi-i \eta|^{-m \operatorname{Res}}
$$

where $c(s)>0$ is locally bounded.

First, let $\eta \in \Gamma$, put $v(\xi)=\gamma(\xi) \eta$ and consider the homotopy

$$
w_{t}(\xi)=(1-t) \eta+t v_{r}(\xi), \quad 0 \leqslant t \leqslant 1
$$

connecting $w_{0}(\xi)=\eta$ with the cut-off vector field $v_{r}(\xi)$. Combining (7.8) and (7.9) gives the following estimates of $\theta_{s}=\theta_{s}\left(\xi-i w_{t}(\xi)\right)$, 


$$
\begin{aligned}
& d t=0 \Rightarrow\left|\theta_{s}\right| \leqslant c_{M}(s)(1+|\xi|)^{-m R_{0}-M} e^{r h(\eta)}|d \xi| \\
& d \gamma(\xi)=0 \Rightarrow\left|\theta_{s}\right| \leqslant \text { the same factor times } \sum\left|d t \wedge \tau_{j}(\xi)\right|
\end{aligned}
$$

where $\tau_{j}(\xi)$ is the right cofactor of $d \xi_{j}$ in $d \xi$, so that $d \xi=d \xi_{j} \wedge \tau_{j}(\xi)$. The function $c_{M}(s)$ is locally bounded. Also, $\theta_{s}\left(\xi-i w_{t}(\xi)\right)$ is a holomorphic $(n+1)$-form on the compact chain

$$
C_{\varepsilon}=\left\{\xi-i w_{t}(\xi) ; \varepsilon \leqslant \gamma(\xi) \leqslant \varepsilon^{-1} ; 0 \leqslant t \leqslant 1\right\}
$$

and hence

$$
\int_{\partial C_{\varepsilon}} \theta_{s}=0 .
$$

By the estimates above, the integrals over the parts of $\partial C_{\varepsilon}$ where $\gamma(\xi)=\varepsilon, \varepsilon^{-1}$ tend to zero as $\varepsilon \rightarrow 0$ and the integrals over the other parts remain absolutely convergent. Hence (7.6) follows for all $r>0$ when $v(\xi)=\gamma(\xi) \eta, \eta \in \Gamma$.

Next, let $v(t, \xi), 0 \leqslant t \leqslant 1$, be a homotopy in $V(A, \vartheta)$ from $v(0, \xi)=\gamma(\xi) \eta$ to a given $v(1, \xi)=v(\xi)$ and consider the corresponding cut-off homotopy $v_{r}(t, \xi)$ from $v_{r}(0, \xi)$ to $v_{r}(1, \xi)$. We are going to estimate $\theta_{s}(\zeta), \zeta=\xi-i v_{r}(t, \xi)$, and first of all $a(\zeta)^{-s}$. Since $\operatorname{Re} s \leqslant 0$ and

$$
\left|a(\zeta)^{-s}\right|=|a(\zeta)|^{-\operatorname{Re} s} e^{\operatorname{Im} s \arg a(\zeta)}
$$

we only have to estimate $\arg a(\zeta)$. We claim that

$$
\arg a\left(\xi-i v_{r}(t, \xi)\right)
$$

is bounded when $\xi \in \operatorname{Re} \dot{Z}, 0 \leqslant t \leqslant 1$. Using the homogeneity of $a$, it suffices to consider

on the product

$$
\varphi(\xi, t, \varrho)=\arg a(\xi-i \varrho v(\xi))
$$

$$
\{\gamma(\xi)=1\} \times\{0 \leqslant t \leqslant 1\} \times\{0<\varrho \leqslant 1\} .
$$

Since $v \in V(A, \vartheta), a(\zeta) \neq 0$ for all $\xi \in \operatorname{Re} \dot{Z}$ and hence $\varphi$ is a continuous function, Clearly, $\varphi$ is bounded when $\varrho=1$, and $\varphi$ being the argument of a polynomial in $\varrho$ of degree $\leqslant m$, the variation of $\varphi$ as $0<\tau \leqslant 1$ does not exceed $m \pi$. Hence $\varphi$ is in fact bounded. By virtue of (7.10) we have the same estimates of $a(\zeta)^{-s}$ as before and hence

$$
\begin{aligned}
& d t=0 \Rightarrow\left|\theta_{s}(\zeta)\right| \leqslant c_{M}(s) \gamma(\xi)^{-m \operatorname{Re} s}(1+|\xi|)^{-M} e^{h\left(v_{r}(t, \xi)\right.}|d \xi| \\
& d \gamma(\xi)=0 \Rightarrow\left|\theta_{s}(\zeta)\right| \leqslant \text { the same factor times } \sum\left|d t \wedge \tau_{j}(\xi)\right|,
\end{aligned}
$$

where all dependence on $r$ and $t$ is displayed. Also, $\theta_{s}$ is holomorphic on the chain

$$
\left\{\xi-i v_{r}(t, \xi) ; \varepsilon \leqslant \gamma(\xi) \leqslant \varepsilon^{-1}, 0 \leqslant t \leqslant 1\right\}
$$

Hence our previous argument works and we have 11†-702906. Acta mathematica. 124. Imprimé le 9 Avril 1970. 


$$
\int \theta_{s}\left(\xi-i v_{r}(0, \xi)\right)=\int \theta_{s}\left(\xi-i v_{r}(1, \xi)\right)
$$

where both integrals are absolutely convergent. This proves a).

If $v(\xi) x \leqslant 0$ on $\mathrm{S}(\check{g})$, then $h\left(v_{r}(\xi)\right) \leqslant 0$ and the first estimate (7.11) proves that we can let $r \rightarrow \infty$ in (7.6). This proves b).

To prove $e$ ) and $(7.7)$, we first note that by Lemma $6.7 \mathrm{~d}$ ), if $x \bar{\epsilon} \pm W(A, \vartheta)$, there is a $v(\xi) \in V(A, \vartheta)$ such that $x v(\xi)<0$ for all $\xi \in \operatorname{Re} \dot{Z}$. Hence, by the homogeneity, $x v(\xi) \leqslant$ $-2 \varepsilon \gamma(\xi)$ for some $\varepsilon>0$. More generally, if $y \in \operatorname{Re} Z^{\prime}$ is close enough to $x$, say $|y-x| \leqslant \delta$, then

$$
y v(\xi)=-2 \varepsilon \gamma(\xi)+(y-x) v(\xi) \leqslant-\varepsilon \gamma(\xi) .
$$

This means that the integral

$$
F_{s}(y)=(2 \pi)^{-n} \int a(\xi-i v(\xi))^{-s} e^{i y(\xi-i v(\xi))} d(\xi-i v(\xi))
$$

is absolutely convergent when $|y-x|<\delta$ and $\operatorname{Re} s \leqslant 0$. Introducing polar coordinates, $\xi=\varrho \eta, \gamma(\eta)=1$, using (1.6) and (7.3) and reverting to $\xi$ again, we have

$$
F_{s}(y)=(2 \pi)^{-n} i^{m s-n} \int_{\gamma(\xi)=1} \chi_{m s-n}(y \zeta) a(\zeta)^{-s} \omega(\zeta),
$$

where $\zeta=\xi-i v(\xi)$. Since, by (7.12),

$$
\operatorname{Im} y \zeta \geqslant \varepsilon \gamma(\xi)
$$

and the functions $\chi$ are holomorphic in the upper half-plane, the right side of (7.14) is a holomorphic function of $y, s$ when $y$ is close to $x$ and $\chi_{m s-n}(y \zeta)$ is holomorphic in $s$. Hence, by (1.6), $F_{s}(y)$ is holomorphic in $y, s$ when $y$ is close to $x$ and $s$ is complex except perhaps for simple poles when $m s-n=0,1,2, \ldots$ On the other hand, we shall see that

$$
m s-n=0,1,2, \ldots \Rightarrow \int_{\gamma=1} \chi_{m s-n}(y \zeta) a(\zeta)^{-s} \omega(\zeta)=0
$$

which then implies that $F_{s}(y)$ is holomorphic in $s, y$ for all $s$ and all $y$ close to $x$. Assume this for the moment. Multiplying (7.13) by $g(-y)$ where $g \in C_{0}\left(\operatorname{Re} Z^{\prime}\right)$ and $|y-x|<\delta$ on $\mathrm{S}(\check{g})$, we get

$$
\left(F_{s}, \check{g}\right)=(2 \pi)^{-n} \int a(\zeta)^{-s} \varsubsetneqq g(\zeta) d \zeta, \quad \zeta=\xi-i v(\xi)
$$

By b), the right side equals $\left(E_{s}, \check{g}\right)$, which we know to be entire analytic. Hence $E_{s}(a, \vartheta, y)=$ $F_{s}(y)$ for all $y$ close to $x$ and all $s$. Since $E_{s}=E_{s}(a, \vartheta, x)$ vanishes outside $K(A, \vartheta)$ for all $s$, 
this shows that $E_{s}$ is holomorphic in $s, x$ when $x$ is outside $W(A, \vartheta)$. At the same time, we have (7.7) when $x$ is outside $\pm W(A, \vartheta)$ and $m s-n \neq 0,1,2, \ldots$. To prove (7.7) in the exceptional cases, just apply the operation

$$
\left.G_{s} \rightarrow \frac{d}{d t} t G_{s+t}\right|_{t=0}
$$

to both sides. The formula (1.13) and a small computation gives the desired result. To prove (7.15), note that $\chi_{m s-n}(x \zeta) a(\zeta)^{-s}$ is holomorphic and homogeneous of degree $-n$. Hence the integrand is a closed $(n-1)$-form. But then it is independent of the choice of $v$ in $V(A, \vartheta)$ and remains the same if we put, e.g. $v(\xi)=\gamma(\xi) \vartheta$. Next, the cycles $\{t \xi-i \gamma(\xi) \vartheta$; $\gamma(\xi)=1\}$ being homotopic in $Z-A$ when $t$ is real, letting $t \rightarrow 0$, we see that the integral vanishes. This finishes the proof of $\mathbf{c}$ ).

To prove d), take a family of vector fields $v=v_{s}$ in (7.7) such that $x v_{\varepsilon}(\xi)$ tends to zero. We may e.g. put $v(\xi)=w(\xi)-\varepsilon \gamma(\xi) \eta$ where $w \in V(A, X, \vartheta), x \eta=1$. Then, since $V(A, \vartheta)$ is open, $v \in V(A, \vartheta)$ when $\varepsilon>0$ is small enough. Then, as $\varepsilon \rightarrow 0$, the functions $\chi_{m s-n}(x \zeta)=$ $\chi_{m s-n}(x \xi+i \varepsilon)$ tend to the distribution $\chi_{m s-n}(x \xi)$ on the manifold $\gamma(\xi)=1$. The passage to the limit is legitimate by Lemma 1.2. The proof is finished since, clearly, (7.7) permits differentiation under the integration sign.

Generalization of the Herglotz-Petrovsky-Leray formulas. At long last we can now prove the formulas (4), (5) of the introduction. Our next theorem employs a tube operation $t_{x}$ from $X-X \cap A$ to $Z-A \cup X$. When $\sigma \subset X-X \cap A$ is a compact chain, let $\sigma_{r}$ be the product $\{|x \xi|=r\} \times \sigma$ with $r$ so small that $\sigma_{s} \subset Z-A$ when $0 \leqslant s \leqslant r$. When $\sigma$ is oriented, orient $\sigma_{r}$ by the product of orientation $\omega(\operatorname{Re} x \xi, \operatorname{Im} x \xi)>0$ of the complex plane with coordinate $x \xi$ and the orientation of $\sigma$. Then $t_{x} \sigma=\sigma_{\tau}$ defines a chain map from $X-X \cap A$ to $Z-A \cup X$ that increases the dimension by 1 and induces a map $t_{x}: H_{q}(X-X \cap A) \rightarrow H_{a+1}(Z-A \cup X)$ independent of the choice of $r$. We let $t_{x}$ denote also the induced map $H_{q}\left(X^{*}-X^{*} \cap A^{*}\right) \rightarrow$ $H_{q+1}\left(Z^{*}-A^{*} \cup X^{*}\right)$.

7.16. Тнеовем. Let $a \in \operatorname{Hyp}(\vartheta, m)$ and let $E(a, x)=E(a, \vartheta, x)$ be the fundamental solution of $a(D)=a(\partial / i \partial x)$ with support in $K=K(A, \vartheta)$. Suppose that $x \bar{\epsilon} W(A, \vartheta), x \bar{\epsilon}-K$. Then $E(a, x)$ is holomorphic and

$$
D^{\nu} E(a, x)=i(2 \pi)^{1-n} \int_{\alpha^{*}} \chi_{q}^{0}(i x \xi) \xi^{\nu} a(\xi)^{-1} \omega(\xi)
$$

when $q=m-n-|\nu| \geqslant 0$ and

$$
D^{\nu} E(a, x)=(2 \pi)^{-n} \int_{t_{x} \partial \alpha^{*}} \chi_{q}^{0}(i x \xi) \xi^{\nu} a(\xi)^{-1} \omega(\xi)
$$

when $q<0$. Here 


$$
\chi_{r}^{0}(t)=t^{r} / r !, r \geqslant 0, \quad \chi_{r}^{0}(t)=(-1)^{r+1}(-r-1) ! t^{r}, r<0
$$

and

$$
\alpha^{*}=\alpha(A, x, \vartheta)^{*} \in H_{n-1}\left(Z^{*}-A^{*}, X^{*}\right)
$$

is the homology class of Definition 6.14.

Note. The differential $(n-1)$-forms $\varphi$ and $\varphi^{\prime}$ appearing in (7.17) and $\left(7.17^{\prime}\right)$ are rational homogeneous of degree zero and induce rational $(n-1)$-forms on the projective space $Z^{*}$ (see Part II). By (7.4), they are closed and it is clear that $\varphi$ is holomorphic on $Z-A$ and vanishes on $X$, while $\varphi^{\prime}$ is holomorphic on $Z-A \cup X$. Hence, if $\alpha, \alpha^{\prime}$ are $(n-1)$-cycles of $(Z-A, X)$ and $Z-A \cup X$ respectively, then $\int_{\alpha} \varphi$ and $\int_{\alpha^{\prime}} \varphi^{\prime}$ depend only on the homology classes $\alpha^{*}$ and $\alpha^{\prime *}$ of $\alpha$ and $\alpha^{\prime}$ respectively. In particular, if $\alpha$ or $\alpha^{\prime}$ is homologous to zero, the corresponding integral vanishes. Hence (6.21) combined with (7.17), (7.17') reflects the basic fact that $E(x)=0$ outside $K(A, \vartheta)$. As remarked in the note following Theorem 7.5 , our formulas are interesting only when $a$ is a complete polynomial.

This theorem has the following corollary, which is important in the theory of lacunas to be exposed in the next section. Let $p$ be an integer. A function $Q(x)$ defined in some open conical set $M$ is said to have homogeneity $p$ if $Q(\lambda x)=\lambda^{p} Q(x)$ for $x \in M$ and $\lambda>0$. It is obvious that polynomials of negative homogeneity vanish.

\subsection{Corollary. If, for some $x$,}

$$
\partial \alpha(A, x, \vartheta)^{*}=0 \quad \text { in } H_{n-2}\left(X^{*}-X^{*} \cap A^{*}\right),
$$

and $b \in \mathrm{Hyp}(\vartheta, m k)$ is sufficiently close to $a^{k}, k=1,2, \ldots$, then $E(b, \cdot)$ is a polynomial of homogeneity $m k-n$ in the component of the complement of $W(B, \vartheta)$ that contains $x$.

7.21. Examples. When $n=2,(7.20)$ is always true so that $E(a, \vartheta, \cdot)$ is locally a polynomial outside $W(A, \vartheta)$ for every $a \in \mathrm{Hyp}(\vartheta)$. This is of course also immediate by elementary calculation. Let us choose coordinates such that $\vartheta=(1,0)$ and normalize $a \in \mathrm{Hyp}(\vartheta, m)$ such that $a(\vartheta)=i^{m}$. Then $a(\xi)$ is the product of $m$ purely imaginary factors $a_{k}(\xi)=i\left(\xi_{1}+\right.$ $\left.\lambda_{k} \xi_{2}\right)$ and we have $E_{k}(x)=E_{k}\left(a_{k}, \vartheta, x\right)=H\left(x_{1}+\lambda_{k} x_{2}\right) \delta\left(-\lambda_{k} x_{1}+x_{2}\right)$ where $H$ is the Heaviside function, $H(t)=1$ when $t \geqslant 0$ and 0 otherwise. Further, $E(a, x)=\left(E_{1} * \ldots\right)(x)$ is a convolution. The wave front surface $W=W(A, \vartheta)$ consists of all half-rays $\left(\varrho, \varrho \lambda_{k}\right)$ with $\varrho \geqslant 0, K=$ $K(A, \vartheta)$ is their convex hull and, if $a$ is complete, $m \geqslant 2$ then $E(a, \cdot)$ is a positive polynomial of homogeneity $m-2$, in each component of $K-W$. In particular $\mathrm{S} E(a, \cdot)=K$, a fact which will be of some importance later. By (6.22), if $n$ is even and $\operatorname{Re} \dot{X} \cap A$ is empty, $E(a, \vartheta, \cdot)$ is a polynomial in the component $L$ of the complement of $W$ that contains $x$. This applies e.g. to a product of wave operators with different speeds of light and $L$ equal 
to the smallest dual light-cone. Any $a \in \mathrm{Hyp}(\vartheta)$ close to such a product also has the corresponding property.

Proof of the corollary. Since all positive powers of $a$ have the same associated hypersurface $A$, it suffices to consider the case $k=1$. If (7.20) holds, then $t_{x} \partial \alpha^{*}=0$ in $H_{n-1}\left(Z^{*}-A^{*} \cup X^{*}\right)$ so that, by $\left(7,17^{\prime}\right)$, all derivatives of order $>m-n$ of $E(a, \cdot)$ vanish at $x$. Now, by Lemma 6.23, (7.20) implies the same equality with $x, A$ replaced by $y, B$ provided $y$ is close to $x$ and $b \in \operatorname{Hyp}(\vartheta, m)$ is close to $a$. Hence, for every such $b, E(b, \cdot)$ is a polynomial of degree at most $m-n$ close to $x$. But then, by analytical continuation, $E(b, ;)$ is such a polynomial in the entire component $L(x, B)$ of the complement of $W(B, \vartheta)$ that contains $x$. Since $E(b, \cdot)$ is homogeneous of degree $m-n$, the polynomial has homogeneity $m-n$. This finishes the proof.

Proof of the theorem. Since $x \bar{\epsilon} W(A, \vartheta), x \bar{\epsilon}-K$, we have $x \bar{\epsilon} \pm W(A, \vartheta)$ so that Theorem $7.5 \mathrm{~d})$ applies. Letting $v \in V(A, X, \vartheta)$ and $s=1$ and using (1.8), differentiating (7.7) $\nu$ times gives

$$
\begin{aligned}
D^{\nu} E(x)= & (2 \pi)^{-n} i^{a} \int_{\gamma=1} \chi_{q}(x \xi) \zeta^{\nu} a(\zeta)^{-1} \omega(\zeta) \\
& -(2 \pi)^{-n} \int_{\gamma=1} \chi_{q}^{0}(i x \xi) \zeta^{\nu} a(\zeta)^{-1}\left(2^{-1} \pi i-m^{-1} \log a(\zeta)\right) \omega(\zeta),
\end{aligned}
$$

where $\zeta=\xi-i v(\xi)$. Now since $x \bar{\epsilon}-K$ and $E$ vanishes outside $K$, the right side vanishes if $x$ is replaced by $-x$. Hence

Now, by (1.16)

$$
D^{\nu} E(x)=D^{v} E(x)-(-1)^{\natural} D^{\nu} E(-x)
$$

$$
\chi_{a}(x \xi)-(-1)^{q} \chi_{a}(-x \xi)=2 \pi i \sigma_{q}(x \xi)
$$

while, if $\chi$ is replaced by $\chi^{0}$, the corresponding expression vanishes. This gives

$$
D^{\nu} E(x)=i(2 \pi)^{1-n} \int_{\gamma=1} i^{a} \sigma_{a}(x \xi) \zeta^{\nu} a(\zeta)^{-1} \omega(\zeta)
$$

If $q \geqslant 0$, then, by (1.17),

$$
\sigma_{q}(x \xi)=2^{-1} \operatorname{sgn}(x \xi) \chi_{\varepsilon}^{0}(x \xi)
$$

so that

$$
D^{\nu} E(x)=i(2 \pi)^{1-n} \int_{\gamma=1} 2^{-1} \operatorname{sgn}(x \xi) \chi_{a}^{0}(i x \zeta) \zeta^{\nu} a(\zeta)^{-1} \omega(\zeta) .
$$

where $\zeta=\xi-i v(\xi)$. In view of the definition 6.14 of $\alpha^{*}$, this is precisely (7.17) with $\zeta$ as the integration variable. Next, let $q<0$. Choose coordinates such that $x=(1,0, \ldots, 0)$. Since 12-702909 Acta mathematica. 124. Imprimé le 14 Avril 1970. 


$$
\omega(\xi)=-d \xi_{1} \wedge \omega\left(\xi_{2}, \ldots, \xi_{n}\right)+\xi_{1} d \xi_{2} \wedge \ldots \wedge d \xi_{n},
$$

this choice gives $\omega_{x}(\xi)=-\omega\left(\xi_{2}, \ldots, \xi_{n}\right)$, (see $(6.10)$ ). Let us also make $\gamma\left(\xi_{1}, \ldots, \xi_{n}\right)$ independent of $\xi_{1}$ when $\xi_{1}$ is small compared to $\gamma(\xi)$. Then, since $x v(\xi)=v_{1}(\xi)=0$, we have

$$
\omega(\zeta)=d \zeta_{1} \wedge \omega_{x}\left(\zeta_{2}, \ldots, \zeta_{n}\right), \quad \zeta=\xi-i v(\xi)
$$

on $\gamma(\xi)=1$. Since, by $(1.7), \sigma_{q}(x \xi)=\delta^{(-q-1)}(x)$ when $q<0$, (7.22) gives

$$
D^{\nu} E(x)=i(2 \pi)^{1-n} i^{a} \int_{\partial \alpha^{\prime}}\left(-\partial / \partial \zeta_{1}\right)^{-a-1} \zeta^{\nu} a(\zeta)^{-1} \omega_{x}\left(\zeta_{2}, \ldots, \zeta_{n}\right),
$$

where

$$
\partial \alpha^{\prime}=\{\zeta ; \zeta=\xi-i v(\xi), \xi \in \operatorname{Re} X \cap\{\gamma(\xi)=1\}\}
$$

is an $(n-2)$-cycle on $X-X \cap A$ oriented by $\omega_{x}(\xi)>0$. Hence, if $r>0$ is small, by the definition (7.18) of $\chi_{q}^{0}(t)$ and Cauchy's formula in one variable, we have

$$
D^{\nu} E(x)=(2 \pi)^{-n} \int \chi_{q}(i x \zeta) \zeta^{\nu} a(\zeta)^{-1} d \zeta_{1} \wedge \omega_{x}\left(\zeta_{x}, \ldots, \zeta_{n}\right)
$$

with $\omega\left(\operatorname{Re} \zeta_{1}, \operatorname{Im} \zeta_{1}\right) \wedge \omega_{x}\left(\zeta_{2}, \ldots, \zeta_{n}\right)>0$ on the product $\beta=\left\{\left|\zeta_{1}\right|=r\right\} \times \partial \alpha^{\prime}$. Here, by the definition of $\alpha^{*}$ and $t_{x}, \beta$ represents $t_{x} \partial \alpha^{*}$. This proves (7.17').

Note. That fundamental solutions of homogeneous elliptic operators can be expressed in terms of 'algebraic' integrals was discovered by Fredholm (1900) whose work was extended to hyperbolic operators by Herglotz (1926-28) and Petrovsky (1945). Petrovsky's formulas (l.c. p. 315 and 324) result from (7.17) and (7.17') when $\nu=0$ by taking one residue onto $\left(A^{*}, X^{*}\right)$ and two successive residues onto $A^{*} \cap X^{*}$ respectively when $a$ is strongly hyperbolic and $A^{*}$ is regular. Petrovsky's proofs are rather complicated but have later been simplified by Leray (1952). Leray has also extended the formalism involved to a general Laplace transform that gives explicit formulas for fundamental solutions of certain strongly hyperbolic operators with analytic coefficients (Leray 1962). In the cases that he considers, Leray employs the homology class $\alpha^{*}$.

It is also possible to express $E(x)=E(a, \vartheta, x)$ in terms of the distributions

$$
a_{ \pm}(\xi)^{-1}=\lim a(\xi \pm i s \vartheta)^{-1}, \quad 0<s \rightarrow 0 .
$$

Taking $0 \leqslant \gamma(\xi) \in C(\operatorname{Re} \dot{Z})$ such that $\gamma(\lambda \xi)=|\lambda| \gamma(\xi)$ when $\lambda \in R$ and $\gamma(\xi)=0$ if and only if $\xi$ is proportional to a fixed $\eta \in \Gamma(A, \vartheta)$, the following formula can be deduced from (4.11),

$$
E(x)=(2 \pi)^{-n} i^{m-n} \int \chi_{m-n}(x \xi) a_{-}(\xi)^{-1} \delta(\gamma(\xi)-1) d \xi .
$$


It is true for all $x$ if the product of distributions appearing on the right is interpreted properly. Using the fact that $E(x)=0$ outside $K=K(A, \vartheta)$, this gives

$$
E(x)=2^{-1} i(2 \pi)^{1-n} i^{m-n} \int \sigma_{m-n}(x \xi) a_{-}(\xi)^{-1} \delta(\gamma(\xi)-1) d \xi
$$

when $x \bar{\epsilon}-K$. We can also use the fact that $E(a,-\vartheta, x)$ vanishes outside $-K$ and get

$$
E(x)=(2 \pi)^{-n} i^{m-n} \int \chi_{m-n}(x \xi)\left(a_{-}(\xi)^{-1}-a_{+}(\xi)^{-1}\right) \delta(\gamma(\xi)-1) d \xi
$$

when $x \bar{\epsilon}-K$. Here $i^{-m} E(x)$ is real and $a_{-}(\xi)^{-1}-a_{+}(\xi)^{-1}$ is purely imaginary and, separating real and imaginary parts, we get the formulas for $E(x)$ which, for strongly hyperbolic $a$ have been employed by Gelfand and Shilov (1958).

The inhomogeneous case. Let $P \in$ hyp $(\vartheta, m)$, let $a \in \operatorname{Hyp}(\vartheta, m)$ be the principal part of $P=a+b$ and let $E=E(P, \vartheta, x)$ be the fundamental solution of $P$ with support in $K(P, \vartheta)=$ $K(A, \vartheta)$, so that, by Theorem 4.1,

$$
E=\sum_{0}^{\infty}(-1)^{k} b(D)^{k} E\left(a^{k+1}, \vartheta, x\right)
$$

where the series converges in the distribution sense.

7.24. ТнЕовем. Outside $W(A, \vartheta)$, the series (7.23) converges locally uniformly and $E(P, \vartheta, x)$ is a holomorphic function there. If every $E\left(a^{k+1}, \vartheta, x\right)$ is a polynomial in some component of the complement of $W(A, \vartheta), E(P, \vartheta, x)$ is an entire function there.

Proof. Since $E$ vanishes outside $K=K(A, \vartheta)$, it suffices to investigate (7.23) when $x \in K-W(A, \vartheta)$. Let $c(\xi)$ be a homogeneous polynomial of degree $j$. We can use (7.7) to compute $c(D) E\left(a^{k+1}, \vartheta, x\right)$ simply by replacing $a$ by $a^{k+1}$ and carrying out $c(D)$ under the sign of integration, using (1.8). This gives

$$
\begin{aligned}
c(D) E\left(a^{k+1}, \vartheta, x\right)= & (2 \pi)^{-n} \int_{\gamma=1} i^{q} \chi_{q}(x \zeta) c(\zeta) a(\zeta)^{-k-1} \omega(\zeta) \\
& -(2 \pi)^{-n} \int_{\gamma=1} \chi_{q}^{0}(i x \zeta) c(\zeta) a(\zeta)^{-k-1}\left(2^{-1} \pi i-m^{-1} \log a(\zeta)\right) \omega(\zeta)
\end{aligned}
$$

where $q=m(k+1)-n-j, \zeta=\xi-i v(\xi)$. Let $N$ be a complex neighbourhood of $x$ which is so small that $|y \zeta| \neq 0$ when $y \in N$. Put

$$
t_{a}=\min |a(\zeta)|, \quad t_{c}=\max |c(\zeta)|, \quad t_{0}=(1+\max |y \zeta|)
$$

when $\gamma(\xi)=1, y \in N$. Then (1.11) and (1.12) and obvious estimates show that 


$$
\left|c(D) E\left(a^{k+1}, \vartheta, y\right)\right| \leqslant C t_{0}^{q} t_{a}^{-k-1} t_{c} \Gamma(q-1)^{-1},
$$

where $y \in N, q>1$ and $C$ depends only on $m, n$. Next, put

$$
b(\xi)=b_{0}(\xi)+\ldots+b_{m-1}(\xi),
$$

where $b_{j}(\xi)$ is homogeneous of degree $j$. Developing we have

$$
b(D)^{k}=\sum\left(\begin{array}{c}
k \\
v
\end{array}\right) b_{0}(D)^{\varphi_{1}} \ldots b_{m-1}(D)^{p_{m-1}}
$$

where $\nu=\left(\nu_{0}, \ldots, v_{m-1}\right)$ has integral components and $v_{0}+\ldots+v_{m-1}=k,\left(\begin{array}{l}k \\ v\end{array}\right)$ are the multinomial coefficients and the degree of

$$
c(D)=b_{0}(D)^{\nu_{\bullet}} \ldots b_{m-1}(D)^{\nu_{m-1}}
$$

is $j=0 v_{0}+1 v_{1}+\ldots+(m-1) v_{m-1} \leqslant k(m-1)$ and $t_{c} \leqslant C_{1}^{k}$ for some $C_{1}$. Hence, by (7.25)

$$
\left|b(D)^{k} E\left(a^{k+1}, \vartheta, y\right)\right| \leqslant C \sum\left(\begin{array}{l}
k \\
\nu
\end{array}\right) t_{0}^{(m+1) k} t_{a}^{-k-1} C_{1}^{k} \Gamma(p-1)^{-1}
$$

when $p=\min (m(k+1)-j-n)=k+m-n>0$, which is true for $k$ sufficiently large. Summing over the multinomial coefficients gives a new majorant

$$
\left|b(D)^{k} E\left(a^{k+1}, \vartheta, y\right)\right| \leqslant C C_{2}{ }^{k} \Gamma(k+m-n-1)^{-1},
$$

where $y \in N$ and $C_{2}$ is independent of $k$. Hence the series (7.23) converges uniformly in $N$ and this proves the first part of the theorem. To prove the second part note that if $c(D)=D^{\mu}$ with $|\mu|=m(k+1)-n$ then the arguments that lead to (7.25) show that

$$
\left|D^{\mu} \mathbb{E}\left(a^{k+1}, \vartheta, x\right)\right| \leqslant C_{3}^{k+1},
$$

where $C_{3}$ is a constant independent of $\mu$. If $E\left(a^{k+1}, \vartheta, \cdot\right)$ is a polynomial $Q_{k+1}(y)$ in the component $L(x, A)$ of the complement of $W(A, \vartheta)$ that contains $x$, then this polynomial has homogeneity $q_{k}=m(k+1)-n$ and hence, by (7.26),

$$
Q_{k+1}(y)<<C_{3}^{k+1}\left(y_{1}+\ldots+y_{n}\right)^{q_{k}} / \Gamma\left(q_{k}+1\right)
$$

in the sense of dominating power series. As before, this gives

$$
b(D)^{k} Q_{k+1}(y)<<C_{4}^{k}\left(y_{1}+\ldots y_{n}\right)^{k} / \Gamma(k+m-n+1)
$$

with a new constant $C_{4}$. Hence $E(P, \vartheta, \cdot)$ equals an entire function in $L(x, A)$ and this finishes the proof. 


\section{Chapter 3. Sharp fronts and lacunas}

We shall review some basic notions and results of the theory of lacunas and make the connection with Petrovsky's work. For Petrovsky, a lacuna for an operator $P \in$ hyp ( $\vartheta$ was a component of the complement of the wave front surface $W(P, \vartheta)$ where the fundamental solution $E(P, \vartheta, \cdot)$ vanishes. We shall take a wider point of view suggested by $\mathrm{L}$. Hörmander and based on the notion of sharp wave front. Most of the results that we state use topological properties of algebraic manifolds that will be proved in Part II.

\section{Supports and singular supports of fundamental solutions}

Knowing that the fundamental solution $E(P)=E(P, \vartheta, \cdot)$ of a hyperbolic operator $P \in$ hyp $(\vartheta)$ vanishes outside $K(P, \vartheta)$ and is holomorphic outside the wave front surface $W(P, \vartheta)$ gives some but not all information about the support $\mathrm{S} E(P)$ and the singular support $\operatorname{SS} E(P)$ of $E(P)$. We know only that

$$
\begin{gathered}
\mathrm{S} E(P) \subset K(P, \vartheta) \\
\mathrm{SS} E(P) \subset W(P, \vartheta) .
\end{gathered}
$$

As we shall see in a moment, the two inclusions may be proper. We begin by stating some simple cases when there is equality. The following lemma employs the notations above, $a \in \operatorname{Hyp}(\vartheta)$ is the principal part of $P \in$ hyp $(\vartheta), L(A)=L(P)$ their common lineality and $n(A)=n(P)$ the reduced dimension, i.e. the codimension of $L(A)$. An index $\xi$ indicates the corresponding objects for the localizations $P_{\xi}, a_{\xi}$.

8.3. Lемм. If the reduced dimension $n(P)$ of $P$ is $\leqslant 2$, in particular if $n \leqslant 2$, then

$$
\mathrm{S} E(P)=K(A, \vartheta) \text {. }
$$

If the reduced dimension $n_{\xi}(P)$ of $P_{\xi}$ is $\leqslant 2$ for all $0 \neq \xi \in \operatorname{Re} Z$, in particular if $\operatorname{Re} \dot{A}$ is regular or if $n \leqslant 3$, then

$$
\operatorname{SS} E(P)=W(A, \vartheta)
$$

Proof. If $n(P)=0, P=a$ is a non-vanishing constant and the statements are trivial. Next, we shall see that it suffices to prove (8.4) for complete polynomials. In fact, choose coordinates $x=\left(x^{\prime}, x^{\prime \prime}\right)$ and dual coordinates $\xi=\left(\xi^{\prime}, \xi^{\prime \prime}\right)$ such that $\xi^{\prime}=0$ characterizes $L(A)$. Then $P^{\prime}\left(\xi^{\prime}\right)=P^{\prime}(\xi)$ and $a^{\prime}\left(\xi^{\prime}\right)=a(\xi)$ are complete polynomials in $\xi^{\prime}$ and $E(P, \vartheta, x)=$ $E\left(P^{\prime}, \vartheta^{\prime}, x\right) \delta\left(x^{\prime \prime}\right)$ so that $x \in \mathbb{S} E(P)$ if and only if $x^{\prime} \in \mathbb{S} E\left(P^{\prime}\right)$ and $x^{\prime \prime}=0$. On the other hand, $x \in K(A, \vartheta)$ if and only if $x^{\prime} \in K\left(A^{\prime}, \vartheta^{\prime}\right)$ and $x^{\prime \prime}=0$. Hence (8.4) holds for $P$ if and only if it 
holds for $P^{\prime}$. The proof of (8.4) when $n=1$ is immediate and is left to the reader. Also when $n=2$ and $P$ is complete, the proof is elementary. In fact, letting $a$ be the principal part of $P$, we know from the examples 7.21 that $\mathrm{S} E(a)=K$. Now, by Theorem 7.24, $E(P, \vartheta, \cdot)$ is holomorphic outside $W$ and hence, if $\mathrm{S} E(P) \neq K, E(P)$ has to vanish in some component of $K-W$. But then, by (4.7), $E(a)$ also vanishes there which is impossible. Hence (8.4) follows. Finally, (8.5) is a consequence of (8.4) and the Localization Theorem 4.10 that says that $\operatorname{SS} E(P)$ contains all $\mathrm{S} E\left(P_{\xi}\right)$ for $\xi \in \operatorname{Re} \dot{Z}$. In fact, if $n_{\xi}(P) \leqslant 2$, then by (8.4) $\mathrm{S} E\left(P_{\xi}\right)=$ $K\left(A_{\xi}, \vartheta\right)$. If this is true for all $0 \neq \xi \in \operatorname{Re} Z$, by the definition of $W(A, \vartheta)$, we have (8.5).

We now pass to some examples showing that (8.1) or both (8.1) and (8.2) may be proper inclusions.

8.6. Examples. When $a=\Delta^{p}$ is a power of the wave operator, $a \in \mathrm{Hyp}(\vartheta)$ with $\vartheta=$ $(1,0, \ldots, 0), K=K(A, \vartheta)=K(\Delta, \vartheta)$ is the future light-cone $x_{1} \geqslant 0, x_{1}^{2}-x_{2}^{2}-\ldots-x_{n}^{2} \geqslant 0$ and $W=W(A, \vartheta)=W(\Delta, \vartheta)$ its boundary. Since $\operatorname{Re} \dot{A}: \xi_{1}^{2}-\xi_{2}^{2}-\ldots-\xi_{n}^{2}=0, \xi \neq 0$, is regular, we know from Lemma 8.3 that $\operatorname{SS} E(a)=W$, and this also follows from (4.20). However, by (4.21), if $2 p<n$ and $n$ is even, $E(a)$ vanishes in $\stackrel{\circ}{K}$ so that $\mathrm{S} E(a)=W \neq K$. The simplest case when this happens is when $p=1, n=4$ which corresponds to the propagation of light in free space-time. That $\mathrm{S} E(a)=W$ reflects the possibility of omitting sharp light-signals and is sometimes referred to as Huygens' principle. If $P \in$ hyp $(\vartheta)$ has principal part $a=\Delta^{p}$ and $P \neq \Delta^{p}$, it is probable that $\mathrm{S} E(P)=K$, but if, e.g., $n=6$ there are operators $P=\Delta$ plus lower terms with non-constant coefficients such that $\mathrm{S} E(P)=W$ (Stellmacher (1955)).

8.7. It is easy to construct examples where both (8.1) and (8.2) or only (8.2) are proper inclusions. In fact, let $a \in \operatorname{Hyp}(\vartheta)$ be a product $a^{\prime}\left(\xi^{\prime}\right) a^{\prime \prime}\left(\xi^{\prime \prime}\right)$ where $\xi=\left(\xi^{\prime}, \xi^{\prime \prime}\right)$ is a partition of the coordinates and put $E(a)=E(a, \vartheta, x), E\left(a^{\prime}\right)=E\left(a^{\prime}, \vartheta^{\prime}, x^{\prime}\right), E\left(a^{\prime \prime}\right)=E\left(a^{\prime \prime}, \vartheta^{\prime \prime}, x^{\prime \prime}\right)$ and $K=K(A, \vartheta), W=W(A, \vartheta)$ etc. Then $\mathrm{S} E(a)=\mathrm{S} E\left(a^{\prime}\right) \times \mathrm{S} E\left(a^{\prime \prime}\right), K=K^{\prime} \times K^{\prime \prime}$ and

$$
\begin{gathered}
\operatorname{SS} E(a)=\left(\operatorname{SS} E\left(a^{\prime}\right) \times \operatorname{SE} E\left(a^{\prime \prime}\right)\right) \cup\left(\operatorname{S} E\left(a^{\prime}\right) \times \operatorname{SS} E\left(a^{\prime \prime}\right)\right) \\
W=\left(W^{\prime} \times K^{\prime \prime}\right) \cup\left(K^{\prime} \times W^{\prime \prime}\right) .
\end{gathered}
$$

In fact, $W$ is the union of all $K_{\xi}=K_{\xi^{\prime}}^{\prime} \times K_{\xi^{\prime \prime}}^{\prime \prime}$ for $\xi \neq 0$, i.e. for $\xi^{\prime} \neq 0$ or $\xi^{\prime \prime} \neq 0$. Hence, if, e.g., $\mathrm{S} E\left(a^{\prime}\right)=\mathrm{SS} E\left(a^{\prime}\right)=W^{\prime} \neq K^{\prime}$ we get proper inclusions in (8.1) and also in (8.2). In fact, then $\mathrm{S} E(a)=W^{\prime} \times \mathrm{S} E\left(a^{\prime \prime}\right) \subset W^{\prime} \times K^{\prime \prime}$ is a proper part of $K$ and $\operatorname{SS} E(a) \subset$ $\left(W^{\prime} \times K^{\prime \prime}\right) \cup\left(W^{\prime} \times W^{\prime \prime}\right)$ does not contain $\left(K^{\prime}-W^{\prime}\right) \times W^{\prime \prime} \subset W$. Note that these circumstances prevail when $a^{\prime \prime}$ is replaced by anyone of its powers. Hence, for given $n$, both (8.1) and (8.2) may be proper inclusions when $P=a$ has arbitrarily large homogeneity. The simplest choice we can make is to put $a^{\prime}\left(\xi^{\prime}\right)=\xi_{1}^{2}-\ldots-\xi_{q}^{2}, a^{\prime \prime}\left(\xi^{\prime \prime}\right)=\xi_{n}^{p}$ where $4 \leqslant q<n$ is even and $p \geqslant 0$ is arbitrary. However, note that by replacing $a^{\prime}$ by $a^{\prime k}$ with $2 k \geqslant q$, we have $\mathrm{S} E\left(a^{\prime k}\right)=K^{\prime}$ $\operatorname{SSE}\left(a^{\prime k}\right)=W^{\prime}$ so that $(8.1),(8.2)$ hold with equality for high powers of $a$. 
Next we shall give an example where (8.1) holds with equality but (8.2) is a proper inclusion. Let $a_{1}(\xi)=\xi_{1}^{2}-\ldots \xi_{q}^{2}$ with $q$ as before and put $a_{2}(\xi)=2^{-1} \xi_{1}^{2}-\ldots \xi_{n}^{2}$. Then $a=a_{1} a_{2}$ is a complete polynomial in Hyp $(\vartheta), \vartheta=(1,0, \ldots, 0)$. Here $K_{1}=K\left(A_{1}, \vartheta\right) \subset K_{2}=K\left(A_{2}, \vartheta\right)$ and $K_{2}=K=K(A, \vartheta)$ is the cone $x_{1} \geqslant 0,2 x_{1}^{2} \geqslant x_{2}^{2}+\ldots+x_{n}^{2}$. Further, $W=W(A, \vartheta)$ is the union of $W_{1}=W\left(A_{1}, \vartheta\right)=K_{1}$ and $W_{2}=W\left(A_{2}, \vartheta\right)=\partial K_{2}$. Note that $\operatorname{dim} W_{1}=q<n=\operatorname{dim} W_{2}$ and that $W_{1}$ and $W_{2}$ intersect only at the origin. An explicit calculation of $E(a)=E\left(a_{1}\right) * E\left(a_{2}\right)$ shows that $\mathrm{S} E(a)=K$ while $\operatorname{SS} E(a)$ consists of $W_{2}$ and $\partial K_{1} \neq W_{1}$. In this case, (8.1) holds with equality but (8.2) is a proper inclusion. Again, taking $2 k \geqslant q$ we have $\mathrm{S} E\left(a_{1}^{p}\right)=K_{1}$ and an explicit calculation (or an appeal to the Localization Theorem 4.10) shows that $\operatorname{SS} E\left(a^{k}\right)=W$.

8.8. Example. (Gårding (1947).) Let $\operatorname{Re} Z^{\prime}$ be real $n^{2}$-space represented by $n \times n$ hermitian matrices $x=\left(x_{j k}\right)$ and let $\operatorname{Re} Z$ be another copy of this space put in duality with $\operatorname{Re} Z$ by the bilinear form $\langle\xi, x\rangle=\operatorname{tr} \xi x$. Interpret $\partial / \partial x_{j k}, j \neq k$, in the usual way for a complex variable so that $\left(\partial / \partial x_{j k}\right)\langle\xi, x\rangle=\xi_{j k}$. Put $a(\xi)=\operatorname{det} \xi$ and let $\Gamma$ be the cone of all positive definite matrices $\vartheta \in \operatorname{Re} Z$. Then $a \in \operatorname{Hyp}(\vartheta, n)$ for all $\vartheta \in \Gamma$ and $\Gamma(A, \vartheta)=\Gamma$. The dual cone $K=K(A, \vartheta)$ characterized by $\operatorname{tr} x \Gamma \geqslant 0$ consists of all $x \geqslant 0$ and the wave front surface $W=W(A, \vartheta)$ is the boundary of $K$. Let $E_{p}(x)$ be the fundamental solution of $a(\partial / \partial x)^{p}$ with support in $K$. In the paper quoted it is shown that $\mathrm{S}\left(E_{p}\right)$ consists of all $x \in K$ which are of rank $\leqslant p$. Also, if $k \geqslant n, E\left(a^{k}, \vartheta, x\right)$ is a nonvanishing multiple of $(\operatorname{det} x)^{k-n}$. Hence $\mathrm{S}\left(E_{p}\right)=K$ if and only if $p \geqslant n$, while, if $p=n-1, \mathrm{~S}\left(E_{p}\right)=\operatorname{SS}\left(E_{p}\right)=W$ and, if $p<n-1$, $\mathrm{S}\left(E_{p}\right)=\mathrm{SS}\left(E_{p}\right) \neq W$. If, e.g., $p=1$, then $\mathrm{S}\left(E_{1}\right)=\mathrm{SS}\left(E_{1}\right)$ has dimension $2 n-1$ which should be compared to the dimension $n^{2}-1$ of $W$. Also, when $p<n-1$, the complement of $\mathrm{S}\left(E_{p}\right)=$ $\mathrm{SS}\left(E_{p}\right)$ is connected. Similar examples have been found by Gindikin and Vajnberg (1967) and for certain parabolic operators by Gindikin (1967).

From the examples above one gets the impression that raising an operator $P$ to a high enough power will give equality in (8.1), (8.2). This is indeed the case.

The following theorem will be proved in Part II.

8.9. ТhEOrem. Let $P \in \operatorname{hyp}(\vartheta, m)$ and let $a \in \operatorname{Hyp}(\vartheta, m)$ be its principal part. There are integers $k_{0}$ and $k_{1}$ depending only on $m$ and the reduced dimension $n(P)$, such that

$$
\begin{aligned}
\operatorname{SS} E\left(P^{k}\right) & =W(A, \vartheta) \\
\operatorname{SE}\left(P^{k}\right) & =K(A, \vartheta)
\end{aligned}
$$

when $k \geqslant k_{0}$ and $k \geqslant k_{1}$ respectively.

Note that (8.10) is a corollary of (8.11) and that, choosing $k_{1}$ as a monotone function of $m$ and $n=n(P)$, we have $k_{0}(m, n) \leqslant k_{1}(m-1, n-1)$. In fact, by the Localization The- 
orem 10.4, we have $\operatorname{SSE}\left(P^{k}\right) \supset \operatorname{SE} E\left(P_{\xi}^{k}\right)$ for all $\xi \in \operatorname{Re} \dot{Z}$ and $m\left(P_{\xi}\right)<m, n\left(P_{\xi}\right)<n$ so that, by (8.11), $\operatorname{SE}\left(P_{\xi}^{k}\right)=K(A, \vartheta)$ for all $\xi$ and consequently also $\operatorname{SS} E\left(P^{k}\right) \supset W(A, \vartheta)$ when $k \geqslant$ $k_{1}(m-1, n-1)$. More precise estimates are also possible. If $\operatorname{Re} A$ is sufficiently regular or if certain stability requirements are imposed, (8.10) holds for $k \geqslant 1$ and (8.11) when $m k-n \geqslant 0$.

\section{Sharp fronts, lacunas, regular lacunas}

Petrovsky (1945), defined lacunas for a hyperbolic operator $P$ with fundamental solution $E$ as components of the complement of the wave front surface $W$ in which $E$ vanishes. The outside of the cone of propagation $K$ is a lacuna, called the trivial lacuna. The lacunas inside $K$ are of course important when we want to study the support of $E$, but as a rule, the property that $E$ vanishes is not stable under addition of lower terms or a passage to variable coefficients. Following a suggestion by L. Hörmander, we shall define lacunas by a weaker property.

Starting with a general situation, let $u$ be a distribution defined in an open subset $O$ of $R^{n}$. We may think of $u$ as describing the movement in space-time of a general elastic $(n-1)$-dimensional medium. Let $C(u)$ be the maximal open subset of $O$ where $u$ is a $C^{\infty}$ function. The complement of $C(u)$ is then the singular support $\operatorname{SS}(u)$ of $u$. The following definition is motivated in the introduction.

9.1. Definition. Let $L$ be a component of $C(u)$. The distribution $u$ is said to be sharp from $L$ at a point $y \in \partial L$ if $u$ has a $C^{\infty}$-extension from $L$ to $L \cap M$ for some neighbourhood $M$ of $y$. When $u$ is sharp at all points of $\partial L, \operatorname{then} L$ is said to be a lacuna for $u$. If $u$ vanishes in $L, L$ is said to be strong lacuna for $u$.

The requirements of this definition make sense for any open part $L$ of $O$ and it is sometimes convenient to allow $L$ to be smaller than a component of $C(u)$. We are going to deal with the case when $u=E=E(P, \vartheta, \cdot)$ is the fundamental solution of some $P \in$ hyp $(\vartheta)$. Here $E$ is holomorphic outside the wave front surface $W=W(P, \vartheta)$, but as we have seen, it may happen that the singular support of $E$ is a proper part of $W$, although this does not happen if we raise $P$ to a high enough power. In the sequel, components $L$ of $C W=\operatorname{Re} Z^{\prime}-W$ such that $E=E(P)$ is sharp from $L$ at all points of $\partial L$ are also called lacunas and, more specifically, regular lacunas. The word regular will be motivated later. When $L$ is a lacuna of $E$, we also say that it is a lacuna for $P$. The complement of $K(P, \vartheta)$ is a strong lacuna for $P$ called the trivial lacuna. When $\operatorname{SS}(E)=W$, in particular when $n \leqslant 3$, all lacunas are regular.

9.2. Examples. Let $P \in$ hyp $(\vartheta, m)$. When $n=1, E$ is sharp from both sides at $W=\{0\}$ and $W$ divides the real line into two lacunas one of which is the trivial one. The other lacuna 
is strong if and only if $m=0$. When $n=2$ and $P$ is complete, then, by virtue of Theorem 7.24, $P$ is an entire function outside $W$ so that the whole complement of $W$ consists of lacunas. Also, there are no strong lacunas apart from the trivial one. Consequently, if $n>2$ but $L(P)$ has codimension $\leqslant 2$, then $\mathrm{S} E=\mathrm{SS} E=W=K$ and there is only the trivial lacuna. When the principal part of $P$ is a power of the wave operator $\Delta^{p} \in \operatorname{Hyp}(\vartheta), \vartheta=(1,0, \ldots, 0)$, then $K: x_{1} \geqslant 0, x_{1}^{2}-x_{2}^{2}-\ldots-x_{n}^{2} \geqslant 0$ is the future light-cone and $W=\operatorname{SS} E$ its boundary. When $P=\Delta^{p}$, (4.21) shows that $E$ is nowhere sharp at $W$ from $\dot{K}$ if $n$ is odd and everywhere sharp at $W$ from $\stackrel{K}{K}$ if $n$ is even. Hence $\stackrel{\circ}{K}$ is a lacuna or not for $P$ according as $n$ is even or odd. By Theorem 4.1, this statement extends to the non-homogeneous case. Again, by (4.21), when $P=\Delta^{p}, \dot{K}$ is a strong lacuna if and only if $2 p<n$ and $n$ is even. Later we shall give examples of components $L$ of the complement of $W$ such that $E$ is $\operatorname{sharp}$ from $L$ at $\partial L$ except at the origin. Such an $L$ fails to be a lacuna. Irregular strong lacunas are to be found in the examples 8.7 and 8.8 .

There is a general criterion for sharpness that can be presented as follows. Let $n \geqslant 2$, $y \in W$ and assume that $W$ is smooth near $y$ and that its curvature has maximal rank $n-2$ there. If $f(x)=0$ is a real equation of $W$ near $y$ with grad $f(y) \neq 0$ and $f_{j}, f_{j k}$ are the components of grad $f(y)$ and $\operatorname{grad}^{2} f(y)$ respectively, this means that $y \neq 0$ and that the quadratic form $c=\sum f_{j k} z_{j} z_{k}$ restricted to the tangent plane $\sum f_{j} z_{j}=0$ has rank $n-2$. Let $v$ be the number of negative eigenvalues of $c$.

\subsection{THEOR EM. $E$ is sharp at $y$ from the side sgn $f=$ const if and only if}

$$
(\operatorname{sgn} f)^{n}=(-1)^{\nu}
$$

Note that (9.4) is independent of the choice of $f$. If $n$ is even, (9.4) shows that $E$ is sharp from both sides of $W$ if $v$ is even and non-sharp from both sides when $v$ is odd. If $n$ is odd, $E$ is sharp from one side but not from the other. We shall not prove Theorem 9.3 which is well known in somewhat less general forms (see Davidova (1945), Borovikov (1959), Gårding (1961), Leray (1962), Ludwig (1965)).

9.5. Examples. When $n=2, W$ consists of half-rays and (9.4) is identically true as it should be. When $n=3,(9.4)$ shows that $E$ is sharp or non-sharp at a curved piece of $W$ according to the following figure

$$
\text { non-sharp })^{W^{*}} \text { sharp }
$$

where $W^{*}$ is the image of $W$ in real projective space. As we shall see in the next section, when $n=3$, an $x$ in $K-W$ belongs to a lacuna if and only if $A^{*} \cap X^{*}$ is real. All the triangles in the figures $5 \mathrm{~b}$ have that property except those of the figure $5 \mathrm{~b} 8$ (dotted) where $\operatorname{Re} X^{*}$ 
must meet $\Gamma^{*}$ for $A^{*} \cap X^{*}$ to be real. However, the criterion applies to the dotted triangles of figure $5 \mathrm{~b} 8$ and hence $E$ is sharp from the corresponding conical regions except at the origin and the cusps (actually, $E$ is sharp at the cusps). There are other instances of the same phenomenon. Let $m, n$ both be even, let $P=a \in \mathrm{Hyp}^{0}(\vartheta)$ and assume that every component of $\operatorname{Re} \dot{A}$ bounds a convex cone. This would be true if, e.g., $a$ were the product of $m / 2$ wave operators with different speeds of light. Then $W$ consists of $m / 2$ telescoping pieces, each bounding a convex $n$-dimensional cone and $\mathbf{C} W$ has $1+(m / 2)$ components. One of them is the outer component $\mathbf{C} K$ and one is the inner component that bounds the smallest convex cone. Provided the curvature of $W$ has maximal rank outside the origin, (9.4) shows that $E$ is sharp from both sides of $W$ outside the origin. In fact, $v$ is either 0 or $n-2$. Further, $E$ is trivially sharp at the origin from $C K$ and, by the examples 7.21, also from the inner component of $\mathbf{G} W$. On the other hand, it has been shown by Borovikov (1961) that $E$ is not sharp at the origin from the other components of $C W$ at least when $a$ is irreducible.

The following lemma shows that sharpness at the origin is the essential feature of lacunas.

9.6. Lемма. Let $P \in$ hyp $(\vartheta)$ and let $a \in \mathrm{Hyp}(\vartheta)$ be the principal part of $P$. Let $L$ be $a$ component of $K(A, \vartheta)-W(A, \vartheta)$ and suppose that $E(P, \vartheta, \cdot)$ is sharp from $L$ at the origin. Then $L$ is a lacuna for a and $E(a, \vartheta, \cdot)$ is a polynomial in $L$. If $E\left(P_{k}, \vartheta, \cdot\right)$ is sharp from $L$ at the origin for a sequence of operators $P_{k} \in \mathrm{hyp}(\vartheta)$ with principal parts $a^{k}, k=1,2, \ldots$, then $L$ is a lacuna for any $Q \in \operatorname{hyp}(\vartheta)$ with principal part $a^{k}$.

Proof. Let $P \in$ hyp $(\vartheta, m)$. By Theorem 4.1, if $t \downarrow 0$, then

$$
t^{n-m}(\partial / \partial x)^{\nu} E(P, \vartheta, t x) \rightarrow(\partial / \partial x)^{\nu} E(a, \vartheta, x)
$$

in the distribution sense for every $v$. Now, since $E(P, \vartheta, \cdot)$ is sharp from $L$ at the origin, $(\partial / \partial t x)^{\nu} E(P, \vartheta, t x)$ tends to a limit as $t \downarrow 0$ for all $\nu$ when $x \in L$. It follows that $(\partial / \partial x)^{\nu} E(a, \vartheta, x)=0$ when $|\nu|>m-n$ and $x \in L$ so that $E(a, \vartheta, \cdot)$ is, in fact, a polynomial of homogeneity $m-n$ in $L$ and $L$ is a lacuna for $a$. The same reasoning applied to $P_{k}$ shows that $E\left(a^{k}, \vartheta, \cdot\right)$ is a polynomial in $L$. But then, by Theorem $7.24, E(P, \vartheta, \cdot)$ is an entire function in $L$. Replacing, in this argument, $a$ by a power of $a$ finishes the proof.

\section{Stability. Petrovsky lacunas}

10.1. Definition. Let $\mathcal{Y}$ be a subset of hyp $(\vartheta, m)$. A lacuna $L$ for $P \in \operatorname{hyp}(\vartheta, m)$ is said to be stable under $₹$ if every $Q \in \mathcal{F}$ close enough to $P$ has a lacuna $L_{Q}$ such that $L_{Q} \cap L$ tends to $L$ (i.e. absorbs any compact subset of $L$ ) as $Q$ tends to $P$. 
We shall also say that $L$ is stable under perturbations in $\mathcal{F}$. As a special case, $\mathcal{F}$ may be all of hyp $(\vartheta, m)$. Imposing stability leaves only the regular lacunas. In fact, we have

10.2. Lемма. A lacuna for $P \in$ hyp $(\vartheta, m)$, stable under all hyperbolic perturbations must be regular.

Proof. Let $0 \neq x \in W(P)=W(P, \vartheta)$. By Lemma 5.17 there are strongly hyperbolic operators $Q$ close to $P$ such that $W(Q)$ meets an arbitrarily small conical neighbourhood of $x$. Also, by Lemma 8.3, $W(Q)$ is the singular support of $E(Q, \vartheta, \cdot)$. Hence, if a lacuna $L$ for $P$ is stable under all hyperbolic perturbations, it cannot meet $W(P)$.

The Herglotz-Petrovsky-Leray formulas immediately give sufficient conditions for regular lacunas.

10.3. Th EOR м. Let $a \in \operatorname{Hyp}(\vartheta, m)$ be complete and suppose that

$$
\partial \alpha(A, x, \vartheta)^{*}=0 \text { in } H_{n-2}\left(X^{*}-A^{*} \cap X^{*}\right)
$$

for some $x \in K(A, \vartheta)-W(A, \vartheta)$. Then $x$ belongs to a regular lacuna $L$ for any $b \in \mathrm{Hyp}(\vartheta, \mathrm{km})$, $(k=1,2, \ldots)$ which is close enough to $a^{k}$ and $L$ is also a lacuna for any $P \in$ hyp $(\vartheta)$ whose principal part is such $a b$.

Proof. Corollary 7.19 and Theorem 7.24.

In a somewhat less general form, the condition (10.4) was invented by Petrovsky (1945). He considered only strongly hyperbolic operators $a$ with non-singular $A^{*}$. It is convenient to have a name for components $L$ of $K(A, \vartheta)-W(A, \vartheta)$ having the property (10.4) at all points. We shall call them Petrovsky lacunas for any $P \in$ hyp $(\vartheta)$ with principal part $a$. By Lemma 6.23, Petrovsky lacunas are stable under all hyperbolie perturbations.

Petrovsky also employed the stronger condition

$$
\alpha(A, x, \vartheta)^{*}=0 \quad \text { in } H_{n-1}\left(Z^{*}-A^{*}, X^{*}\right)
$$

which implies (10.4). According to (7.17) it also implies that $E(a, \vartheta, \cdot)$ vanishes close to $x$ so that $x$ would belong to a regular strong lacuna for $a$. However, this case is illusory since $(10.5)$ is not true when $x$ is inside $K(A, \vartheta)$. In fact, we shall prove in Part II that in this case, $\alpha(A, x, \vartheta)^{*}$ has a non-zero intersection number with $\Gamma(A, \vartheta)^{*} \in H_{n-1}\left(Z^{*}-X^{*}, A^{*}\right)$. Note that by (6.21), we know (10.5) to hold when $x$ is outside $\pm K(A, \vartheta)$. For this reason, the trivial lacuna will be included in the Petrovsky lacunas.

10.6. Examples. When $n=2$, then $\partial \alpha^{*}=0$ for all $x$ in $K(A, \vartheta)-W(A, \vartheta)$ so that the entire complement of $W(A, \vartheta)$ consists of Petrovsky lacunas. When $n=3$, then by $(6.26)$, 
$\partial \alpha^{*}=0$ if and only if $A^{*} \cap X^{*}$ is real. In particular, all the curved triangles in the figures $5 \mathrm{~b}$ except those of $5 \mathrm{~b} 8$ are Petrovsky lacunas. Theorem 6.27 provides other examples for $n$ odd $>3$. When $n$ is even and $\operatorname{Re} X \cap A$ is empty, $x$ belongs to a Petrovsky lacuna. This applies in particular to the case when $a=\Delta$ is the wave operator and $x$ is inside the future light-cone.

The interest of the Petrovsky condition (10.4) is that it is both necessary and sufficient at least when a certain stability is required. To begin with we shall prove this for low dimensions without using stability.

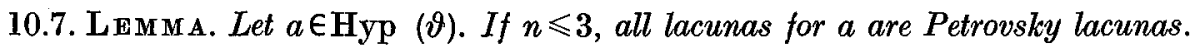

Proof. By Lemma 8.3, $W=W(A, \vartheta)$ is the singular support of $E(a, \vartheta, \cdot)$ so that all lacunas for $a$ are regular. The cases $n=1,2$ are trivial and the only thing we have to show is that, for $n=3, a$ complete and $x$ in a lacuna for $a$, we have $\partial \alpha(A, x, \vartheta)^{*}=0$, i.e., by (6.26), that $A^{*} \cap X^{*}$ is real. Since $a$ is complete, $m \geqslant 2$. Putting $|\nu|=m-2$ in $\left(7.17^{\prime}\right)$ and using figure $6 \mathrm{~b}$, if $E(a, \vartheta, \cdot)$ is a polynomial of degree $m-3$ close to $x$, we get $\int_{\gamma} Q(z) a_{x}(z)^{-1} d z=0$ for all polynomials $Q(z)$ of degree $\leqslant m-2$. Here $z$ is an inhomogeneous coordinate in $X^{*}$, $a_{x}$ is the restriction of $a$ to $X^{*}$ and $\gamma$ consists of small circles around the nonreal part of $A^{*} \cap X^{*}$ oriented by the sign of $\operatorname{Im} z$. If $w, \bar{w}$ are two points in this non-real part, a suitable choice of $Q$ gives $\int_{\gamma} d z /(z-w)(z-\bar{w}) \neq 0$ which is a contradiction.

A complete investigation of supports, singular supports and lacunas does not seem to be easy, but it may be fruitful to try to prove or disprove some simple and strong hypotheses which agree with known facts. We adopt the following very tentative

10.8. Conjeoture. Let $a \in \mathrm{Hyp}(\vartheta, m)$. Then

(i) all regular lacunas are Petrovsky lacunas.

(ii) $\operatorname{SS} E(a)=\bigcup \operatorname{SS} E\left(a_{\xi}\right)$ for $0 \neq \xi \in \operatorname{Re} Z$.

(iii) $E(a)$ is holomorphic outside $\operatorname{SSE}(a)$.

By Theorem 10.3 and Lemma 9.6, the first part of this conjecture implies the corresponding statement for non-homogeneous operators. In view of the structure of the Herglotz-Petrovsky-Leray formulas, it is tempting to consider also the statement

$$
m \geqslant n \Rightarrow \mathrm{S} E(a)=K(A),
$$

but, as we have seen in Example 8.7, this statement is wrong. It may still be true outside the product situation considered there.

Petrovsky proved a weak version of (i), namely that if $a \in \mathrm{Hyp}^{0}(\vartheta)$ and $A^{*}$ is regular then every stable lacuna is a Petrovsky lacuna. Petrovsky's method was to calculate 
$H_{n-2}\left(A^{*}\right)$ for general non-singular $A^{*}$ and to study Abelian integrals on $A^{*}$ as functions of $a$. In Part II of this paper we shall prove more precise results using theorems on the cohomology at algebraic varieties due to Atiyah and Hodge (1955) and generalized by Grothendieck (1966). We state some of them here. By Grothendieck's theorem, all rational forms on projective space $Z^{*}$ with poles on $A^{*} \cup X^{*}$ span the cohomology of $Z^{*}-A^{*} \cup X^{*}$. Now all such forms of degree $n-1$ appear in the formula $\left(7.17^{\prime}\right)$ for $D^{\nu} E\left(a^{k}, x\right)$ when $m k-n-|v|<0$ and $k=1,2, \ldots$ and it is easy to show that the kernel of the tube operation $t_{x}$ vanishes. Hence, any component of $\mathrm{CW}(A, \vartheta)$ which is a lacuna for all powers of $a$ is a Petrovsky lacuna. Note that a similar cohomology argument was used in a very simple case in the proof of Lemma 10.7. When $\partial \alpha(A, x, \vartheta)^{*}=0$, then $\alpha(A, x, \vartheta)^{*}$ comes from an element of $H_{n-1}\left(Z^{*}-A^{*}\right)$ and we can apply the same argument to (7.17) with $a$ replaced by powers of $a$. The conclusion is that $\alpha(A, x, \vartheta)^{*}=0$, but we know this to be true only when $x$ is outside $\pm K(A, \vartheta)^{*}$. Hence, there is no non-trivial strong lacuna common to all powers of $a$. In both cases, we can also use a sharper form of Grothendieck's theorem to be proved in Part II, namely that in order to span the desired cohomology groups, we need only forms with a bound on the order of the poles depending only on $m$ and $n$. What we then get is

10.9. THEOR स. Let $a \in H_{y p}(\vartheta, m)$. There are numbers $k_{0}$ and $k_{1}$ depending only on $m$ and $n$ such that,

(i) all regular lacunas for $a^{k}$ are Petrovsky lacunas when $k \geqslant k_{0}$.

(ii) The trivial lacuna is the only strong lacuna for $a^{k}$ when $k \geqslant k_{1}$.

In fact, (ii) follows from the above reasoning and also (i), restricted to regular lacunas. Now (ii) is precisely the statement that $\mathrm{S} E\left(a^{k}, \vartheta, \cdot\right)=K(A, \vartheta)$. It implies Theorem 8.9 so that, if $k$ is large enough, $\operatorname{SS}\left(a^{k}, \vartheta, \cdot\right)=W(A, \vartheta)$ and this means that $a^{k}$ has only regular lacunas.

10.10. Corollary. Let $a \in \mathrm{Hyp}(\vartheta)$ and let $L$ be a component of $K(A, \vartheta)-W(A, \vartheta)$. If the Petrovsky condition (10.4) holds for one $x$ in $L$, it holds for all $x$ in $L$.

Proof. Theorem 10.3, Theorem 10.9 (i) and the definition of a Petrovsky lacuma.

Example. In example $8.8, \dot{K}$, i.e. the set of all $x>0$, is a Petrovsky lacuna. That the Petrovsky condition (10.4) holds for such an $x$ is a non-trivial fact.

We shall also prove some more direct generalizations of Petrovsky's result that do not involve powers of $a$, but then we have to require some regularity of $A^{*}$ or stability under 
certain restricted perturbation families. In particular if $A^{*}$ is regular, all lacunas are Petrovsky lacunas and hence also stable under all hyperbolic perturbations. The other parts of the conjecture are also true in this case, and (iv) holds when $n \leqslant 3$, and may be true for all $n$. In any case, when $m \geqslant n, a$ has no stable strong lacunas inside $K(A)$.

\section{Remarks about systems}

Let $P \in \operatorname{hyp}_{r}(\vartheta)$, i.e. $P=P(D)$ has coefficients in the ring of complex $r \times r$ matrices and $\operatorname{det} P \in$ hyp $(\vartheta)$, and let $E=E(P)=E(P, \vartheta, \cdot)$ be the corresponding fundamental solution. By virtue of $(3.3), E(P)$ is locally holomorphic outside $W(A, \vartheta)$ where $a$ is the principal part of $\operatorname{det} P$. More generally, every component $E_{j k}$ of $E$ has the form

$$
E_{j k}=Q_{j k}(D) E\left(P_{j k}\right)
$$

where $Q_{j k}(D)$ is a polynomial, $P_{j k} \in$ hyp $(\vartheta)$ is a factor of $\operatorname{det} P$ and $P_{j k}$ and $Q_{j k}$ are relatively prime. Hence, if $a_{j k}$ is the principal part of $P_{j k}, E_{j k}(P)$ is locally holomorphic outside $W\left(A_{j k}, \vartheta\right) \subset W(A, \vartheta)$. The notions of sharp front, lacuna and strong lacuna extend immediately to $E$ or any $E_{j k}$ and whenever $E(a)$ or $E\left(a_{j k}\right)$ has such an object, $E$ and $E_{j k}$ respectively also have it. Also, if $P_{j k}=a_{j k}$ has homogeneity $m_{j k}$ and $Q_{j k}$ has degree> $m_{j k}-n$, a lacuna for $a_{j k}$ is a strong lacuna of $E_{j k}$. For this rather trivial reason, $E_{j k}$ or $E$ may have more strong lacunas than $E\left(a_{j k}\right)$ or $E(a)$ respectively.

Converse propositions of the type that lacunas for $P$ are also lacunas for $\operatorname{det} P$ should be deduced from more detailed ones, namely that lacunas of $E_{j k}$ are also lacunas for $P_{j k}$. Throwing away the indices we are lead to the following problem about scalar operators. Let $P \in$ hyp $(\vartheta)$ and let $Q(D)$ be a polynomial. When is a lacuna $L$ of $Q(D) E(P, \vartheta, \cdot)$ a lacuna of $E(P, \vartheta, \cdot)$ ? Taking $P=a$ homogeneous, it is easy to see that it suffices to suppose that also $Q$ is homogeneous. Requiring that $L$ be a Petrovsky lacuna for $a$ and using Theorem 7.16 , we have the following algebraic problem. Is it true that all rational $(n-1)$-forms on $Z^{*}-A^{*} \cup X^{*}$ with poles of order 1 on $A^{*}$ and arbitrary order on $X^{*}$ and divisible by a fixed polynomial $Q$ span $H_{n-1}\left(Z^{*}-A^{*} \cup X^{*}\right)$ ? As we shall see in Part II the answer is affirmative for instance when $A^{*}$ is non-singular and $Q \neq 0$ is relatively prime to $a$. When $n \leqslant 3$, a rather complete analysis is possible. Systems with $n=3$ possessing lacunas occur in magnetohydrodynamics and have been studied extensively by various authors (Weitzner (1961), Bazer and Yen (1967), (1969), Burridge (1967)).

\section{References}

Ativan, M. F.\&HodGe, W. D. V., Integrals of the second kind of an algebraic variety. Ann. of Math., 62 (1955), 56-91.

BAZER, J. \& YEN, D. H. Y., The Riemann matrix of $(2+1)$-dimensional symmetric-hyperbolic systems. Comm. Pure Appl. Math., 20 (1967), 329-363. 
- Lacunas of the Riemann matrix of symmetric-hyperbolic systems in two space variables. Preprint. Courant Institute, Now York University, 1969.

Borovikov, V. A., The elementary solution of partial differential equations with constant coefficients. Trudy Moskov. Mat. Obšč., 66, 8 (1959), 159-257

Some sufficient conditions for the absence of lacunas. Mat. Sb., 55 (97) (1961), 237-254.

Burridae, R., Lacunas in two-dimensional wave propagation. Proc. Cambridge Phil. Soc., 63 (1967), 819-825.

GÅRDING, L., The solution of Cauchy's problem for two totally hyperbolic linear differential equations by means of Riesz integrals. Ann. of Math., 48 (1947), 785-826. Errata ibid. 52 (1950), 506-507.

Linear hyperbolic partial differential equations with constant coefficients. Acta Math., 85 (1950), 1-62.

An inequality for hyperbolic polynomials. J. Math. Mech., 8 (1959), 957-966.

- Transformation de Fourier des distributions homogènes. Bull. Soc. math. France, 89 (1961), $381-428$.

The theory of lacunas. Battelle Seattle 1968 Recontres. Springer (1969).

Gelfand, I. M. \& Shrlov, G. E., Generalized functions I. Moscow 1958.

Gindikin, S. G., Cauchy's problem for strongly homogeneous differential operators. Trudy Moslov. Mat. Obš̌̌., 16 (1967), 181-208.

Gindikin, S. G. \& VAJNBERG, B.R., On a strong form of Huygens' principle for a class of differential operators with constant coefficients. Trudy Moskov. Mat. Obšc., 16 (1967), 151-180.

GrothendIEck, A., On the de Rham cohomology of algebraic varities. Publ. IHES, 29 (1966), $351-359$.

Hadamard, J., Le problème de Cauchy et les équations aux dérivées partielles linéaires hyperboliques. Paris 1932.

Herglotz, G., Über die Integration linearer partieller Differentialgleichungen I. (Anwendung Abelscher Integrale) II, III (Anwendung Fourierscher Integrale). Leipzig. Ber. Sächs. Akad. Wiss., Math Phys. Kl., 78 (1926), 93-126; 80 (1928), 6-114.

Hörmander, L., Linear partial differential operators. Springer 1963.

- On the singularities of solutions of partial differential equations. International Conference of Functional Analysis and Related Topics. Tokyo 1969.

Leray, J., Un prolongement de la transformation de Laplace ... (Problème de Cauchy IV). Bull. Soc. math. France, 90 (1962), 39-156.

- Hyperbolic differential equations. The Institute for Advanced Study, Princeton N. J. (1952).

Ludwig, D., Singularities of superpositions of distributions. Pacific J. Math. 15 (1965), $215-239$.

NuiJ, W., A note on hyperbolic polynomials. Math. Scand., 23 (1968), 69-72.

Petrovsky, I. G., On the diffusion of waves and the lacunas for hyperbolic equations. Mat. $S b$., 17 (59) (1945), 289-370.

Riesz, M., L'intégrale de Riemann-Liouville et le problème de Cauchy. Acta Math., 81 (1949), 1-223.

Schwartz, L., Théorie des distributions I, II. Paris (1950-51).

SteltmaCher, K. L., Eine Klasse huygenscher Differentialgleichungen und ihre Integration. Math. Ann., 130 (1955), 219-233.

Svensson, L., Necessary and sufficient conditions for the hyperbolicity of polynomials with hyperbolic principal part. To be published in Ark. Mat., 8 (1970).

Weitzner, H., Green's function for two-dimensional magnetohydrodynamic waves I, II. Phys. Fluids, 4 (1961), 1250-1258. 